\title{
Sparse Tensor Transpositions
}

\author{
Suzanne Mueller \\ MIT CSAIL \\ suzmue@csail.mit.edu
}

\author{
Peter Ahrens \\ MIT CSAIL \\ pahrens@csail.mit.edu
}

\author{
Stephen Chou \\ MIT CSAIL \\ s3chou@csail.mit.edu
}

\author{
Fredrik Kjolstad \\ Stanford University \\ kjolstad@stanford.edu
}

\author{
Saman Amarasinghe \\ MIT CSAIL \\ saman@csail.mit.edu
}

\begin{abstract}
We present a new algorithm for transposing sparse tensors called Quesadilla. The algorithm converts the sparse tensor data structure to a list of coordinates and sorts it with a fast multi-pass radix algorithm that exploits knowledge of the requested transposition and the tensors input partial coordinate ordering to provably minimize the number of parallel partial sorting passes. We evaluate both a serial and a parallel implementation of QuESADILLA on a set of 19 tensors from the FROSTT collection, a set of tensors taken from scientific and data analytic applications. We compare QuesAdilla and a generalization, Top-2-SADILLA to several state of the art approaches, including the tensor transposition routine used in the SPLATT tensor factorization library. In serial tests, QUESADILLA was the best strategy for $60 \%$ of all tensor and transposition combinations and improved over SPLATT by at least $19 \%$ in half of the combinations. In parallel tests, at least one of QUESADILLA or Top-2-SADILLA was the best strategy for $52 \%$ of all tensor and transposition combinations.
\end{abstract}

\section{CCS CONCEPTS}

- Mathematics of computing $\rightarrow$ Mathematical software performance; - Theory of computation $\rightarrow$ Sorting and searching; • Software and its engineering $\rightarrow$ Source code generation.

\section{KEYWORDS}

Sparse Tensors, Transposition, Sorting, COO, Radix Sort

\section{INTRODUCTION}

Tensors generalize vectors and matrices to any number of dimensions. Tensors used in computation are often sparse, which means many of the values are zero. To take advantage of the large number of zeroes in the tensor, we use sparse formats that allow the zeroes to be compressed away. These formats range from a simple list of coordinates to complicated data structures such as Compressed Sparse Row (CSR) [8], Doubly Compressed Sparse Row (DCSR) [4], Block Compressed Sparse Row (BCSR) [13], and Compressed Sparse Fiber (CSF) [23]. These formats have a natural ordering of their dimensions that provides a lexicographical ordering of the tensor nonzeros. In a sorted list of coordinates, the order of the sorting keys determines this lexicographic ordering.

Tensor algebra is used to compute with data stored in tensors. These multidimensional computations need to access the nonzero entries in one or more tensors, compute, and store the results. Accessing the nonzero entries requires some traversal of the tensor. However, unlike for dense tensors, traversing the nonzeros of a sparse tensor in different lexicographical orderings may be asymptotically more expensive than the natural lexicographical ordering. Therefore, it is often faster to first transpose input tensors by reordering their dimensions before executing tensor expressions. This way, the tensor can be accessed naturally in the expression itself.

Tensor transposition is ubiquitous in data processing. Anytime multiple tensor expressions are composed and the output of one expression must be used as an input to the next, with a different index ordering and possibly a different sparse format, we need to transpose. For example, element-wise operations between tensors without matching index orderings (thus requiring transposition as a bottleneck) is listed as one of the five benchmark operations in the Parallel Sparse Tensor Algorithm Benchmark Suite (PASTA) [17]. Sparse tensor transposition may also occur when several different orderings of input data are required for efficient operation, but the space is not available to hold all of them. Such a situation might arise when using an alternating least squares method for canonical polyadic decomposition [23].

Prior work has focused extensively on dense tensor transpositions [6, 11, 14, 15, 20, 24-27]; we refer readers to [24] for a summary. Sparse matrix and tensor transposition have received relatively little attention [29]. A fast CSR sparse matrix transposition algorithm is proposed in [12], and improvements are proposed in [9, 29]. Further variations on sparse matrix transposition are discussed in $[5,10,28,30,31]$. None of these techniques, however, readily generalize to sparse tensor transposition with input tensors of arbitrary ranks.

Tensors are often stored as a list of coordinates of nonzeros. If the coordinates are ordered lexicographically, adjacent coordinates may share the same indices in the first several modes. The Compressed Sparse Fiber (CSF) format [23] compresses these duplicate nonzeros using a tree-like storage format. In CSF, nodes represent indices, leaves represent nonzeros and paths from root to leaf represent coordinates. The children of each node are ordered. The matrix case of CSF is called Compressed Sparse Row (CSR).

More complicated sparse tensor formats like CSF often have similar ordering constraints, and require access to the coordinates in some lexicographic order in order to construct the tensor. The current state of the art for transposing sparse tensors involves converting the sparse tensor into a list of coordinates, sorting the list of coordinates, and finally packing the list of coordinates into the desired sparse tensor format [23].

This approach reduces the problem of transposing a tensor into a problem of sorting a list of coordinates. However, the lists of coordinates have partial orderings we can use to accelerate the sorting algorithms. Consider the example matrix in Figure 1. In 
order to transpose the matrix, the column coordinates must be ordered lexicographically before the row coordinates. This could be accomplished by sorting with the column coordinate as the primary key and the row coordinate as the secondary key.

We can do better than that. The coordinates are already sorted on the row coordinates. By doing a stable sort on just the column coordinate, we get the same result. In this paper, we will generalize this optimization to arbitrary tensor transpositions.
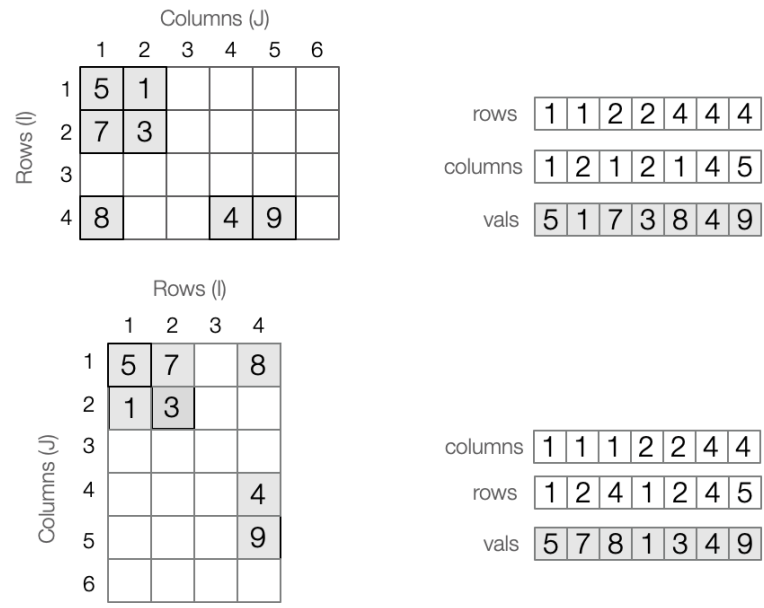

$$
\begin{aligned}
& \text { columns } \begin{array}{l|l|l|l|l|l|l|}
\hline 1 & 1 & 1 & 2 & 2 & 4 & 4 \\
\hline
\end{array} \\
& \text { rows } \begin{array}{l|l|l|l|l|l|l|l|}
\hline 1 & 2 & 4 & 1 & 2 & 4 & 5 \\
\hline
\end{array} \\
& \text { vals } \begin{array}{ll|l|l|l|l|l|l|}
5 & 7 & 8 & 1 & 3 & 4 & 9
\end{array}
\end{aligned}
$$

Figure 1: The matrix $A$ can be represented as a list of coordinates including only the nonzero values. Transposing the tensor in this format switches the lexicographic ordering of the rows and columns, such that the columns appear first. The top list of coordinates represent the matrix in the top left. The bottom list of coordinates represent the transposed version of this matrix.

The main contributions of this work are:

(1) A decomposition of tensor transposition into parallelizeable near-linear-work partial sorts (one of the two partial sorts is novel) that optionally respect previous partial orderings.

(2) An algorithm that uses partial orderings in the original sparse tensor format to minimize the number of partial sorts required by the transposition algorithm. This relates the parallel span of radix sorting to partial orderings in the input.

(3) A parallel implementation that demonstrates this transposition algorithm is competitive with, and often faster than, state of the art approaches.

\section{BACKGROUND}

A tensor of rank $r$ is a multidimensional array that associates $r$ tuples (referred to as coordinates) with values, or entries. We refer to the $k^{t h}$ position in a coordinate as mode $k$. The size of a tensor is specified by an $r$-tuple of dimensions $n$, where each index $i_{k}$ is an integer in the range $1 \leq i_{k} \leq n_{k}$.

Let $N$ be the number of nonzero entries in our tensor. A tensor is sparse if most of its entries are zero. This has led to the development of sparse tensor storage formats that support efficient computation over only the nonzero entries. These formats range from a simple sorted list of nonzero coordinates together with their values, the Coordinates (COO) format [2], to more complicated hierarchical mode-by-mode compression schemes such as Compressed Sparse Row (CSR) or Compressed Sparse Fiber (CSF) [8, 23]. All three of these formats induce a natural lexicographic ordering of the dimensions; iterating over the tensor in the natural order can be done very efficiently.

We define lexicographic ordering on $r$-tuples recursively using a tuple $\sigma$ of modes in order of their priority. We consider the coordinate $i=\left(i_{1}, i_{2}, \ldots\right)$ to be less than the coordinate $i^{\prime}=\left(i_{1}^{\prime}, i_{2}^{\prime}, \ldots\right)$ under the ordering $\sigma$ in two cases. The first case is when $i_{\sigma_{1}}<i_{\sigma_{1}}^{\prime}$. The second case is when both $i_{\sigma_{1}}=i_{\sigma_{1}}^{\prime}$ and $i<i^{\prime}$ under the ordering $\left(\sigma_{2}, \sigma_{3}, \ldots\right)$. For completeness, we say that all tuples are considered equal under $\sigma=()$. We will refer to the $(1,2, \ldots, r)$ ordering of $k$-tuples as the simple ordering. We say an ordering is complete if it contains $r$ distinct modes.

\subsection{Coordinates (COO)}

$\mathrm{COO}$ stores the nonzero coordinates in the tensor as a list of $\sigma$ sorted coordinates. Transposing a tensor in $\mathrm{COO}$ format is equivalent to reordering the coordinate list to a new complete ordering. This simplicity makes COO a popular format; it is the only sparse tensor format for the MATLAB Tensor Toolbox and TensorFlow libraries, and is used as an intermediate format during transpositions in the SPLATT library [1, 2, 23]. Since most sparse tensor formats can be converted to and from COO format and the format is readily sorted, we focus on transposing tensors in $\mathrm{COO}$ format.

The COO format can be implemented either with a list of lists (one for each mode) or as a list of coordinate tuples. We will use the latter for notational purposes. Thus, we store an array $A$ in COO using two arrays, A.crd and A.val. The $c r d$ array is an array of coordinates, and val is an array of corresponding values. This requires $O(r)$ bits to store each coordinate, so the total storage requirement for indices is $O(r * N)$ bits. Figure 2 shows an example of $\mathrm{COO}$ storage.

\subsection{Transposition}

The COO, CSR, and CSF formats are all sorted by lexicographic orderings on the coordinates. In $\mathrm{COO}$, we sort the coordinates by some $\sigma$ ordering, and in CSR and CSF, $\sigma$ gives the order in which modes correspond to levels in the tree. CSR and CSF formats sort each level by the indices within that mode. In this work, transposition corresponds to a change in this storage order.

We will express tensor transposition operations using the final desired storage order $\sigma$. Without loss of generality, we assume the tensor is initially ordered by $(1,2, \ldots)$. For example, transposing a matrix stored in $(1,2)$ order is equivalent to changing the storage order to $\sigma=(2,1)$, then relabeling the modes.

Certain computations will perform better when the dimensions can be iterated over efficiently in a different order than the initial storage order $[16,23]$. When we encounter such a computation, it will be beneficial to transpose the tensor.

Tensors can be reordered using any sorting method. Since the coordinates come from a fixed range of values, we can also use sorts that work on fixed length keys, like histogram or radix sorts [7]. SPLATT, a sparse tensor library designed to be highly parallel, 


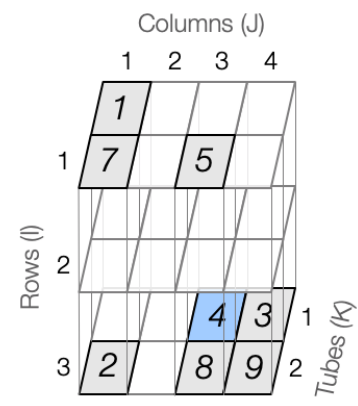

$$
\begin{array}{c|c|c|c|c|c|c|c|c|}
\text { rows } & 1 & 1 & 1 & 3 & 3 & 3 & 3 & 3 \\
\hline \text { columns } 1 & 1 & 1 & 3 & 1 & 3 & 3 & 4 & 4 \\
\hline \text { tubes } 1 & 1 & 2 & 2 & 2 & 1 & 2 & 1 & 2 \\
\text { vals } 1 & 1 & 7 & 5 & 2 & 4 & 8 & 3 & 9 \\
\hline
\end{array}
$$

Figure 2: $\mathrm{COO}$ represents a tensor as a list of coordinates with all of the zero values compressed out. The list of arrays in the bottom left represent the tensor in the top left.

uses a specialized sorting strategy to take advantage of potential parallelism that exists in the problem [23]. SPLATT chooses to first do a histogram sort on mode $\sigma_{1}$. It then sorts the coordinates for each index in mode $i_{\sigma_{1}}$ using $n_{\sigma_{1}}$ separate calls to quicksort. In the sequential implementation this strategy benefits from smaller subproblems for quicksort. In the parallel version, SPLATT is able to sort these buckets in parallel.

\section{ALGORITHMS}

A naive algorithm for sparse tensor transposition is to comparison sort the coordinates into the desired lexicographic order. However, since coordinates already have an initial ordering, we can think of sorting coordinates as simply changing the lexicographic ordering to prioritize different dimensions. It takes $O(r)$ time to compare two coordinates of an $r$-tensor. Thus, a comparison based coordinate sort would run in $O(r N \log N)$ time. However, since the indices are bounded by the dimensions, we can use parallelizable stable sorts like a histogram sort (called counting sort in $[7,19])$ to sort the coordinates on a single mode $k$ in $O\left(r N+n_{k}\right)$ time. If we perform $r$ histogram sorts (a radix sort on $r$-digit numbers), we can sort our coordinate list in $O\left(r^{2} N+n_{1}+n_{2}+\cdots\right)$ time, an asymptotic improvement over comparison sort when the dimensions are small. If we assume that coordinates are each processed in constant time, our histogram sort takes $O\left(N+n_{k}\right)$ time and our radix sort takes $O\left(r N+n_{1}+n_{2}+\cdots\right)$ time. This algorithm can be improved further; for some transpositions, we do not need to perform all $r$ sorts. For example, HALFPERM uses a histogram sort to prioritize the second dimension in the new ordering. Depending on the size of the second dimension, this single histogram sort is faster than a generic sort of the coordinates, and certainly faster than redundantly sorting the first dimension before sorting the second.
In this section, we formalize and generalize this idea to produce the Quesadilla tensor transposition algorithm, which provably performs the minimal number of histogram sorts. We start with a description of our histogram partial sort and a bucketing modification to produce two sorting primitives. We then use these primitives to build the Quesadilla and Top- $K$-sadilla tensor transposition algorithms.

\subsection{Histogram Partial Sorts}

A histogram sort sorts integer keys of bounded size. It first counts the number of occurrences of each key values. It then performs a prefix sum, also known as a cumulative sum or prefix scan, over the array of counts to determine where each group of equivalent keys will lie in the output array. This reserves enough space for all of the coordinates to appear in the output order, and the scanned array can be used record how full each output group is as the algorithm puts each coordinate directly into its output location. There is extensive research on the topic of parallelizing histogram sort as a subroutine of radix sort [19]. In our experiments, we use the same implementation as SPLATT [23], where each processor uses a private copy of count which is synchronized before moving coordinates to their output destinations.

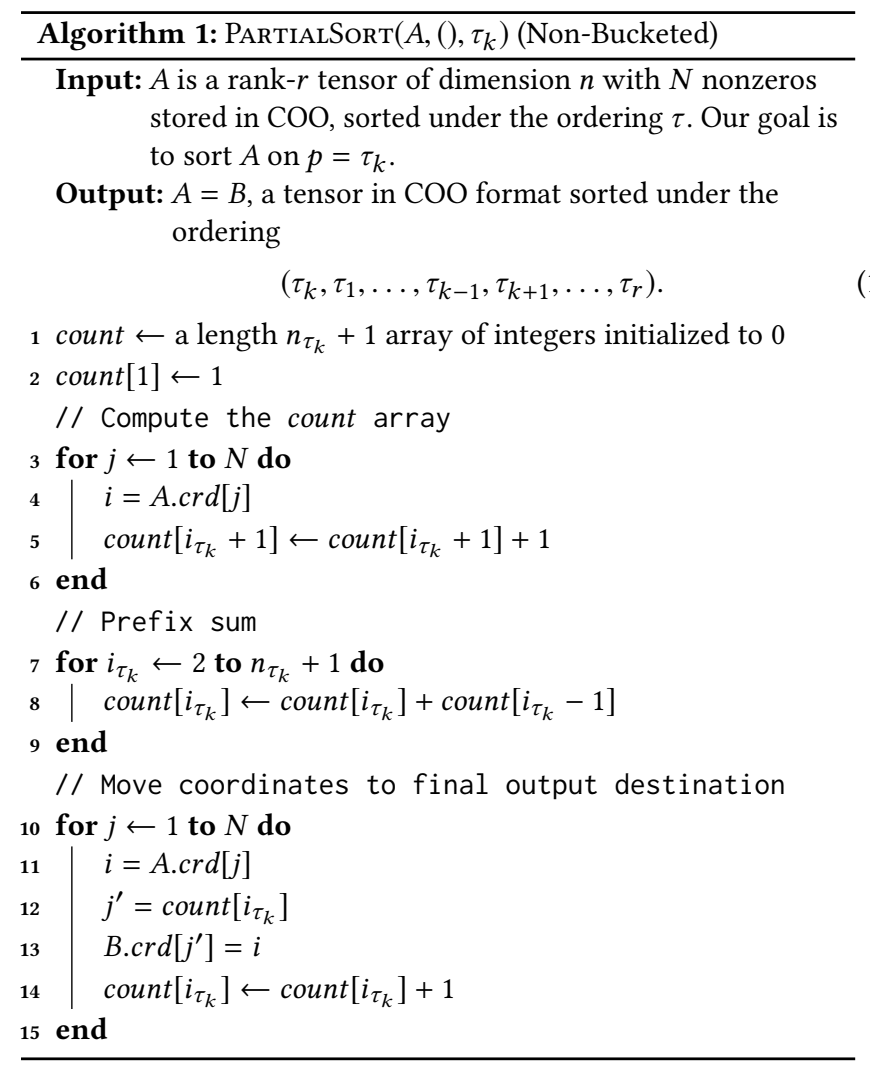

The histogram sort iterates over the coordinates twice and the count array once. The total runtime is $O\left(r N+n_{\tau_{k}}\right)$, where $n_{\tau_{k}}$ is the dimension of the mode being sorted on. If we can process coordinates in constant time, the runtime is $O\left(N+n_{\tau_{k}}\right)$. The histogram sort clearly produces a lexicographic ordering which prioritizes $\tau_{k}$ 
first. Since the sort is stable, the relative ordering of other modes is unaffected. Thus, it moves the mode $\tau_{k}$ to be the first mode in the lexicographic order, as described in (1).

\subsection{Bucketed Histogram Sort}

Although radix sort is most commonly performed from the least significant digit to the most significant digit, it will be useful for us to be able to work backwards sometimes, sorting one mode while respecting another partial ordering. Informally, we wish to sort a mode to a different position than the first spot in the output ordering. Formally, if our tensor is sorted with respect to $\tau$, we wish to sort on $\tau_{k}$ while leaving the ordering $\left(\tau_{1}, \ldots, \tau_{l}\right)$ of the first $l<k$ modes unaffected. This means that we need to sort each group of contiguous coordinates (a bucket) which agree on the values $i_{\tau_{1}}, \ldots, i_{\tau_{l}}$. If we use a comparison-based sort within each bucket, we can perform the sort recursively but incur a logarithmic overhead. If we use a radix-based algorithm within each bucket, we need to perform an $O\left(n_{\tau_{k}}\right)$ prefix sum in each bucket. Since the number of buckets is bounded only by $N$, the resulting runtime of $O\left(r N n_{\tau_{k}}\right)$ is unacceptable.

Here, we describe a variation on histogram sort that discovers the buckets for $\left(\tau_{1}, \ldots, \tau_{l}\right)$, sorts on $\tau_{k}$, then reimposes the previous ordering. Since there are at most $N$ buckets, our algorithm runs in time $O\left(N *(l+1)+n_{\tau_{k}}\right)$. If we assume operations on coordinates occur in constant time, our algorithm runs in time $O\left(N+n_{\tau_{k}}\right)$. Note that the input must be sorted under $\left(\tau_{1}, \ldots, \tau_{l}\right)$ to discover the buckets by examining adjacent coordinates.

Algorithms 1 and 2 are both called PARTIALSort; we use Algorithm 1 when $l=0$ and Algorithm 2 otherwise.

Although we can save buckets as we fill the count array, Algorithm 2 performs an extra bucketing step to create the perm array, and the perm array introduces more indirection in the final bucketing step than the similar loop in Algorithm 1. Saving the buckets takes $O(l N)$ time, the prefix sum takes $O\left(n_{\tau_{k}}\right)$ time, and the last two bucketing steps take $O(r N)$ time. The total runtime of bucketed histogram sort is $O\left(r N+n_{\tau_{k}}\right)$, or $O\left(N+n_{\tau_{k}}\right)$ if we assume constant-time operations on coordinates.

Bucketed histogram sort works by first stably sorting on mode $\tau_{k}$, then by sorting on $\left(\tau_{1}, \ldots, \tau_{l}\right)$ using the bucket array. After the loop on line 24, perm sorts $A$ under (2)

$$
\left(\tau_{k}, \tau_{1}, \ldots, \tau_{k-1}, \tau_{k+1}, \ldots, \tau_{r}\right)
$$

Since the stored buckets correspond to equivalence classes of $\left(\tau_{1}, \ldots, \tau_{l}\right)$ in order, the loop on line 29 sorts $A$ stably on the buckets, reprioritizing $\left(\tau_{1}, \ldots, \tau_{l}\right)$ in the ordering to produce the final order

$$
\left(\tau_{1}, \ldots, \tau_{l}, \tau_{k}, \tau_{l+1}, \ldots, \tau_{k-1}, \tau_{k+1}, \ldots, \tau_{r}\right) .
$$

As we describe parallelization strategies, we focus our attention on these three steps. Algorithm 2 discovers the buckets, stably sorts on the desired mode, then stably sorts on the buckets. Discovering the buckets is a simple linear-time algorithm that we can easily parallelize, taking care to account for buckets that cross processor boundaries. Most parallel implementations of histogram sort, including SPLATT, create private copies of the count array [23]. On $P$ processors, these implementations run in $O\left(N / P+n_{\tau_{k}}\right)$ time. Since we can usually assume the dimension of the mode to be sorted is

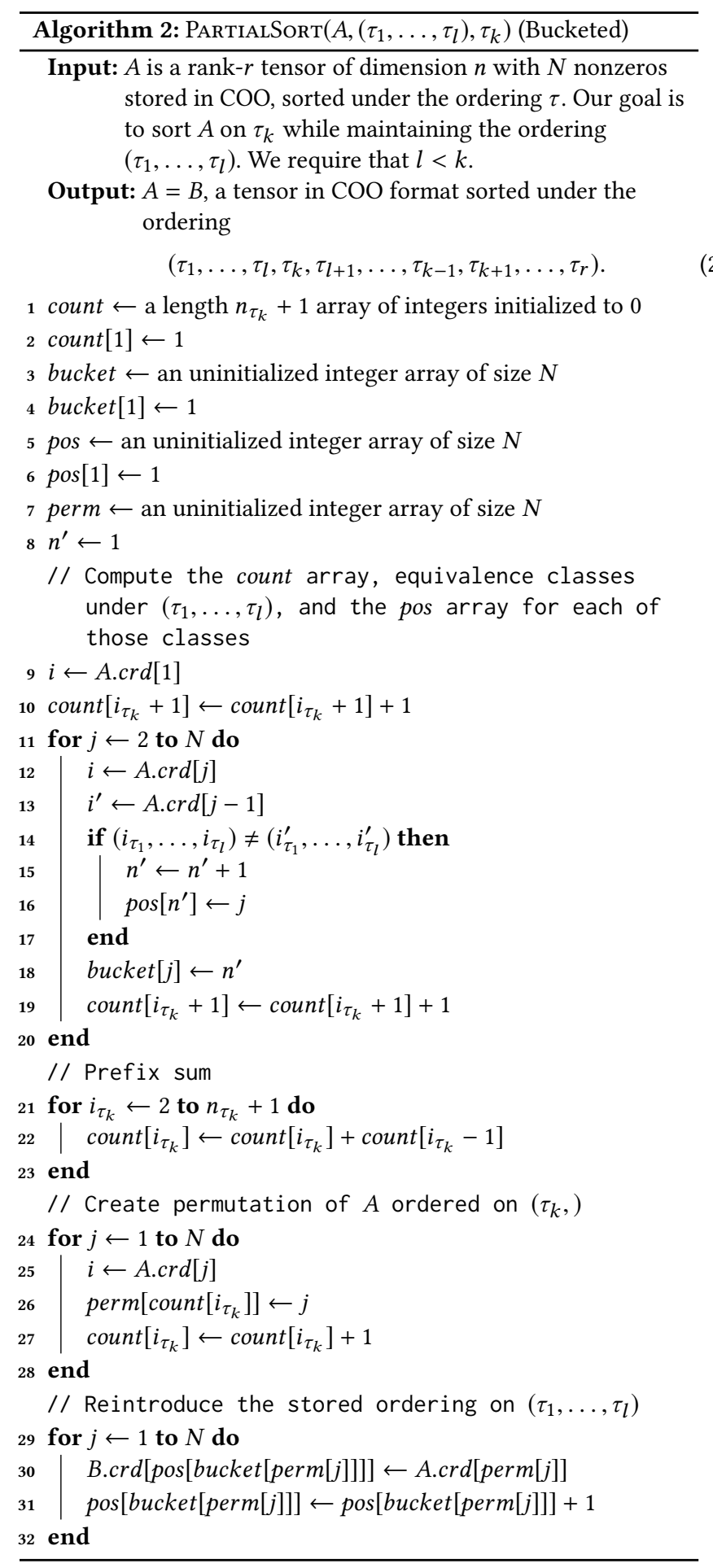

small relative to the number of nonzeros, these parallel implementations of histogram sort are acceptable for sorting the desired mode. However, we cannot assume that the number of buckets is small relative to the number of nonzeros. To effectively parallelize the second sort, we would need to use an algorithm whose runtime is 
linear in both the number of nonzeros and the range of keys to be sorted, such as a sample sort $[3,33]$. Notice that the sampling step can be avoided because the bucket discovery step calculates the exact distribution of buckets (keys).

We can simplify parallelization of Algorithm 2 by decomposing the problem along bucket boundaries. The buckets limit the travel of coordinates between input and output orderings; coordinates do not escape their buckets. Therefore, running Algorithm 2 on a contiguous region of input buckets will compute the corresponding region of the output ordering. This gives our chosen parallel algorithm where we assign to each processor the buckets which begin in their region, and each processor simply runs Algorithm 2 locally on their section. Assuming that the buckets are small enough to permit effective decomposition, this algorithm also runs in time $O\left(N / P+n_{\tau_{k}}\right)$. Notice that because SPLATT decomposes the local sorts along the index $\sigma_{1}$, SPLATT operates under the similar assumption that slices of the tensor are small enough to permit effective decomposition.

\subsection{Bucketed Histogram Sort Example}

We give an example of our bucketed histogram sort on a 4-tensor. For simplicity of presentation, we represent our coordinate list as 4-digit integers. The integers are initially sorted under the ordering $(1,2,3,4)$.

A.crd $=[1218,1224,1274,1421,1437,1456,1472,3216,3283,3286]$

Suppose that we would like them to be sorted under the ordering $\sigma=(1,2,4,3)$. We rearrange our digits to show the current ordering.

A.crd $_{\sigma}=[1281,1242,1247,1412,1473,1465,1427,3261,3238,3268]$

Since our ordering doesn't change the first two digits, we can re$\operatorname{order} A$ to be sorted under $\sigma$ by bucketing on the first two digits. Our algorithm starts by discovering the buckets and computing the bucket and pos arrays, which store the numbers and positions of each bucket:

$$
\begin{aligned}
& \text { A.crd } \sigma=[\underbrace{1412,1473,1465,1427}_{\text {“12..." } 1281,1242,1247}, \underbrace{14261,3238,3268}_{\text {"14..." }} \\
& \text { bucket }=[1,1,1,2,2,2,2,3,3,3] \\
& \text { pos }=[1,4,8, \text { undefined }, \ldots] \\
& \text { perm }=[4,7,9,2,3,6,8,10,5,1] \\
& \text { A.crd }[\text { perm }]= \\
& \text { [1421, 1472, 3283, 1224, 1274, 1456, 3216, 3286, 1437, 1218] } \\
& \text { A.crd } \sigma[\text { perm }]= \\
& \text { [1412, 1427, 3238, 1242, 1247, 1465, 3261, 3268, 1473, 1281] }
\end{aligned}
$$

At this point, if we restrict our attention to one bucket at a time, the coordinates are sorted. We just need to put each element of $A[$ perm $]$ back into it's corresponding bucket by sorting on bucket $[$ perm $]$. The pos array functions as the count array does in counting sort.

$$
\begin{aligned}
\text { B.crd } & =[1224,1274,1218,1421,1472,1456,1437, \\
B . c r d & =[\underbrace{1242,1243,3216,3286}_{\text {"12..." }}]
\end{aligned}
$$

Notice that B.crd $\sigma$ is lexicographically ordered, as desired.

\subsection{Minimizing Partial Sorts}

Transposition via a full radix sort would consist of $r$ calls to Algorithm 1. Not all transpositions, however, are equally difficult. For example, if we have a simply ordered 4-tensor and are asked to transpose it to the ordering $(4,1,2,3)$, this can be accomplished with the single call PartialSort $(A,(), 4)$, as seen in (1). On the other hand, if we are asked to transpose to $(4,3,2,1)$, we show that this requires at least 3 calls PARTIALSORT, since the only relevant partial ordering we can use is that of the first mode. In this work, we generalize this insight to produce the QUESADILLA algorithm which transposes tensors to a given target ordering with the minimal number of calls to either Algorithm 1 or 2 .

Although Algorithms 1 and 2 perform similar tasks, Algorithm 2 streams through and randomly accesses more vectors than Algorithm 1 does. If we count the number of unique vectors in each loop body separately (including initialization), Algorithm 1 streams through 4 vectors and randomly accesses 4 vectors, while Algorithm 2 streams through 7 vectors, and randomly accesses 7 vectors. While the costs of these algorithms are similar, they are not identical, and we should prefer to avoid the bucketed histogram variant whenever possible. For example, we can transpose to $(2,4,1,3)$ by calling $\operatorname{PartialSort}(A,(), 2)$ and then $\operatorname{Partial} \operatorname{Sort}(A,(2), 4)$, but we can avoid a bucketed histogram sort by calling PartialSort $(A,(), 4)$ and then PartialSort $(A,(), 2)$. Among transpositions that use the minimum number of partial sorts, we show that QuEsAdilla uses the minimal number of bucketed partial sorts (Algorithm 2). Thus, our algorithm minimizes a cost model that weighs each pass equally, but breaks ties towards the non-bucketed variant.

We start by showing that for a given target order $\sigma$, we must sort on a certain set of modes and that in order to achieve the minimum number of sorts, some of these sorts must be bucketed. We then give an algorithm that only sorts on this necessary set of modes, and only uses bucketed sorts when required.

3.4.1 Necessary Sorts. The number of dimensions $r$ is an upper bound on the number of passes needed to sort coordinates. This is the number of passes that are needed if we have a completely unsorted coordinate list and do a standard radix sort. The histogram sort and bucketed histogram sort can only move dimensions to the beginning of the lexicographic ordering. We use this fact to show a lower bound on the number of passes needed to sort the coordinates into the new lexicographic ordering. In several proofs, we will use a function $f(\tau, p)$ that we define on complete $r$-orderings $\tau$ as the set $\left\{\tau_{k+1}, \ldots, \tau_{r}\right\}$ where $\tau_{k}=p$. Thus, $f(\tau, p)$ is the set of modes which follow $p$ in the ordering $\tau$. For example, $f((1,3,2,4), 3)=\{2,4\}$.

Lemma 3.1. Let $A$ be a list of $r$-coordinates ordered by the complete ordering $\tau$. Assume that $A^{\prime}$ is the $\tau^{\prime}$ ordered result of calling $\operatorname{Partial} \operatorname{Sort}\left(A,\left(\tau_{1}, \ldots, \tau_{l}\right), p\right)$ where $p=\tau_{k}$ and $k>$ l. If $q \neq p$, then $f\left(\tau^{\prime}, q\right) \subseteq f(\tau, q)$.

Proof. The result follows from a close examination of (1) and (2) which describe the output ordering of Algorithms 1 and 2. Let $h$ be such that $\tau_{h}=q$. Note that $k>l$. If $1 \leq h \leq l$ or $k<h \leq r$, then $f\left(\tau^{\prime}, q\right)=f(\tau, q)$. Otherwise, $l<h<k$ and $f\left(\tau^{\prime}, q\right)=f(\tau, q) \backslash p \subset$ $f(\tau, q)$. 
This idea that the set following some mode never expands when we sort on a different mode allows us to show that certain modes must be direct arguments to PARTIALSORT at some point in our sequence of calls that transposes the tensor.

Lemma 3.2. Let $A$ be a list of $r$-coordinates ordered by the complete ordering $\tau$. Assume we wish to call PARTIALSORT some number of times to produce a $\sigma$ ordering of $A$, and that $f\left(\sigma, \sigma_{i}\right) \nsubseteq f\left(\tau, \sigma_{i}\right)$. Consider any sequence of statements of the form

$$
A \leftarrow \operatorname{Partial} \operatorname{Sort}\left(A,\left(\psi_{1}, \ldots, \psi_{l}\right), \psi_{k}\right),
$$

where $\psi$ is a complete intermediate ordering of $A, \psi_{k} \neq \sigma_{i}$, and $k>l$. No such sequence will result in a $\sigma$ ordering of $A$.

Therefore, any sequence of calls to SoRT designed to return a $\sigma$ ordering of $A$ must include a call for each value of $\sigma_{i}$ for which $f\left(\sigma, \sigma_{i}\right) \nsubseteq f\left(\tau, \sigma_{i}\right)$.

Proof. At some point in our sequence of calls, assume that $f\left(\sigma, \sigma_{i}\right) \nsubseteq f\left(\psi, \sigma_{i}\right)$, and let $\psi^{\prime}$ be the ordering after the next call to PARTIALSort. Lemma 3.1 implies that $f\left(\psi^{\prime}, \sigma_{i}\right) \subseteq f\left(\psi, \sigma_{i}\right)$, so it must still be the case that $f\left(\sigma, \sigma_{i}\right) \nsubseteq f\left(\psi^{\prime}, \sigma_{i}\right)$. Since we start with $f\left(\sigma, \sigma_{i}\right) \nsubseteq f\left(\tau, \sigma_{i}\right)$, there is no ordering in our sequence for which $f\left(\sigma, \sigma_{i}\right) \nsubseteq f\left(\psi, \sigma_{i}\right)$, and thus $\psi$ can never equal $\sigma$.

Lemma 3.2 implies a lower bound on the number of calls to PARTIALSORT required to transpose a $\tau$-ordered tensor $A$ to $\sigma$-order. We refer to this number with the function $b(\tau, \sigma)$. We define $b$ formally as the number of modes $i$ for which $f\left(\sigma, \sigma_{i}\right) \nsubseteq f\left(\tau, \sigma_{i}\right)$. For example, $b((1,3,2),(3,1,2))=1$. While $b$ gives us a lower bound on the number of sorts, it does not show a bound on whether each sort must be bucketed or not. We now show that no sequence of calls to PARTIALSORT of length $b(\tau, \sigma)$ may include a call $\operatorname{Partial} \operatorname{Sort}(A,(), p)$ if there exists $i$ such that $f\left(\sigma, \sigma_{i}\right) \subseteq f\left(\tau, \sigma_{i}\right)$ and $p \in f\left(\sigma, \sigma_{i}\right)$.

LeMma 3.3. Let $A$ be a list of $r$-coordinates ordered by the complete ordering $\tau$. Consider any length $b(\tau, \sigma)$ sequence of statements of the form

$$
A \leftarrow \operatorname{Partial} \operatorname{Sort}\left(A,\left(\psi_{1}, \ldots, \psi_{l}\right), \psi_{k}\right),
$$

where $\psi$ is a complete intermediate ordering of $A$ and $k>l$. If this sequence reaches the ordering $\sigma$, it may not contain any call where $l=$ 0 and there exists $i$ such that $f\left(\sigma, \sigma_{i}\right) \subseteq f\left(\tau, \sigma_{i}\right)$ and $\psi_{k} \in f\left(\sigma, \sigma_{i}\right)$.

Proof. Lemma 3.2 implies that we must sort on each mode where $f\left(\sigma, \sigma_{k}\right) \nsubseteq f\left(\tau, \sigma_{k}\right)$. Since our sequence only involves $b(\tau, \sigma)$ calls to PARTIALSORT, this sequence must only sort on these modes.

Assume for contradiction that our sequence involves a call where $l=0$ and there exists $i$ such that $f\left(\sigma, \sigma_{i}\right) \subseteq f\left(\tau, \sigma_{i}\right)$ and $\psi_{k} \in$ $f\left(\sigma, \sigma_{i}\right)$. Let $\psi^{\prime}$ be the ordering following $\psi$ after this call. Using (1), we see that $f\left(\sigma, \sigma_{i}\right) \nsubseteq f\left(\psi^{\prime}, \sigma_{i}\right)$ since $\psi_{k} \in f\left(\sigma, \sigma_{i}\right)$ but $f\left(\psi^{\prime}, \sigma_{i}\right)=$ $f\left(\psi, \sigma_{i}\right) \backslash \psi_{k}$. Thus, Lemma 3.2 implies that we must sort on $\sigma_{i}$ in order to achieve $\sigma$ order, a contradiction as we are given that $f\left(\sigma, \sigma_{i}\right) \subseteq f\left(\tau, \sigma_{i}\right)$.

Lemma 3.3 implies that if $f\left(\sigma, \sigma_{i}\right) \subseteq f\left(\tau, \sigma_{i}\right)$, a minimal sequence of sorts cannot involve non-bucketed sorts on modes in $f\left(\sigma, \sigma_{i}\right)$.

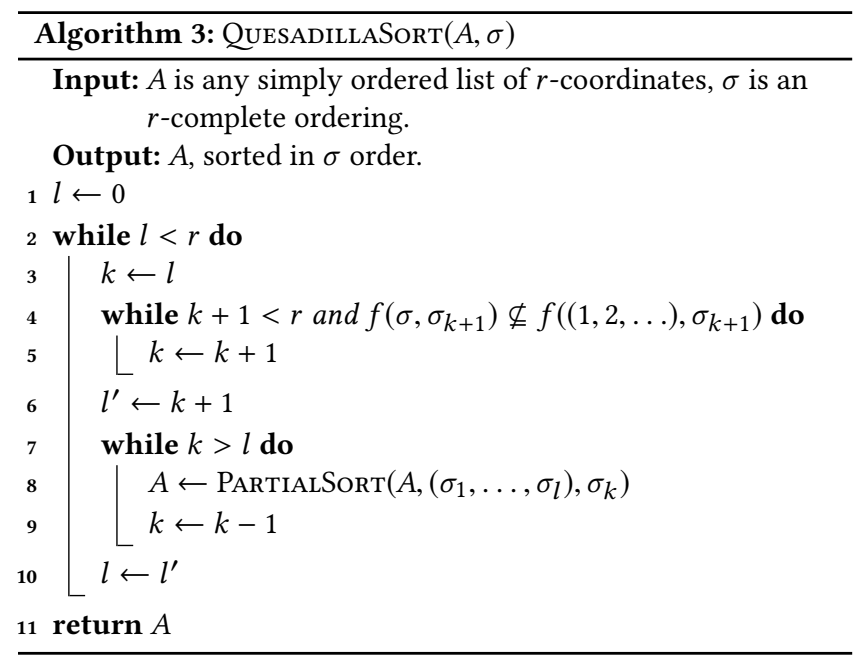

\subsection{Quesadilla Sort}

We now present the QUESADILLA algorithm for tensor transposition.

Theorem 3.1. Let A be a simply ordered list of $r$-coordinates. The sequence of sorts described by Quesadilla $(\sigma)$ will result in the $\sigma$ ordered list of coordinates in $A$.

Proof. We prove the result by showing that before and after each execution of the body of the loop on line 7, $A$ is sorted under a complete ordering $\tau$, where

$$
\left(\tau_{1}, \ldots, \tau_{l+l^{\prime}-(k+1)}\right)=\left(\sigma_{1}, \ldots, \sigma_{l}, \sigma_{k+1}, \ldots, \sigma_{l^{\prime}-1}\right),
$$

and the remaining modes of $\tau$ are in ascending order.

Before the first execution of our loop body, $A$ is simply ordered, $l=0$, and $l^{\prime}=k+1$. Thus, our claim is initially satisfied.

Assume our claim holds before some execution of the loop body. Let $A^{\prime}$ and $k^{\prime}$ be the values of $A$ and $k$ after executing the loop body. Let $t$ be the mode such that $\sigma_{k}=\tau_{t}$. Since $k>l$, (3) implies that $t>l+l^{\prime}-(k+1)$. Combining this observation with (1) and (2) leads to the observation that $A^{\prime}$ is sorted under the complete ordering $\tau^{\prime}$, where

$$
\tau^{\prime}=\left(\sigma_{1}, \ldots, \sigma_{l}, \sigma_{k}, \ldots, \sigma_{l^{\prime}-1}, \tau_{l+l^{\prime}-(k+1)}, \ldots, \tau_{t-1}, \tau_{t+1}, \ldots, \tau_{r}\right) .
$$

Therefore, (3) still holds for $\tau^{\prime}$ and $k^{\prime}$. Because $\left(\tau_{l+l^{\prime}-k}, \ldots, \tau_{r}\right)$ was ascending, $\left(\tau_{l+l^{\prime}-k^{\prime}}^{\prime}, \ldots, \tau_{r}^{\prime}\right)$ is also ascending. Thus, the claim holds after the execution of the loop body on line 7 .

All that remains to be shown is that our claim holds after we move through the loop on line 2. After leaving the line 7 loop, $k=l$ and $A$ is sorted under the complete ordering

$$
\tau=\left(\sigma_{1}, \ldots, \sigma_{l^{\prime}-1}, \tau_{l^{\prime}}, \ldots, \tau_{r}\right) .
$$

Notice that now $f\left(\sigma, \sigma_{k+1}\right) \subseteq f\left((1,2, \ldots), \sigma_{k+1}\right)$, either because that was the condition that stopped the loop on line 4 or because that loop stopped when $l^{\prime}=r$ and $f\left(\sigma, \sigma_{r}\right)=\emptyset$. Thus, for all $j>l^{\prime}$, $\sigma_{j}>\sigma_{l^{\prime}}$, and since $\left(\tau_{l^{\prime}}, \ldots, \tau_{r}\right)$ is ascending, $\tau_{l^{\prime}}=\sigma_{l^{\prime}}$. Thus, we set $l$ to $l^{\prime}$ and we have $\left(\tau_{1}, \ldots, \tau_{l}\right)=\left(\sigma_{1}, \ldots, \sigma_{l}\right)$ when we reach line 7 . The other claims will hold because $k+1$ will be equal to $l^{\prime}$. 
Theorem 3.2. Given a target ordering $\sigma$, QuesadillaSort $(\sigma)$ uses the minimum-length sequence of calls to PARTIALSORT required to sort any simply ordered list of $r$-coordinates to $\sigma$ order.

Proof. QuesadillaSort only calls PartialSort on modes $\sigma_{k}$ where $f\left(\sigma, \sigma_{k}\right) \nsubseteq f\left((1,2, \ldots), \sigma_{k}\right)$. Thus, Lemma 3.2 implies that QUESADILLASORT makes the minimum number of required calls to PartialSort.

TheOREM 3.3. Among minimum-length sequences of PARTIALSORT calls that sort simply ordered lists of $r$-coordinates to target ordering $\sigma$, the sequence used by QUESADILLASORT $(\sigma)$ minimizes the number of bucketed partial sorts.

Proof. The first execution of the loop on line 4 stops once $f\left(\sigma, \sigma_{k+1}\right) \subseteq f\left((1,2, \ldots), \sigma_{k+1}\right)$. For all $j>k+1$, we have $\sigma_{j} \in$ $f\left(\sigma, \sigma_{k+1}\right)$. Since QUESADILLA uses non-bucketed sorts for all sorts on modes $\sigma_{1}$ through $\sigma_{k+1}$, Lemma 3.3 implies that Quesadilla uses the minimum number of bucketed partial sorts possible when the total number of sorts is minimized.

\subsection{Top- $K$-sadilla Sort}

Although the two sorting primitives presented are both histogram sort variants, they could be replaced with any stable sort such as quicksort or merge sort. However, if a comparison sort is used at some level $k$ where the current ordering is $\tau$ and $\left(\tau_{1}, \ldots, \tau_{l}\right)=$ $\left(\sigma_{1}, \ldots, \sigma_{l}\right)$, it makes more sense to completely sort each bucket (equivalence class under $\left.\left(\tau_{1}, \ldots, \tau_{l}\right)\right)$ to $\sigma$ order.

Thus, we propose the Top- $K$-sAdilla algorithm, which uses QUesadilla to sort the tensor to $\left(\sigma_{1}, \ldots, \sigma_{K}\right)$ order, then sorts each bucket using quicksort. The best choice of the value $K$ will be investigated in our experiments, since it depends both on the permutation and on the dimension of the tensor.

\section{EVALUATION}

We evaluate QUESADILLA and Top- $K$-SADILlA sort against various state of the art approaches for sparse tensor transposition. As we will show, on the whole, our technique outperforms these existing approaches.

\subsection{Experimental Setup}

We created both parallel and serial implementations of our technique. We implemented the serial version in a code generator that emits $\mathrm{C}++$ code to transpose sparse tensors stored in the $\mathrm{COO}$ format using either QUESADILLA or Top- $K$-SADILLA sort. We implemented the parallel version by implementing parallel counting sort and bucketed counting sort primitives and calling the necessary sorts for QuEsADILLA. To implement the quicksort portion of Top- $K$-sAdilla, we identified the buckets in parallel and then sorted each bucket using the OpenMP for-loop parallelization construct. The buckets were scheduled using dynamic scheduling for Top-1-sadilla and guided scheduling for Top- $K$-sAdilla when $K$ $>1$. We made these scheduling choices because we expected more smaller buckets when buckets correspond to more coordinates. The overhead for dynamically scheduling many small buckets caused significant slow down.
Table 1: Statistics about tensors used in our experiments.

\begin{tabular}{lrl}
\hline \multicolumn{1}{c}{ Tensor } & Nonzeros & \multicolumn{1}{c}{ Dimensions } \\
\hline flickr-3d & 112890310 & $319686 \times 28153045 \times 1607191$ \\
nell-1 & 143599552 & $2902330 \times 2143368 \times 25495389$ \\
nell-2 & 76879419 & $12092 \times 9184 \times 28818$ \\
vast-2015-mc1-3d & 26021854 & $165427 \times 11374 \times 2$ \\
\hline chicago-crime-comm & 5330673 & $6186 \times 24 \times 77 \times 32$ \\
delicious-4d & 140126220 & $532924 \times 17262471 \times 2480308 \times 1443$ \\
enron & 54202099 & $6066 \times 5699 \times 44268 \times 1176$ \\
flickr-4d & 112890310 & $319686 \times 28153045 \times 1607191 \times 731$ \\
nips & 3101609 & $2482 \times 2862 \times 14036 \times 17$ \\
uber & 3309490 & $183 \times 24 \times 1140 \times 1717$ \\
\hline lbnl-network & 1698825 & $1605 \times 4198 \times 1631 \times 4209 \times 868131$ \\
vast-2015-mc1-5d & 26021945 & $165427 \times 11374 \times 2 \times 100 \times 89$ \\
\hline
\end{tabular}

Our serial implementation is available at https://github.com/ suzmue/taco/tree/transpose and our parallel implementation is available at https://github.com/suzmue/splatt.

To evaluate our technique, we compare it against SPLATT [23], a high-performance $\mathrm{C}++$ toolkit for sparse tensor factorization that uses a combination of histogram sort, quicksort, and insertion sort to sort tensors in COO. We also evaluate against sparse tensor transposition routines that sort nonzeros with (least significant digit) radix sort (using Algorithm 1 for each pass) or glibc's implementation of qsort.

We ran all experiments on a $2.5 \mathrm{GHz}$ Intel Xeon E5-2680 v3 machine with 24 cores, $30 \mathrm{MB}$ of L3 cache and $128 \mathrm{~GB}$ of main memory. The machine runs Ubuntu 18.04.3 LTS with glibc 2.27. We compiled the benchmarks using GCC 7.4.0. We ran each experiment 100 times and report minimum execution times.

We ran our experiments on real-world tensors obtained from the FROSTT Tensor Collection [22]. Table 1 reports statistics about these tensors. We stored tensors in the COO format and stored coordinates of nonzeros using 32-bit integers.

\subsection{Performance Evaluation}

For each tensor in Table 1, we measured the normalized running times of SPLATT, qsort, Top- $K$-SADILLA, Quesadilla, and radix sort for transposing the tensor from its initial ordering $\sigma=(1, \ldots, r)$ to every $r$ ! possible ordering. Figure 3 shows the results of these experiments aggregated over all 408 possible combinations of input tensors and output orderings. The appendix includes more detailed results that show the performance of each algorithm for every combination of input tensor and output ordering.

In serial tests, these results demonstrate that QuEsADILLA outperforms SPLATT, radix sort, and qsort on $60 \%$ of the sparse tensor transpositions. For half of all combinations, QUESADILLA is at least $1.19 \times$ faster than SPLATT, $1.68 \times$ faster than radix sort, and $2.76 \times$ faster than qsort. In parallel tests, at least one of QuEsADILLA or Top-2-SADILLA was the best strategy for $52 \%$ of all tensor and transposition combinations.

Quesadilla is able to significantly outperform radix sort by minimizing the number of passes over the input tensor. As Figure 4 shows, QUESADILLA exploits the partial ordering of the input tensor 


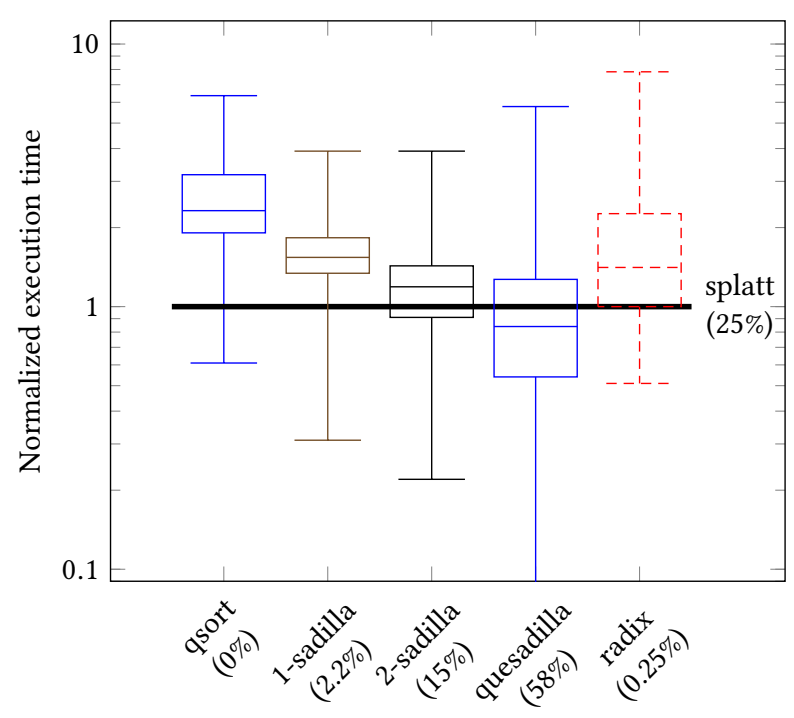

(a) serial

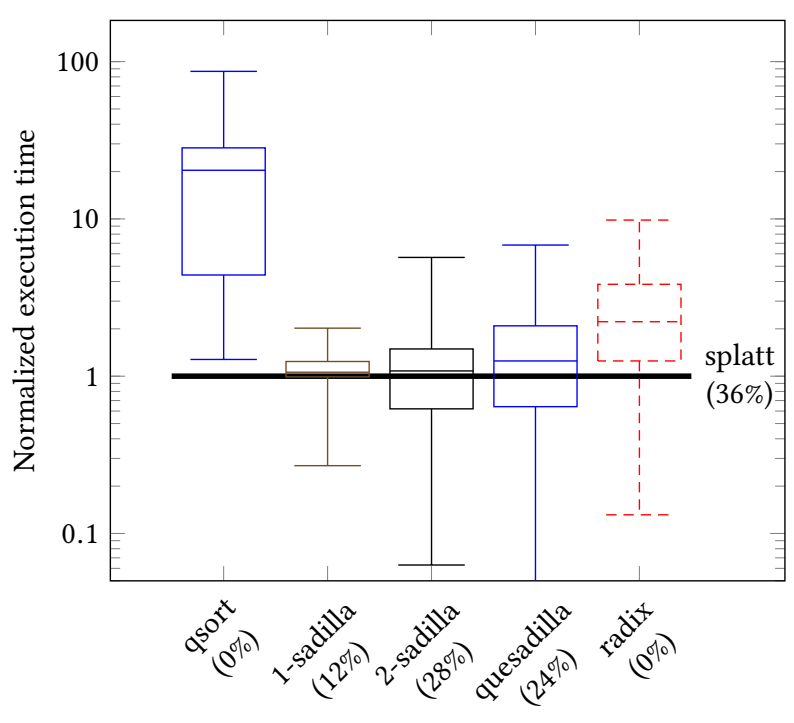

(b) parallel

Figure 3: Normalized execution times of sparse tensor transposition with various algorithms, aggregated over all 408 possible combinations of test tensors and output orderings. Percentages in parentheses indicate the proportion of combinations for which each algorithm is the fastest. Results are normalized to SPLATT (horizontal line) for each tensor and output ordering. ToP-1-SADILLA denotes Top- $K$-SADILLA with $K=1$. to eliminate at least one sorting pass for all possible output orderings and eliminate two or more passes for the majority of output orderings. By contrast, radix sort always makes as many sorting passes as there are modes in the input tensor, thereby incurring overhead from unnecessary memory traffic.

QUESADILLA's performance, however, depends to a large degree on the dimensions of the input tensor as well as the ordering of modes in the output. In particular, Quesadilla is more efficient when it does not have to sort large modes. Figure 5, for instance, shows QUESADILLA's performance for the lbnl-network tensor, whose last mode is significantly larger than the other modes. For output orderings where QuESADILLA does not have to sort the last mode, QUESADILLA significantly outperforms all other algorithms we evaluate. On the other hand, SPLATT and Top- $K$-SADILLA (where $K<r$ ) are more efficient for the other output orderings, with both being faster than QUESADILLA in approximately two-thirds of cases where QUESADILLA must sort the last mode in the serial implementation, and nearly all cases in the parallel implementation. This can be attributed to the fact that each invocation of PARTIALSORT in QUESADILLA requires a histogram containing $n_{k}$ bins, where $n_{k}$ is the size of the mode being sorted. When $n_{k}$ is large, accesses into the histogram are less likely to hit the cache, thereby limiting performance. Furthermore, constructing the histogram incurs $O\left(n_{k}\right)$ overhead, which becomes more significant when $n_{k}$ is large. Thus, as Figure 6 shows, PARTialSort is significantly slower for large modes than for small modes, assuming the bucketed dimensions are the same. This, in turn, limits QuESADILLA's performance for input tensors and output orderings that require sorting large modes. By contrast, SPLATT and Top- $K$-SADILLA use comparison-based sorting algorithms to sort all but the first mode or the first several modes respectively, thus making their performance less dependent on the dimensions of the input tensor.

When $K=1$, Top- $K$-sAdilla reduces to the Top-1-sAdilla algorithm that is similar to what SPLATT implements for sorting COO tensors, which we summarize in Section 2.2. Unlike SPLATT, which uses a custom hand-optimized implementation of quicksort, serial Top-1-SADILLA uses qsort from $\mathrm{C}$ stdlib to sort nonzeros within each bucket created by the initial histogram sort. As Figure 3 shows, serial SPLATT outperforms serial Top-1-SADILLA for most tensor transpositions in our experiments, thereby demonstrating that SPLATT's custom implementation of quicksort is more efficient than qsort. This performance difference suggests we can improve Top- $K$-SADILLA's performance by using more optimized implementations of comparison sort to sort each bucket.

\section{CONCLUSION}

We have described an algorithm to transpose sparse tensors faster than simply sorting a list of coordinates. By taking advantage of the lexicographic ordering of the input and knowledge of the requested transposition, our algorithm applies only a subset of the passes of a radix sort and thereby reduces the amount of work required to sort the coordinates. We provide two non-comparison based partial sorting algorithms for radix sort passes that are optimized for different situations. We prove two things: (1) We prove that our algorithm minimizes the total calls to either sorting algorithm. (2) We prove that among sorts with the minimum total calls, we 


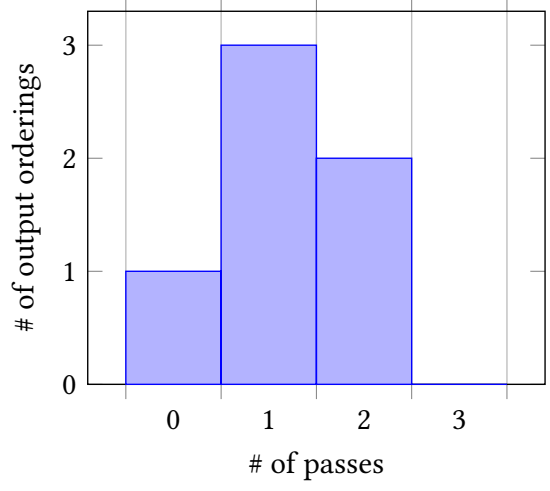

(a) 3-tensors

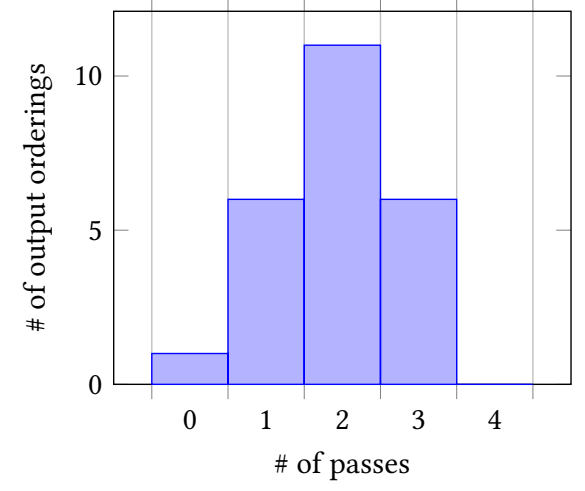

(b) 4-tensors

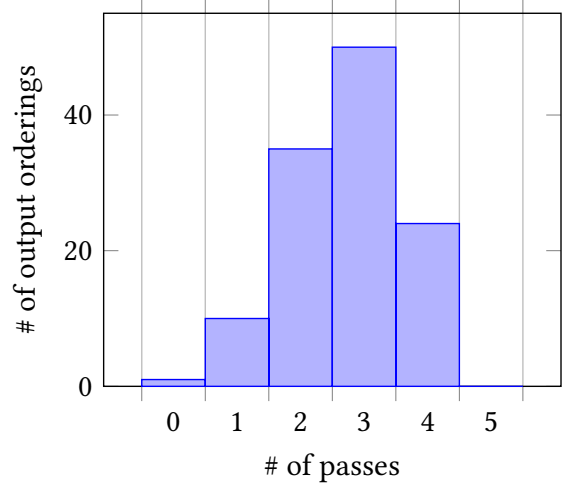

(c) 5-tensors

Figure 4: Distributions of the number of sorting passes needed by QUESADILLA to transpose tensors of varying rank.

minimize the number of calls to the more expensive of the two. The amount of work required by our algorithm is proportional to the number of modes that need reordering in the transposition. We evaluated our algorithm empirically with a $\mathrm{C}++$ implementation, and showed that it produced significant improvements over existing approaches.

As sparse tensor representations receive increasing study, diversity in tensor formats will increase and applications will more frequently convert between formats. Sparse tensor transposition is the most basic instance of sparse format conversion, and an important subroutine in several format conversions. We have provided evidence that naive algorithms for sparse tensor transpositions can be improved substantially, but there are further improvements that need investigation.

Focusing on the multi-pass coordinate-sorting-based transposition technique we describe, improvements can be made in scheduling passes, the implementation of passes themselves, and handling the buckets. Although we minimize the number of passes over the data, we don't necessarily pick a schedule of passes that minimizes the true runtime. Since the bucketed histogram sort costs more than the histogram sort, we can improve our scheduling by minimizing a cost model which reflects the true costs of the passes.

We can improve the implementation of a sorting pass by reducing the size of coordinates using bit-packing techniques. If the mode to be sorted has a large dimension, it can make sense to perform the histogram sort itself as a radix sort, with multiple passes and a radix smaller than the dimension. In some cases, we can also fuse the first loop of the next histogram sort into the last loop of the current one, reducing the number of reads.

Discovering the buckets is expensive. If we need to perform several bucketed histogram sorts with the same buckets, we only need to discover the buckets once at the beginning, perform the histogram sorts, and then sort on the buckets at the end, skipping the bucketing step between the two sorts. Since $l$ is constant, we can use the same buckets for all iterations of the loop on line 7 of Algorithm 3. Additionally, instead of evaluating the all $l$ entries of each coordinate to discover the buckets, we can use buckets from the previous pass, which differ precisely when the previous entries differed. While such an optimization would involve permuting a bucket array, we can avoid examining entire coordinates during bucket discovery, saving a factor of $r$ in the asymptotic analysis.

If our goal is to transpose tensors stored in formats other than COO, including formats like HiCOO [18], BICRS [32], and JAD [21], additional optimizations may present themselves. For example, instead of converting to coordinates, then sorting, our first histogram sort can iterate over the input format in order, fusing the conversion to coordinates into the first histogram sort. Additionally, the sorting techniques we describe in this work may apply directly to the format we want to transpose. If the tensor is in CSF, for example, it may be possible to sort the nodes in the CSF tree directly, moving the nodes instead of moving entire subtrees.

\section{ACKNOWLEDGMENTS}

This work was supported by a grant from the Toyota Research Institute, DARPA PAPPA Grant HR00112090017, and a DOE CSGF Fellowship DE-FG02-97ER25308.

\section{REFERENCES}

[1] Martín Abadi, Ashish Agarwal, Paul Barham, Eugene Brevdo, Zhifeng Chen, Craig Citro, Greg S. Corrado, Andy Davis, Jeffrey Dean, Matthieu Devin, Sanjay Ghemawat, Ian Goodfellow, Andrew Harp, Geoffrey Irving, Michael Isard, Yangqing Jia, Rafal Jozefowicz, Lukasz Kaiser, Manjunath Kudlur, Josh Levenberg, Dan Mane, Rajat Monga, Sherry Moore, Derek Murray, Chris Olah, Mike Schuster, Jonathon Shlens, Benoit Steiner, Ilya Sutskever, Kunal Talwar, Paul Tucker, Vincent Vanhoucke, Vijay Vasudevan, Fernanda Viegas, Oriol Vinyals, Pete Warden, Martin Wattenberg, Martin Wicke, Yuan Yu, and Xiaoqiang Zheng. 2016. TensorFlow: Large-Scale Machine Learning on Heterogeneous Distributed Systems. arXiv:1603.04467 [cs] (March 2016). http://arxiv.org/abs/1603.04467 arXiv: 1603.04467.

[2] Brett W. Bader and Tamara G. Kolda. 2007. Efficient MATLAB Computations with Sparse and Factored Tensors. SIAM Journal on Scientific Computing 30, 1 (Jan. 2007), 205-231. https://doi.org/10.1137/060676489

[3] Guy E. Blelloch, Charles E. Leiserson, Bruce M. Maggs, C. Greg Plaxton, Stephen J. Smith, and Marco Zagha. 1991. A comparison of sorting algorithms for the connection machine CM-2. In Proceedings of the third annual ACM symposium on Parallel algorithms and architectures (SPAA '91). Association for Computing Machinery, Hilton Head, South Carolina, USA, 3-16. https://doi.org/10.1145/ 113379.113380

[4] Aydin Buluc and John R. Gilbert. 2008. On the representation and multiplication of hypersparse matrices. In 2008 IEEE International Symposium on Parallel and Distributed Processing. 1-11. https://doi.org/10.1109/IPDPS.2008.4536313 ISSN: 1530-2075 


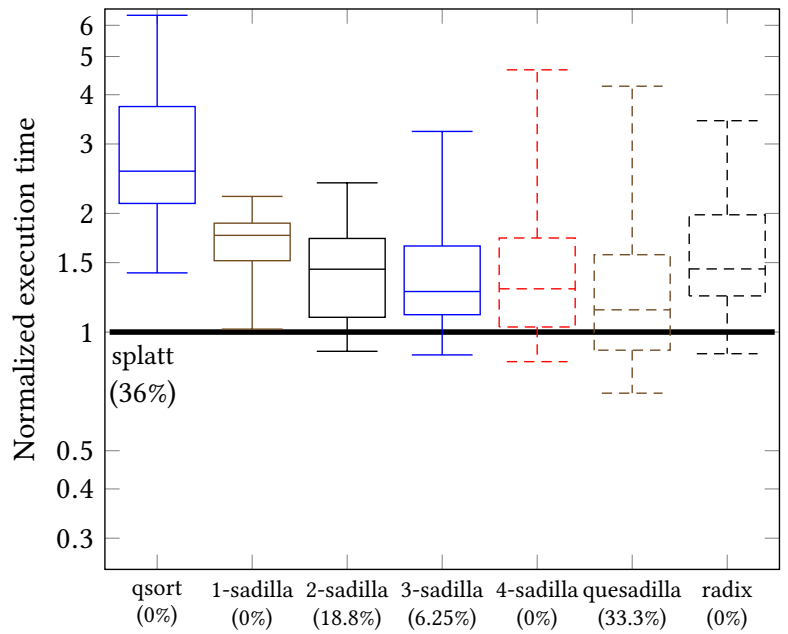

(a) Sort required on last mode (serial)

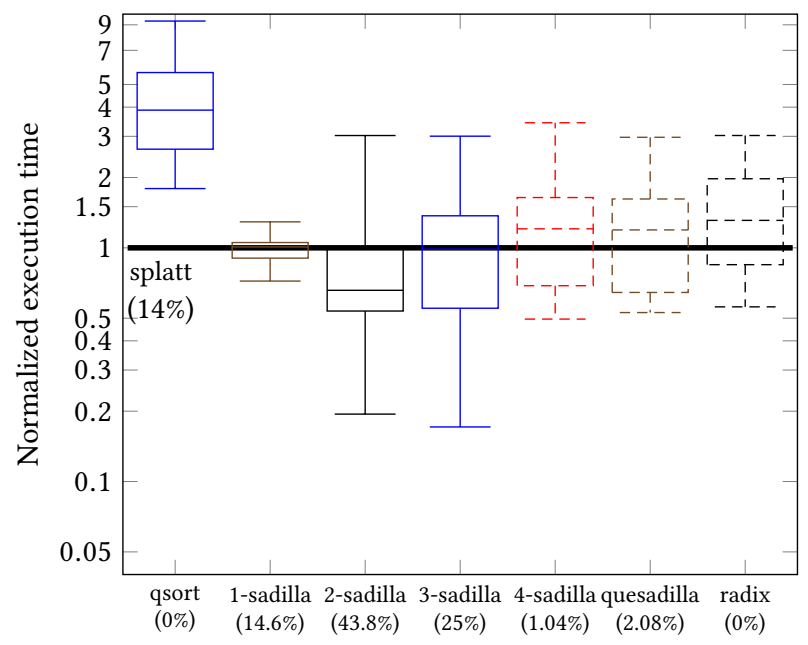

(c) Sort required on last mode (parallel)

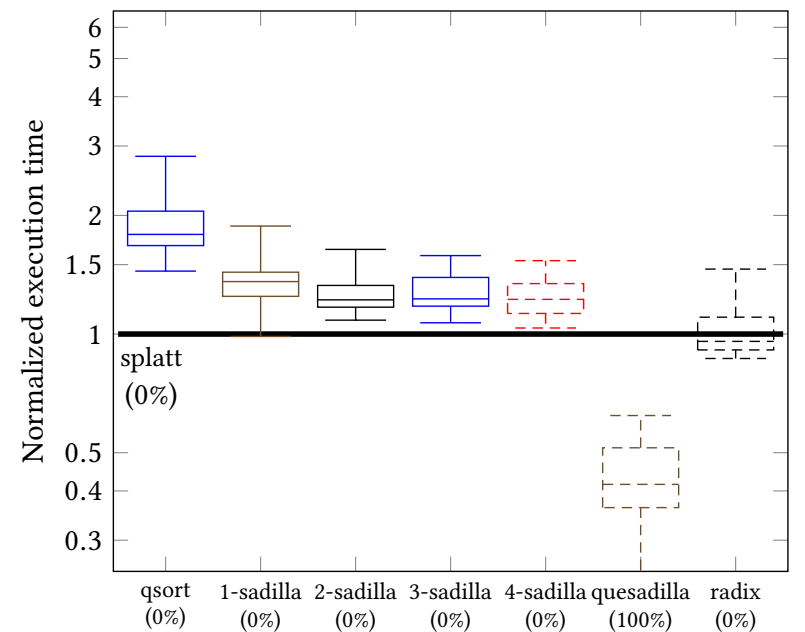

(b) No sort required on last mode (serial)

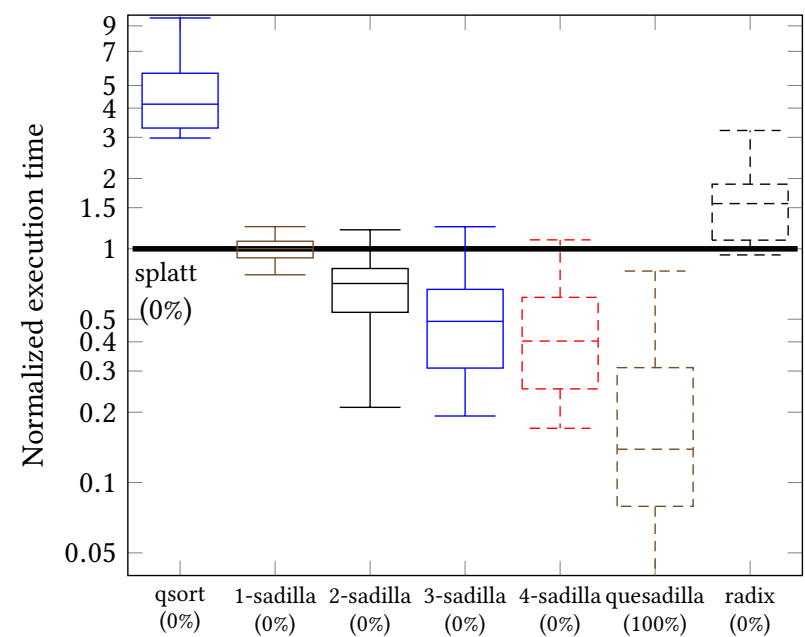

(d) No sort required on last mode (parallel)

Figure 5: Normalized execution times of sparse tensor transposition with various algorithms for the lbnl-network tensor, aggregated over (a) all output orderings where QUESADILLA needs to sort on the last mode and (b) all output orderings where the last mode does not need to be sorted. Percentages in parentheses indicate the proportion of combinations for which each algorithm is the fastest. Results are normalized to SPLATT (horizontal lines) for each tensor and output ordering. Again, e.g. Top-1-SADILla denotes Top- $K$-SADILLA with $K=1$.

[5] Frank Cameron. 1993. Two space-saving algorithms for computing the permuted transpose of a sparse matrix. Advances in Engineering Software 17, 1 (Jan. 1993) 49-60. https://doi.org/10.1016/0965-9978(93)90041-Q

[6] Bryan Catanzaro, Alexander Keller, and Michael Garland. 2014. A decomposition for in-place matrix transposition. ACM SIGPLAN Notices 49, 8 (Feb. 2014), 193-206. https://doi.org/10.1145/2692916.2555253

[7] Thomas H. Cormen, Charles E. Leiserson, Ronald L. Rivest, and Clifford Stein. 2009. Introduction to algorithms (3rd ed ed.). MIT Press, Cambridge, Mass. OCLC: ocn311310321.

[8] S. C. Eisenstat, M. C. Gursky, M. H. Schultz, and A. H. Sherman. 1982. Yale sparse matrix package I: The symmetric codes. Internat. 7. Numer. Methods Engrg. 18, 8 (1982), 1145-1151. https://doi.org/10.1002/nme.1620180804

[9] Miguel A. Gonzalez-Mesa, Eladio D. Gutierrez, and Oscar Plata. 2013. Parallelizing the Sparse Matrix Transposition: Reducing the Programmer Effort Using Transactional Memory. Procedia Computer Science 18 (Jan. 2013), 501-510. https://doi.org/10.1016/j.procs.2013.05.214
[10] Song Guo, Yong Dou, Yuanwu Lei, Qiang Wang, Fei Xia, and Jianning Chen. 2016. Designing Parallel Sparse Matrix Transposition Algorithm Using ELLPACK-R for GPUs. In Computer Engineering and Technology (Communications in Computer and Information Science), Weixia Xu, Liquan Xiao, Jinwen Li, and Chengyi Zhang (Eds.). Springer, Berlin, Heidelberg, 61-68. https://doi.org/10.1007/978-3-66249283-3_7

[11] Fred Gustavson, Lars Karlsson, and Bo Kågström. 2012. Parallel and CacheEfficient In-Place Matrix Storage Format Conversion. ACM Transactions on Mathematical Software (TOMS) 38, 3 (April 2012), 17:1-17:32. https://doi.org/10. $1145 / 2168773.2168775$

[12] Fred G. Gustavson. 1978. Two Fast Algorithms for Sparse Matrices: Multiplication and Permuted Transposition. ACM Transactions on Mathematical Software (TOMS) 4, 3 (Sept. 1978), 250-269. https://doi.org/10.1145/355791.355796

[13] Eun-Jin Im and Katherine Yelick. 2001. Optimizing Sparse Matrix Computations for Register Reuse in SPARSITY. In Computational Science - ICCS 2001, Vassil N. Alexandrov, Jack J. Dongarra, Benjoe A. Juliano, René S. Renner, and C. J. Kenneth 


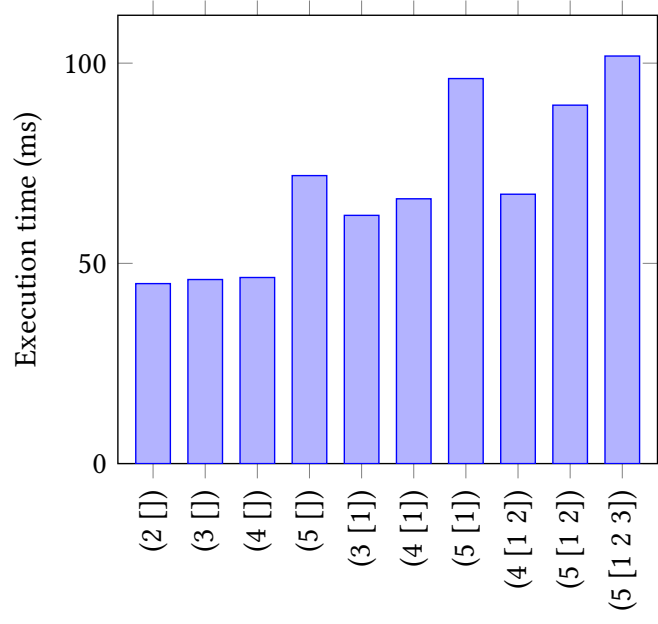

(a) lbnl-network (serial)

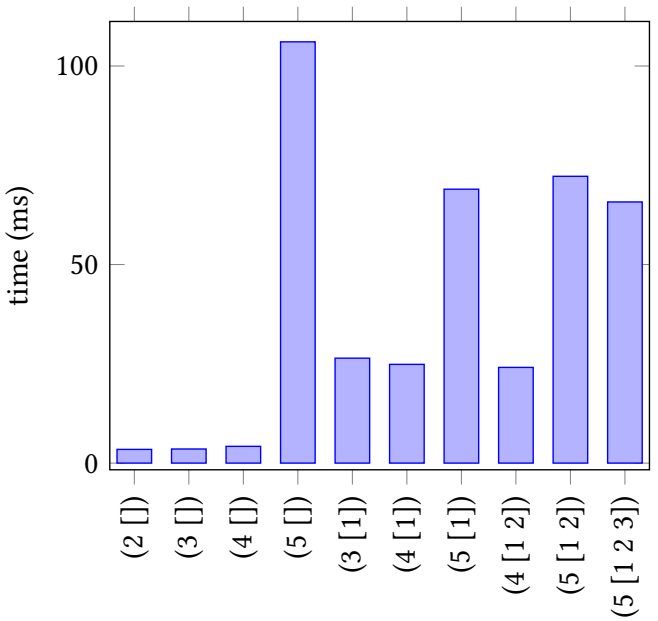

sort

(c) lbnl-network (parallel)

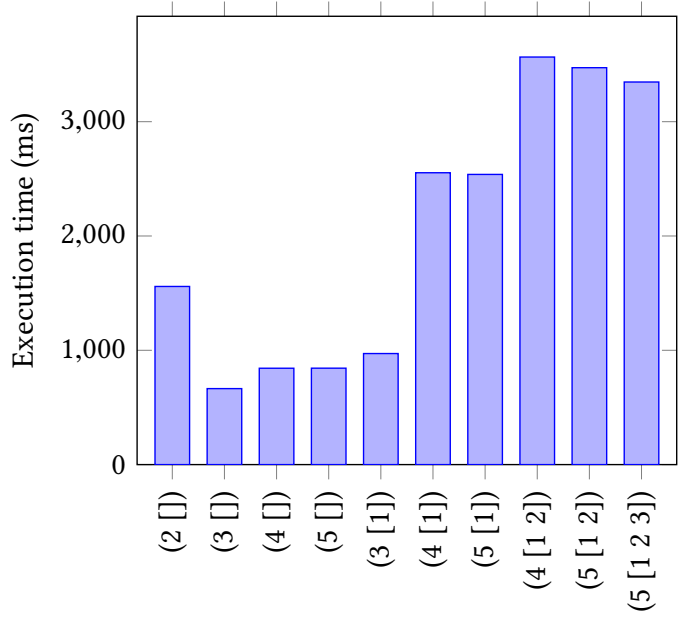

(b) vast-2015-mc1-5d (serial)

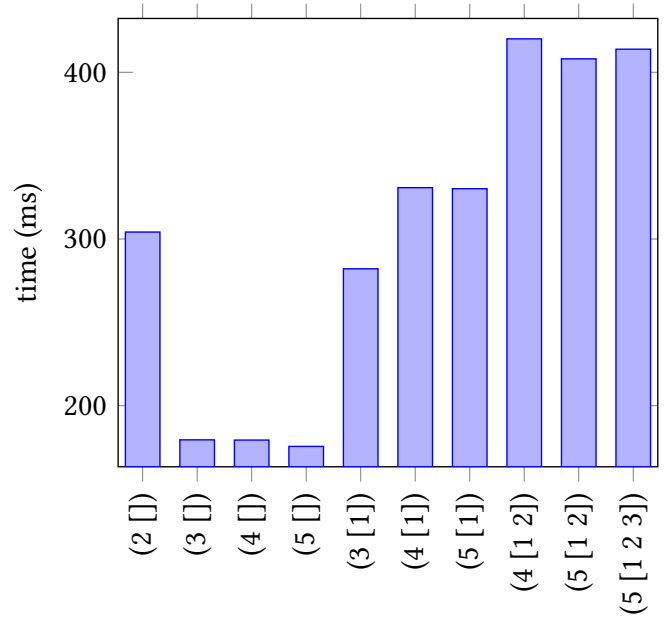

sort

(d) vast-2015-mc1-5d (parallel)

Figure 6: Execution times of PARTIALSORT for sorting different modes of two test tensors. Labels along the $x$-axes indicate the modes being sorted; for instance, (4 [ 12 2]) denotes sort on mode 4 assuming modes 1 and 2 are bucketed.

Tan (Eds.). Springer Berlin Heidelberg, Berlin, Heidelberg, 127-136.

[14] Lars Karlsson. 2009. Blocked in-place transposition with application to storage format conversion. Technical Report.

[15] S. D. Kaushik, C. . Huang, J. R. Johnson, R. W. Johnson, and P. Sadayappan. 1993. Efficient transposition algorithms for large matrices. In Supercomputing '93:Proceedings of the 1993 ACM/IEEE Conference on Supercomputing. 656-665. https://doi.org/10.1109/SUPERC.1993.1263520

[16] Fredrik Kjolstad, Peter Ahrens, Shoaib Kamil, and Saman Amarasinghe. 2018 Sparse Tensor Algebra Optimizations with Workspaces. arXiv:1802.10574 [cs] (April 2018). http://arxiv.org/abs/1802.10574 arXiv: 1802.10574

[17] Jiajia Li, Yuchen Ma, Xiaolong Wu, Ang Li, and Kevin Barker. 2019. PASTA a parallel sparse tensor algorithm benchmark suite. CCF Transactions on High Performance Computing 1, 2 (Aug. 2019), 111-130. https://doi.org/10.1007/s42514019-00012-w

[18] Jiajia Li, Jimeng Sun, and Richard Vuduc. 2018. HiCOO: Hierarchical Storage of Sparse Tensors. In SC18: International Conference for High Performance Computing, Networking, Storage and Analysis. 238-252. https://doi.org/10.1109/SC.2018.00022 ISSN: null.
[19] Omar Obeya, Endrias Kahssay, Edward Fan, and Julian Shun. 2019. TheoreticallyEfficient and Practical Parallel In-Place Radix Sorting. In The 31st ACM Symposium on Parallelism in Algorithms and Architectures (SPAA '19). Association for Computing Machinery, Phoenix, AZ, USA, 213-224. https://doi.org/10.1145/3323165. 3323198

[20] Greg Ruetsch and Paulius Micikevicius. 2009. Optimizing matrix transpose in CUDA. Technical Report.

[21] Youcef. Saad. 1989. Krylov Subspace Methods on Supercomputers. SIAM 7. Sci. Statist. Comput. 10, 6 (Nov. 1989), 1200-1232. https://doi.org/10.1137/0910073

[22] Shaden Smith, Jee W. Choi, Jiajia Li, Richard Vuduc, Jongsoo Park, Xing Liu, and George Karypis. 2017. FROSTT: The Formidable Repository of Open Sparse Tensors and Tools. http://frostt.io/

[23] Shaden Smith, Niranjay Ravindran, Nicholas D. Sidiropoulos, and George Karypis. 2015. SPLATT: Efficient and Parallel Sparse Tensor-Matrix Multiplication. In Proceedings of the 2015 IEEE International Parallel and Distributed Processing Symposium (IPDPS '15). IEEE Computer Society, Washington, DC, USA, 61-70. https://doi.org/10.1109/IPDPS.2015.27 
[24] Paul Springer, Jeff R. Hammond, and Paolo Bientinesi. 2017. TTC: A HighPerformance Compiler for Tensor Transpositions. ACM Transactions on Mathematical Software (TOMS) 44, 2 (Aug. 2017), 15:1-15:21. https://doi.org/10.1145/ 3104988

[25] I. Sung, G. D. Liu, and W. W. Hwu. 2012. DL: A data layout transformation system for heterogeneous computing. In 2012 Innovative Parallel Computing (InPar). 1-11. https://doi.org/10.1109/InPar.2012.6339606

[26] I-Jui Sung, Juan Gómez-Luna, José María González-Linares, Nicolás Guil, and Wen-Mei W. Hwu. 2014. In-place transposition of rectangular matrices on accelerators. ACM SIGPLAN Notices 49, 8 (Feb. 2014), 207-218. https://doi.org/10 $1145 / 2692916.2555266$

[27] Andrey Vladimirov. 2013. Multithreaded Transposition of Square Matrices with Common Code for Intel Xeon Processors and Intel Xeon Phi Coprocessors. Technical Report.

[28] F. Vázquez, J. J. Fernández, and E. M. Garzón. 2011. A new approach for sparse matrix vector product on NVIDIA GPUs. Concurrency and Computation: Practice and Experience 23, 8 (2011), 815-826. https://doi.org/10.1002/cpe.1658 arXiv:https://onlinelibrary.wiley.com/doi/pdf/10.1002/cpe.1658

[29] Hao Wang, Weifeng Liu, Kaixi Hou, and Wu-chun Feng. 2016. Parallel Transposition of Sparse Data Structures. In Proceedings of the 2016 International Conference on Supercomputing (ICS '16). Association for Computing Machinery, Istanbul, Turkey, 1-13. https://doi.org/10.1145/2925426.2926291

[30] Tien-Hsiung Weng, Delgerdalai Batjargal, Hoa Pham, Meng-Yen Hsieh, and KuanChing Li. 2013. Parallel Matrix Transposition and Vector Multiplication Using OpenMP. In Intelligent Technologies and Engineering Systems (Lecture Notes in Electrical Engineering), Jengnan Juang and Yi-Cheng Huang (Eds.). Springer, New York, NY, 243-249. https://doi.org/10.1007/978-1-4614-6747-2_30

[31] Tien-Hsiung Weng, Hoa Pham, Hai Jiang, and Kuan-Ching Li. 2013. Designing Parallel Sparse Matrix Transposition Algorithm Using CSR for GPUs. In Intelligent Technologies and Engineering Systems (Lecture Notes in Electrical Engineering), Jengnan Juang and Yi-Cheng Huang (Eds.). Springer, New York, NY, 251-257. https://doi.org/10.1007/978-1-4614-6747-2_31

[32] Albert-Jan N. Yzelman and Rob H. Bisseling. 2012. A Cache-Oblivious Sparse Matrix-Vector Multiplication Scheme Based on the Hilbert Curve. In Progress in Industrial Mathematics at ECMI 2010 (Mathematics in Industry), Michael Günther, Andreas Bartel, Markus Brunk, Sebastian Schöps, and Michael Striebel (Eds.) Springer, Berlin, Heidelberg, 627-633. https://doi.org/10.1007/978-3-642-25100973

[33] Keliang Zhang and Baifeng Wu. 2012. A Novel Parallel Approach of Radix Sort with Bucket Partition Preprocess. In 2012 IEEE 14th International Conference on High Performance Computing and Communication 2012 IEEE 9th International Conference on Embedded Software and Systems. 989-994. https://doi.org/10.1109/ HPCC.2012.144 


\section{A AGGREGATE RESULTS}

These tables contain statistics about the performance of the algorithms across all permutations and tensors. In addition, we counted the number of times that each strategy was the best of all of the strategies. We exclude Top-2-sadilla and Top-3-sadilla from these results, as the strategy is not comparable across tensors of different orders. 
Table 2: Aggregate timing results (serial)

\begin{tabular}{|c|c|c|c|c|c|c|}
\hline stat & qsort & splatt & 1-sadilla & 2-sadilla & quesadilla & radix \\
\hline min & 0.61 & 1.00 & 0.31 & 0.22 & 0.00 & 0.51 \\
Q1 & 1.91 & 1.00 & 1.34 & 0.91 & 0.54 & 1.00 \\
median & 2.32 & 1.00 & 1.54 & 1.19 & 0.84 & 1.41 \\
Q3 & 3.18 & 1.00 & 1.83 & 1.43 & 1.27 & 2.26 \\
max & 6.36 & 1.00 & 3.91 & 3.91 & 5.78 & 7.84 \\
wins & 0 & 25 & 2.2 & 15 & 58 & 0.25 \\
\hline
\end{tabular}

Table 3: Aggregate timing results (parallel)

\begin{tabular}{|c|c|c|c|c|c|c|}
\hline stat & qsort & splatt & 1-sadilla & 2-sadilla & quesadilla & radix \\
\hline min & 1.27 & 1 & 0.27 & 0.063 & 0.00 & 0.13 \\
Q1 & 4.40 & 1.00 & 0.99 & 0.62 & 0.64 & 1.25 \\
median & 20.38 & 1.00 & 1.06 & 1.08 & 1.25 & 2.22 \\
Q3 & 28.29 & 1.00 & 1.24 & 1.49 & 2.09 & 3.84 \\
max & 86.70 & 1.00 & 2.02 & 5.69 & 6.82 & 9.83 \\
wins & 0 & 36 & 12 & 28 & 24 & 0 \\
\hline
\end{tabular}

Table 4: Median results by tensor (serial)

\begin{tabular}{|c|c|c|c|c|c|c|}
\hline filename & qsort & splatt & 1-sadilla & 2-sadilla & quesadilla & radix \\
\hline flickr-3d & 4.31 & 1.00 & 1.71 & 1.45 & 0.99 & 2.40 \\
nell-1 & 3.04 & 1.00 & 1.94 & 1.15 & 0.81 & 3.08 \\
nell-2 & 2.80 & 1.00 & 1.90 & 1.35 & 0.60 & 1.34 \\
vast-2015-mc1-3d & 3.49 & 1.00 & 1.49 & 1.18 & 0.55 & 2.07 \\
chicago-crime-comm & 2.18 & 1.00 & 1.71 & 1.08 & 0.52 & 0.93 \\
delicious-4d & 2.66 & 1.00 & 1.51 & 1.11 & 1.17 & 1.89 \\
enron & 2.43 & 1.00 & 1.53 & 1.06 & 0.64 & 1.18 \\
flickr-4d & 3.48 & 1.00 & 1.54 & 1.21 & 1.11 & 2.02 \\
nips & 2.84 & 1.00 & 1.88 & 1.35 & 0.81 & 1.79 \\
uber & 2.24 & 1.00 & 1.68 & 1.30 & 0.63 & 1.10 \\
lbnl-network & 2.29 & 1.00 & 1.67 & 1.34 & 1.04 & 1.37 \\
vast-2015-mc1-5d & 1.97 & 1.00 & 1.35 & 0.92 & 0.75 & 1.25 \\
\hline
\end{tabular}

Table 5: Median by tensor (parallel)

\begin{tabular}{|c|c|c|c|c|c|c|}
\hline filename & qsort & splatt & 1-sadilla & 2-sadilla & quesadilla & radix \\
\hline flickr-3d & 32.39 & 1.00 & 1.06 & 1.03 & 0.90 & 5.52 \\
nell-1 & 22.94 & 1.00 & 1.08 & 1.05 & 0.88 & 6.33 \\
nell-2 & 32.69 & 1.00 & 1.29 & 1.52 & 1.35 & 2.69 \\
vast-2015-mc1-3d & 25.64 & 1.00 & 1.30 & 0.93 & 0.60 & 2.45 \\
chicago-crime-comm & 19.41 & 1.00 & 1.25 & 0.93 & 0.84 & 1.82 \\
delicious-4d & 27.22 & 1.00 & 1.15 & 1.52 & 1.88 & 4.85 \\
enron & 28.81 & 1.00 & 1.28 & 1.68 & 1.61 & 3.20 \\
flickr-4d & 27.69 & 1.00 & 1.11 & 1.49 & 1.74 & 5.73 \\
nips & 40.06 & 1.00 & 1.43 & 1.37 & 1.24 & 2.02 \\
uber & 41.10 & 1.00 & 1.60 & 1.53 & 0.95 & 1.70 \\
lbnl-network & 3.97 & 1.00 & 1.01 & 0.68 & 1.01 & 1.33 \\
vast-2015-mc1-5d & 23.18 & 1.00 & 1.05 & 1.28 & 1.84 & 3.06 \\
\hline
\end{tabular}


Table 6: Wins by tensor (serial)

\begin{tabular}{|c|c|c|c|c|c|c|}
\hline filename & qsort & splatt & 1-sadilla & 2-sadilla & quesadilla & radix \\
\hline flickr-3d & $0 \%$ & $50 \%$ & $0 \%$ & $0 \%$ & $50 \%$ & $0 \%$ \\
nell-1 & $0 \%$ & $33.3 \%$ & $0 \%$ & $0 \%$ & $66.7 \%$ & $0 \%$ \\
nell-2 & $0 \%$ & $33.3 \%$ & $0 \%$ & $0 \%$ & $66.7 \%$ & $0 \%$ \\
vast-2015-mc1-3d & $0 \%$ & $0 \%$ & $0 \%$ & $0 \%$ & $100 \%$ & $0 \%$ \\
chicago-crime-comm & $0 \%$ & $12.5 \%$ & $0 \%$ & $0 \%$ & $87.5 \%$ & $0 \%$ \\
delicious-4d & $0 \%$ & $45.8 \%$ & $0 \%$ & $16.7 \%$ & $37.5 \%$ & $0 \%$ \\
enron & $0 \%$ & $12.5 \%$ & $0 \%$ & $4.17 \%$ & $79.2 \%$ & $4.17 \%$ \\
flickr-4d & $0 \%$ & $45.8 \%$ & $0 \%$ & $12.5 \%$ & $41.7 \%$ & $0 \%$ \\
nips & $0 \%$ & $25 \%$ & $12.5 \%$ & $8.33 \%$ & $54.2 \%$ & $0 \%$ \\
uber & $0 \%$ & $16.7 \%$ & $0 \%$ & $0 \%$ & $83.3 \%$ & $0 \%$ \\
lbnl-network & $0 \%$ & $36.7 \%$ & $0 \%$ & $16.7 \%$ & $46.7 \%$ & $0 \%$ \\
vast-2015-mc1-5d & $0 \%$ & $10.8 \%$ & $5 \%$ & $25.8 \%$ & $58.3 \%$ & $0 \%$ \\
\hline
\end{tabular}

Table 7: Wins by tensor (parallel)

\begin{tabular}{|c|c|c|c|c|c|c|}
\hline filename & qsort & splatt & 1-sadilla & 2-sadilla & quesadilla & radix \\
\hline flickr-3d & $0 \%$ & $16.7 \%$ & $16.7 \%$ & $0 \%$ & $66.7 \%$ & $0 \%$ \\
nell-1 & $0 \%$ & $16.7 \%$ & $16.7 \%$ & $16.7 \%$ & $50 \%$ & $0 \%$ \\
nell-2 & $0 \%$ & $50 \%$ & $16.7 \%$ & $0 \%$ & $33.3 \%$ & $0 \%$ \\
vast-2015-mc1-3d & $0 \%$ & $33.3 \%$ & $16.7 \%$ & $0 \%$ & $50 \%$ & $0 \%$ \\
chicago-crime-comm & $0 \%$ & $8.33 \%$ & $16.7 \%$ & $20.8 \%$ & $54.2 \%$ & $0 \%$ \\
delicious-4d & $0 \%$ & $50 \%$ & $16.7 \%$ & $12.5 \%$ & $20.8 \%$ & $0 \%$ \\
enron & $0 \%$ & $79.2 \%$ & $0 \%$ & $0 \%$ & $20.8 \%$ & $0 \%$ \\
flickr-4d & $0 \%$ & $41.7 \%$ & $16.7 \%$ & $20.8 \%$ & $20.8 \%$ & $0 \%$ \\
nips & $0 \%$ & $62.5 \%$ & $4.17 \%$ & $8.33 \%$ & $25 \%$ & $0 \%$ \\
uber & $0 \%$ & $37.5 \%$ & $0 \%$ & $8.33 \%$ & $54.2 \%$ & $0 \%$ \\
lbnl-network & $0 \%$ & $11.7 \%$ & $11.7 \%$ & $53.3 \%$ & $23.3 \%$ & $0 \%$ \\
vast-2015-mc1-5d & $0 \%$ & $49.2 \%$ & $15 \%$ & $26.7 \%$ & $9.17 \%$ & $0 \%$ \\
\hline
\end{tabular}




\section{B DETAILED RESULTS}

These tables contain the results of running all of the experiments. They are organized by file and the permutations are ordered lexicographically. A cell that contains a value of 1 is colored white. This value means that it performed as well as SPLATT. A cell that contains a value $>1$ is colored red and performed worse than SPLATT. A cell that contains a value $<1$ is colored blue and performed better than SPLATT. 
Figure 7: flickr-3d results normalized by splatt (serial)

\begin{tabular}{|c|c|c|c|c|c|c|}
\hline & qsort & splatt & 1-sadilla & 2-sadilla & quesadilla & radix \\
\hline 123 & 4.12 & 1 & 1.84 & 0.56 & 0 & 5.59 \\
\hline 132 & 2.75 & 1 & 1.56 & 1.47 & 1.01 & 1.85 \\
\hline 213 & 4.4 & 1 & 1.4 & 1.43 & 0.97 & 2.44 \\
\hline 231 & 4.87 & 1 & 1.58 & 3.37 & 3.09 & 3.41 \\
\hline 312 & 5.48 & 1 & 2.07 & 1.38 & 0.75 & 2.35 \\
\hline 321 & 4.22 & 1 & 2 & 1.56 & 1.4 & 1.66 \\
\hline
\end{tabular}

Figure 8: nell-1 results normalized by splatt (serial)

\begin{tabular}{|c|c|c|c|c|c|c|}
\hline & qsort & splatt & 1-sadilla & 2-sadilla & quesadilla & radix \\
\hline 123 & 4.22 & 1 & 1.99 & 0.96 & 0 & 7.84 \\
\hline 132 & 3.12 & 1 & 1.89 & 3.91 & 3.69 & 3.8 \\
\hline 213 & 4.23 & 1 & 2.14 & 1.34 & 0.7 & 4.83 \\
\hline 231 & 2.96 & 1 & 2.05 & 2.51 & 2.36 & 2.37 \\
\hline 312 & 1.77 & 1 & 1.01 & 0.88 & 0.77 & 1.16 \\
\hline 321 & 1.7 & 1 & 1.08 & 0.93 & 0.84 & 0.91 \\
\hline
\end{tabular}

Figure 9: nell-2 results normalized by splatt (serial)

\begin{tabular}{l|c|c|c|ccc} 
& qsort & splatt & 1-sadilla & 2-sadilla & quesadilla & radix \\
123 & 4.15 & 1 & 2.41 & 1.34 & 0 & 4.15 \\
132 & 2.5 & 1 & 1.8 & 1.62 & 1.34 & 1.47 \\
213 & 5.5 & 1 & 2.87 & 1.76 & 0.52 & 3.6 \\
231 & 2.55 & 1 & 1.99 & 1.37 & 1.09 & 1.22 \\
312 & 3.04 & 1 & 1.36 & 0.92 & 0.65 & 0.95 \\
321 & 2.26 & 1 & 1.4 & 0.77 & 0.55 & 0.61
\end{tabular}

Figure 10: vast-2015-mc1-3d results normalized by splatt (serial)

$\begin{array}{lcccccc} & \text { qsort } & \text { splatt } & \text { 1-sadilla } & \text { 2-sadilla } & \text { quesadilla } & \text { radix } \\ 123 & 4.72 & 1 & 1.54 & 0.38 & 0 & 4.68 \\ 132 & 4.05 & 1 & 1.41 & 2.13 & 0.84 & 3.22 \\ 213 & 3.35 & 1 & 1.44 & 0.72 & 0.62 & 1.14 \\ 231 & 2.72 & 1 & 1.2 & 1.47 & 0.72 & 0.82 \\ 312 & 3.63 & 1 & 3.91 & 1.55 & 0.48 & 3.01 \\ 321 & 1.95 & 1 & 2.08 & 0.88 & 0.41 & 0.61\end{array}$


Figure 11: chicago-crime-comm results normalized by splatt (serial)

\begin{tabular}{|c|c|c|c|c|c|c|c|}
\hline & qsort & splatt & 1-sadilla & 2-sadilla & 3-sadilla & quesadilla & radix \\
\hline 1234 & 3.2 & 1 & 1.43 & 0.95 & 0.38 & 0 & 3.07 \\
\hline 1243 & 2.46 & 1 & 1.37 & 1.02 & 1.22 & 0.89 & 1.32 \\
\hline 1324 & 2.17 & 1 & 1.23 & 1.01 & 0.82 & 0.61 & 1.62 \\
\hline 1342 & 2.19 & 1 & 1.33 & 1.18 & 1.47 & 1.24 & 1.55 \\
\hline 1423 & 2.08 & 1 & 1.31 & 0.86 & 0.62 & 0.38 & 1.1 \\
\hline 1432 & 2.33 & 1 & 1.51 & 1.26 & 0.95 & 0.82 & 1.45 \\
\hline 2134 & 3.79 & 1 & 2.15 & 1.09 & 0.67 & 0.38 & 2.24 \\
\hline 2143 & 2.91 & 1 & 1.88 & 1.07 & 1.02 & 0.85 & 1.14 \\
\hline 2314 & 1.77 & 1 & 1.67 & 0.82 & 0.4 & 0.28 & 0.73 \\
\hline 2341 & 1.76 & 1 & 1.65 & 1.21 & 0.89 & 0.53 & 0.6 \\
\hline 2413 & 1.94 & 1 & 1.75 & 0.85 & 0.4 & 0.24 & 0.57 \\
\hline 2431 & 1.91 & 1 & 1.76 & 1.33 & 0.64 & 0.31 & 0.51 \\
\hline 3124 & 4.38 & 1 & 2.07 & 0.99 & 0.71 & 0.39 & 2.17 \\
\hline 3142 & 3.42 & 1 & 1.9 & 1.02 & 1.39 & 1.14 & 1.62 \\
\hline 3214 & 2.15 & 1 & 1.63 & 1.03 & 0.6 & 0.54 & 0.75 \\
\hline 3241 & 2.07 & 1 & 1.62 & 1.47 & 0.9 & 0.55 & 0.62 \\
\hline 3412 & 2.19 & 1 & 1.76 & 1.13 & 0.68 & 0.53 & 0.77 \\
\hline 3421 & 2.11 & 1 & 1.72 & 1.54 & 0.83 & 0.54 & 0.61 \\
\hline 4123 & 4.7 & 1 & 2.22 & 1.13 & 0.74 & 0.35 & 1.73 \\
\hline 4132 & 3.33 & 1 & 1.96 & 1.14 & 1.59 & 1.37 & 1.52 \\
\hline 4213 & 2.28 & 1 & 1.7 & 0.94 & 0.44 & 0.26 & 0.62 \\
\hline 4231 & 2.1 & 1 & 1.62 & 1.41 & 0.66 & 0.31 & 0.52 \\
\hline 4312 & 2.15 & 1 & 1.77 & 0.79 & 0.37 & 0.25 & 0.69 \\
\hline 4321 & 2.11 & 1 & 1.76 & 1.23 & 0.83 & 0.5 & 0.53 \\
\hline
\end{tabular}

Figure 12: delicious-4d results normalized by splatt (serial)

\begin{tabular}{|c|c|c|c|c|c|c|c|}
\hline & qsort & splatt & 1-sadilla & 2-sadilla & 3-sadilla & quesadilla & radix \\
\hline 1234 & 3.51 & 1 & 1.43 & 0.47 & 0.31 & 0 & 5.44 \\
\hline 1243 & 3.64 & 1 & 1.49 & 0.49 & 2.28 & 1.84 & 4.9 \\
\hline 1324 & 2.61 & 1 & 1.49 & 1.54 & 1.31 & 1.15 & 2.36 \\
\hline 1342 & 2.58 & 1 & 1.5 & 1.59 & 2.05 & 1.81 & 2.6 \\
\hline 1423 & 2.58 & 1 & 1.3 & 1.26 & 1.07 & 0.77 & 2.93 \\
\hline 1432 & 2.52 & 1 & 1.38 & 1.43 & 2.35 & 2.12 & 2.85 \\
\hline 2134 & 2.68 & 1 & 1.35 & 1.1 & 0.99 & 0.87 & 2.07 \\
\hline 2143 & 2.57 & 1 & 1.24 & 1.05 & 1.81 & 1.6 & 2.03 \\
\hline 2314 & 2.22 & 1 & 1.29 & 1.38 & 1.29 & 1.18 & 1.7 \\
\hline 2341 & 2.24 & 1 & 1.29 & 1.48 & 1.47 & 1.35 & 1.59 \\
\hline 2413 & 2.23 & 1 & 1.24 & 0.88 & 0.85 & 0.65 & 1.45 \\
\hline 2431 & 2.19 & 1 & 1.3 & 0.91 & 1.38 & 1.26 & 1.47 \\
\hline 3124 & 3.95 & 1 & 1.58 & 0.9 & 0.71 & 0.55 & 2.27 \\
\hline 3142 & 3.46 & 1 & 1.51 & 0.89 & 2.08 & 1.87 & 2.21 \\
\hline 3214 & 2.85 & 1 & 1.67 & 1.3 & 1.22 & 1.1 & 1.44 \\
\hline 3241 & 2.8 & 1 & 1.7 & 1.38 & 1.15 & 1.04 & 1.25 \\
\hline 3412 & 2.5 & 1 & 1.53 & 0.84 & 0.67 & 0.54 & 1.62 \\
\hline 3421 & 2.65 & 1 & 1.68 & 1.13 & 1.36 & 1.24 & 1.29 \\
\hline 4123 & 4.8 & 1 & 2.05 & 0.99 & 0.73 & 0.38 & 4.06 \\
\hline 4132 & 3.75 & 1 & 1.84 & 1 & 2.44 & 2.25 & 3.01 \\
\hline 4213 & 2.67 & 1 & 1.81 & 1.26 & 1.22 & 0.95 & 1.73 \\
\hline 4231 & 2.69 & 1 & 1.85 & 1.34 & 1.69 & 1.54 & 1.76 \\
\hline 4312 & 2.54 & 1 & 1.81 & 0.95 & 0.76 & 0.61 & 1.74 \\
\hline 4321 & 2.69 & 1 & 1.96 & 1.23 & 1.5 & 1.38 & 1.41 \\
\hline
\end{tabular}


Figure 13: enron results normalized by splatt (serial)

\begin{tabular}{l|c|c|c|c|c|c|c} 
& qsort & splatt & 1-sadilla & 2-sadilla & 3-sadilla & quesadilla & radix \\
1234 & 3.06 & 1 & 1.96 & 1.3 & 0.37 & 0 & 2.83 \\
1243 & 2.17 & 1 & 1.61 & 1.19 & 0.81 & 0.36 & 1.27 \\
1324 & 1.88 & 1 & 1.38 & 1.04 & 0.81 & 0.65 & 1.18 \\
1342 & 1.85 & 1 & 1.39 & 1.22 & 1.25 & 0.97 & 1.03 \\
1423 & 1.91 & 1 & 1.52 & 0.82 & 0.63 & 0.25 & 1.01 \\
1432 & 1.81 & 1 & 1.46 & 1.01 & 1.02 & 0.77 & 0.99 \\
2134 & 4.08 & 1 & 2.02 & 1.64 & 0.73 & 0.39 & 2.62 \\
2143 & 2.76 & 1 & 1.66 & 1.43 & 1.09 & 0.63 & 1.26 \\
2314 & 2.3 & 1 & 1.54 & 1.25 & 1.15 & 0.92 & 1.25 \\
2341 & 2.44 & 1 & 1.64 & 1.42 & 1.44 & 1.23 & 1.46 \\
2413 & 2.39 & 1 & 1.65 & 0.77 & 0.74 & 0.3 & 1.01 \\
2431 & 2.5 & 1 & 1.73 & 0.86 & 1.18 & 1.1 & 1.29 \\
3124 & 3.11 & 1 & 1.43 & 1.04 & 0.72 & 0.48 & 1.22 \\
3142 & 2.48 & 1 & 1.35 & 1.06 & 1.38 & 1.1 & 0.92 \\
3214 & 2.15 & 1 & 1.31 & 0.85 & 0.78 & 0.59 & 0.78 \\
3241 & 2.23 & 1 & 1.38 & 0.96 & 0.86 & 0.75 & 0.94 \\
3412 & 2.01 & 1 & 1.34 & 0.82 & 0.8 & 0.5 & 0.79 \\
3421 & 2.14 & 1 & 1.44 & 0.99 & 0.88 & 0.71 & 0.8 \\
4123 & 5.53 & 1 & 2.23 & 1.77 & 1.3 & 0.39 & 2.46 \\
4132 & 2.85 & 1 & 1.47 & 1.2 & 1.35 & 1.07 & 1.18 \\
4213 & 3.56 & 1 & 1.77 & 1.15 & 1.1 & 0.47 & 1.43 \\
4231 & 3.42 & 1 & 1.77 & 1.17 & 1.61 & 1.47 & 1.68 \\
4312 & 2.42 & 1 & 1.46 & 0.9 & 0.86 & 0.54 & 0.89 \\
4321 & 2.63 & 1 & 1.58 & 1.06 & 1.01 & 0.96 & 1.07
\end{tabular}

Figure 14: flickr-4d results normalized by splatt (serial)

\begin{tabular}{|c|c|c|c|c|c|c|c|}
\hline & qsort & splatt & 1-sadilla & 2-sadilla & 3-sadilla & quesadilla & radix \\
\hline 1234 & 3.45 & 1 & 1.47 & 0.47 & 0.28 & 0 & 4.93 \\
\hline 1243 & 3.51 & 1 & 1.54 & 0.48 & 1.44 & 0.9 & 3.49 \\
\hline 1324 & 2.44 & 1 & 1.4 & 1.27 & 0.99 & 0.89 & 1.61 \\
\hline 1342 & 2.29 & 1 & 1.31 & 1.22 & 1.51 & 1.23 & 1.64 \\
\hline 1423 & 3.33 & 1 & 1.47 & 1.76 & 1.18 & 0.78 & 4.45 \\
\hline 1432 & 2.34 & 1 & 1.28 & 1.44 & 1.83 & 1.53 & 2.08 \\
\hline 2134 & 4.21 & 1 & 1.28 & 1.32 & 1.12 & 0.92 & 2.53 \\
\hline 2143 & 3.96 & 1 & 1.26 & 1.26 & 1.55 & 1.17 & 2.51 \\
\hline 2314 & 4.01 & 1 & 1.3 & 2.74 & 2.74 & 2.56 & 3.13 \\
\hline 2341 & 4.22 & 1 & 1.39 & 3.07 & 3.44 & 3.06 & 3.31 \\
\hline 2413 & 3.9 & 1 & 1.22 & 1.15 & 1.21 & 0.85 & 2.02 \\
\hline 2431 & 4.01 & 1 & 1.34 & 1.26 & 2.08 & 1.86 & 2.11 \\
\hline 3124 & 4.63 & 1 & 1.72 & 1.19 & 0.79 & 0.61 & 2.02 \\
\hline 3142 & 4.49 & 1 & 1.66 & 1.19 & 2.16 & 1.71 & 2.14 \\
\hline 3214 & 3.66 & 1 & 1.75 & 1.31 & 1.36 & 1.19 & 1.59 \\
\hline 3241 & 3.61 & 1 & 1.73 & 1.33 & 1.28 & 1.16 & 1.37 \\
\hline 3412 & 3.06 & 1 & 1.54 & 1.03 & 0.94 & 0.62 & 1.51 \\
\hline 3421 & 3.19 & 1 & 1.62 & 1.17 & 1.36 & 1.15 & 1.28 \\
\hline 4123 & 4.68 & 1 & 2.3 & 1.27 & 0.78 & 0.38 & 3.93 \\
\hline 4132 & 3.17 & 1 & 1.79 & 1.12 & 1.68 & 1.37 & 1.79 \\
\hline 4213 & 2.72 & 1 & 1.96 & 0.97 & 0.91 & 0.73 & 1.49 \\
\hline 4231 & 2.77 & 1 & 1.95 & 0.96 & 1.96 & 1.85 & 2.01 \\
\hline 4312 & 2.36 & 1 & 1.77 & 0.85 & 0.77 & 0.53 & 1.24 \\
\hline 4321 & 2.49 & 1 & 1.85 & 0.99 & 1.14 & 1.07 & 1.12 \\
\hline
\end{tabular}


Figure 15: nips results normalized by splatt (serial)

\begin{tabular}{|c|c|c|c|c|c|c|c|}
\hline & qsort & splatt & 1-sadilla & 2-sadilla & 3-sadilla & quesadilla & radix \\
\hline 1234 & 3.88 & 1 & 1.89 & 1.65 & 0.34 & 0 & 3.78 \\
\hline 1243 & 3.85 & 1 & 1.9 & 1.66 & 2.41 & 0.78 & 3.48 \\
\hline 1324 & 0.85 & 1 & 0.44 & 0.76 & 0.73 & 0.66 & 0.88 \\
\hline 1342 & 0.86 & 1 & 0.44 & 0.76 & 0.92 & 0.82 & 0.88 \\
\hline 1423 & 3.79 & 1 & 1.88 & 2.6 & 2.37 & 0.77 & 3.52 \\
\hline 1432 & 0.61 & 1 & 0.31 & 0.41 & 0.6 & 0.53 & 0.6 \\
\hline 2134 & 5.67 & 1 & 2.49 & 2.21 & 0.96 & 0.62 & 4.01 \\
\hline 2143 & 5.61 & 1 & 2.48 & 2.2 & 2.7 & 1.15 & 3.3 \\
\hline 2314 & 3.68 & 1 & 1.89 & 2.05 & 2.04 & 1.6 & 2.53 \\
\hline 2341 & 3.66 & 1 & 1.88 & 2.06 & 2.54 & 2.29 & 2.2 \\
\hline 2413 & 5.49 & 1 & 2.42 & 2.51 & 2.44 & 0.91 & 3.33 \\
\hline 2431 & 4.36 & 1 & 2.03 & 1.99 & 2.59 & 2.32 & 2.98 \\
\hline 3124 & 2.89 & 1 & 1.35 & 0.82 & 0.73 & 0.57 & 1.25 \\
\hline 3142 & 2.95 & 1 & 1.4 & 0.85 & 1.23 & 0.97 & 1.29 \\
\hline 3214 & 2.42 & 1 & 1.58 & 0.86 & 0.84 & 0.71 & 0.92 \\
\hline 3241 & 2.32 & 1 & 1.57 & 0.78 & 0.94 & 0.81 & 0.91 \\
\hline 3412 & 2.79 & 1 & 1.28 & 1.34 & 1.09 & 0.87 & 1.17 \\
\hline 3421 & 2.37 & 1 & 1.41 & 1.47 & 0.98 & 0.87 & 0.99 \\
\hline 4123 & 3.44 & 1 & 3.14 & 2.15 & 1.89 & 0.51 & 3.18 \\
\hline 4132 & 1.82 & 1 & 1.73 & 1.15 & 1.86 & 1.64 & 1.83 \\
\hline 4213 & 2.55 & 1 & 2.41 & 1.36 & 1.28 & 0.48 & 1.75 \\
\hline 4231 & 2.46 & 1 & 2.33 & 1.32 & 1.73 & 1.55 & 1.97 \\
\hline 4312 & 1.88 & 1 & 1.73 & 0.85 & 0.67 & 0.5 & 0.88 \\
\hline 4321 & 2.06 & 1 & 2 & 1.27 & 0.96 & 0.85 & 0.96 \\
\hline
\end{tabular}

Figure 16: uber results normalized by splatt (serial)

\begin{tabular}{|c|c|c|c|c|c|c|c|}
\hline & qsort & splatt & 1-sadilla & 2-sadilla & 3-sadilla & quesadilla & radix \\
\hline 1234 & 3.41 & 1 & 2.19 & 1.5 & 0.62 & 0 & 2.83 \\
\hline 1243 & 2.27 & 1 & 1.68 & 1.34 & 1.08 & 0.79 & 1.46 \\
\hline 1324 & 1.95 & 1 & 1.44 & 1.06 & 0.79 & 0.46 & 1.5 \\
\hline 1342 & 2.06 & 1 & 1.47 & 1.36 & 1.35 & 1.14 & 1.69 \\
\hline 1423 & 1.95 & 1 & 1.52 & 0.77 & 0.6 & 0.34 & 1.12 \\
\hline 1432 & 2.12 & 1 & 1.66 & 1.13 & 1.11 & 0.91 & 1.4 \\
\hline 2134 & 4.36 & 1 & 2.79 & 1.85 & 1.03 & 0.46 & 2.54 \\
\hline 2143 & 2.82 & 1 & 2.06 & 1.54 & 1.5 & 1.2 & 1.44 \\
\hline 2314 & 1.93 & 1 & 1.73 & 1.01 & 0.63 & 0.37 & 1 \\
\hline 2341 & 1.88 & 1 & 1.74 & 1.39 & 1.01 & 0.71 & 1.08 \\
\hline 2413 & 1.94 & 1 & 1.78 & 0.77 & 0.48 & 0.26 & 0.75 \\
\hline 2431 & 2.07 & 1 & 1.88 & 1.21 & 0.87 & 0.61 & 0.92 \\
\hline 3124 & 3.96 & 1 & 2.02 & 1.33 & 0.88 & 0.46 & 2.25 \\
\hline 3142 & 2.7 & 1 & 1.76 & 1.27 & 1.48 & 1.38 & 1.74 \\
\hline 3214 & 2.22 & 1 & 1.56 & 1.24 & 0.89 & 0.64 & 0.85 \\
\hline 3241 & 2.06 & 1 & 1.55 & 1.57 & 0.97 & 0.68 & 1.02 \\
\hline 3412 & 1.9 & 1 & 1.59 & 1 & 0.72 & 0.55 & 0.97 \\
\hline 3421 & 1.98 & 1 & 1.67 & 1.37 & 1.1 & 0.74 & 0.92 \\
\hline 4123 & 4.82 & 1 & 2.04 & 1.34 & 0.93 & 0.46 & 2.3 \\
\hline 4132 & 3.13 & 1 & 1.74 & 1.26 & 1.61 & 1.35 & 1.75 \\
\hline 4213 & 2.6 & 1 & 1.61 & 1.26 & 0.9 & 0.65 & 0.85 \\
\hline 4231 & 2.44 & 1 & 1.54 & 1.55 & 0.96 & 0.6 & 1.01 \\
\hline 4312 & 2.26 & 1 & 1.61 & 0.99 & 0.72 & 0.55 & 0.96 \\
\hline 4321 & 2.34 & 1 & 1.65 & 1.34 & 1.08 & 0.82 & 0.91 \\
\hline
\end{tabular}


Figure 17: lbnl-network results normalized by splatt (serial) (1)

\begin{tabular}{|c|c|c|c|c|c|c|c|c|}
\hline & qsort & splatt & 1-sadilla & 2-sadilla & 3-sadilla & 4-sadilla & quesadilla & radix \\
\hline 12345 & 2.01 & 1 & 1.42 & 1.33 & 1.21 & 1.14 & 0 & 1.46 \\
\hline 12354 & 1.76 & 1 & 1.25 & 1.15 & 1.07 & 0.84 & 0.7 & 1.34 \\
\hline 12435 & 1.65 & 1 & 1.14 & 1.08 & 1.26 & 1.24 & 0.4 & 1 \\
\hline 12453 & 1.69 & 1 & 1.21 & 1.13 & 1.35 & 1.07 & 0.95 & 1.19 \\
\hline 12534 & 2.12 & 1 & 1.5 & 1.4 & 0.93 & 0.87 & 0.7 & 1.48 \\
\hline 12543 & 2.08 & 1 & 1.52 & 1.4 & 0.94 & 1.33 & 1.18 & 1.52 \\
\hline 13245 & 1.46 & 1 & 1 & 1.21 & 1.16 & 1.09 & 0.33 & 0.97 \\
\hline 13254 & 1.46 & 1 & 1.04 & 1.25 & 1.19 & 0.9 & 0.8 & 0.95 \\
\hline 13425 & 1.44 & 1 & 0.99 & 1.2 & 1.37 & 1.32 & 0.58 & 0.96 \\
\hline 13452 & 1.41 & 1 & 1.02 & 1.23 & 1.42 & 1.13 & 1.02 & 1.05 \\
\hline 13524 & 1.84 & 1 & 1.31 & 1.61 & 1.04 & 1.05 & 0.99 & 1.35 \\
\hline 13542 & 1.88 & 1 & 1.3 & 1.61 & 1.12 & 1.46 & 1.33 & 1.25 \\
\hline 14235 & 1.51 & 1 & 1.06 & 1.19 & 1.12 & 1.11 & 0.35 & 0.89 \\
\hline 14253 & 1.58 & 1 & 1.15 & 1.26 & 1.21 & 1 & 0.8 & 1.07 \\
\hline 14325 & 1.49 & 1 & 1.04 & 1.17 & 1.31 & 1.28 & 0.56 & 0.93 \\
\hline 14352 & 1.47 & 1 & 1.06 & 1.18 & 1.37 & 1.09 & 0.99 & 1.01 \\
\hline 14523 & 1.93 & 1 & 1.39 & 1.51 & 1.14 & 1.14 & 1 & 1.33 \\
\hline 14532 & 1.95 & 1 & 1.38 & 1.53 & 1.13 & 1.45 & 1.31 & 1.28 \\
\hline 15234 & 2.28 & 1 & 1.66 & 0.95 & 0.95 & 0.95 & 0.79 & 1.59 \\
\hline 15243 & 2.27 & 1 & 1.63 & 0.92 & 0.94 & 1.44 & 1.28 & 1.58 \\
\hline 15324 & 2.25 & 1 & 1.63 & 0.95 & 1.31 & 1.29 & 1.14 & 1.53 \\
\hline 15342 & 2.25 & 1 & 1.63 & 0.95 & 1.31 & 1.7 & 1.53 & 1.53 \\
\hline 15423 & 2.26 & 1 & 1.64 & 0.95 & 1.35 & 1.36 & 1.15 & 1.58 \\
\hline 15432 & 2.26 & 1 & 1.62 & 0.95 & 1.34 & 1.69 & 1.54 & 1.57 \\
\hline
\end{tabular}

Figure 18: lbnl-network results normalized by splatt (serial) (2)

\begin{tabular}{|c|c|c|c|c|c|c|c|c|}
\hline & qsort & splatt & 1-sadilla & 2-sadilla & 3-sadilla & 4-sadilla & quesadilla & radix \\
\hline 21345 & 2.58 & 1 & 1.85 & 1.64 & 1.53 & 1.43 & 0.35 & 1.41 \\
\hline 21354 & 2.17 & 1 & 1.59 & 1.41 & 1.32 & 1.04 & 0.9 & 1.25 \\
\hline 21435 & 2.21 & 1 & 1.56 & 1.41 & 1.47 & 1.47 & 0.58 & 1.14 \\
\hline 21453 & 2.28 & 1 & 1.67 & 1.49 & 1.57 & 1.36 & 1.23 & 1.24 \\
\hline 21534 & 2.67 & 1 & 1.97 & 1.74 & 1.21 & 1.21 & 1.05 & 1.44 \\
\hline 21543 & 2.67 & 1 & 1.95 & 1.74 & 1.13 & 1.44 & 1.42 & 1.48 \\
\hline 23145 & 1.81 & 1 & 1.43 & 1.21 & 1.18 & 1.11 & 0.37 & 0.95 \\
\hline 23154 & 1.75 & 1 & 1.42 & 1.23 & 1.21 & 0.97 & 0.86 & 1 \\
\hline 23415 & 1.72 & 1 & 1.34 & 1.18 & 1.24 & 1.23 & 0.51 & 0.92 \\
\hline 23451 & 1.79 & 1 & 1.45 & 1.25 & 1.33 & 0.95 & 0.84 & 0.95 \\
\hline 23514 & 2.12 & 1 & 1.72 & 1.46 & 0.88 & 0.87 & 0.81 & 1.2 \\
\hline 23541 & 2.13 & 1 & 1.72 & 1.5 & 0.95 & 1.2 & 1.08 & 1.24 \\
\hline 24135 & 1.74 & 1 & 1.4 & 1.15 & 1.12 & 1.08 & 0.37 & 0.92 \\
\hline 24153 & 1.91 & 1 & 1.5 & 1.23 & 1.18 & 1.05 & 0.94 & 1.01 \\
\hline 24315 & 1.71 & 1 & 1.38 & 1.16 & 1.21 & 1.2 & 0.49 & 0.89 \\
\hline 24351 & 1.85 & 1 & 1.45 & 1.24 & 1.3 & 0.93 & 0.83 & 0.88 \\
\hline 24513 & 2.25 & 1 & 1.81 & 1.54 & 0.99 & 0.99 & 0.86 & 1.22 \\
\hline 24531 & 2.26 & 1 & 1.82 & 1.53 & 0.99 & 1.18 & 1.05 & 1.21 \\
\hline 25134 & 2.56 & 1 & 2.06 & 0.9 & 0.9 & 0.9 & 0.75 & 1.41 \\
\hline 25143 & 2.61 & 1 & 2.09 & 0.91 & 0.9 & 1.38 & 1.16 & 1.38 \\
\hline 25314 & 2.56 & 1 & 2.13 & 0.91 & 1.12 & 1.13 & 0.98 & 1.4 \\
\hline 25341 & 2.57 & 1 & 2.12 & 0.91 & 1.12 & 1.36 & 1.21 & 1.41 \\
\hline 25413 & 2.56 & 1 & 2.1 & 0.9 & 1.2 & 1.2 & 1.04 & 1.45 \\
\hline 25431 & 2.65 & 1 & 2.07 & 0.89 & 1.19 & 1.41 & 1.24 & 1.45 \\
\hline
\end{tabular}


Figure 19: lbnl-network results normalized by splatt (serial) (3)

\begin{tabular}{|c|c|c|c|c|c|c|c|c|}
\hline & qsort & splatt & 1-sadilla & 2-sadilla & 3-sadilla & 4-sadilla & quesadilla & radix \\
\hline 31245 & 2.68 & 1 & 1.74 & 1.62 & 1.55 & 1.44 & 0.36 & 1.35 \\
\hline 31254 & 2.28 & 1 & 1.52 & 1.42 & 1.34 & 0.99 & 0.86 & 1.29 \\
\hline 31425 & 2.05 & 1 & 1.35 & 1.26 & 1.46 & 1.41 & 0.56 & 1.08 \\
\hline 31452 & 2.2 & 1 & 1.44 & 1.33 & 1.58 & 1.26 & 1.13 & 1.23 \\
\hline 31524 & 2.67 & 1 & 1.81 & 1.66 & 1.15 & 1.15 & 1 & 1.53 \\
\hline 31542 & 2.7 & 1 & 1.83 & 1.66 & 1.16 & 1.43 & 1.26 & 1.52 \\
\hline 32145 & 2.06 & 1 & 1.46 & 1.42 & 1.35 & 1.29 & 0.43 & 1.09 \\
\hline 32154 & 1.9 & 1 & 1.32 & 1.29 & 1.25 & 0.97 & 0.86 & 1.06 \\
\hline 32415 & 1.79 & 1 & 1.28 & 1.23 & 1.31 & 1.3 & 0.54 & 0.96 \\
\hline 32451 & 1.98 & 1 & 1.37 & 1.33 & 1.41 & 0.93 & 0.89 & 1.06 \\
\hline 32514 & 2.31 & 1 & 1.66 & 1.59 & 0.93 & 0.93 & 0.87 & 1.24 \\
\hline 32541 & 2.34 & 1 & 1.64 & 1.6 & 1 & 1.27 & 1.14 & 1.2 \\
\hline 34125 & 1.84 & 1 & 1.26 & 1.24 & 1.17 & 1.13 & 0.39 & 0.96 \\
\hline 34152 & 1.91 & 1 & 1.33 & 1.35 & 1.28 & 1.01 & 0.9 & 1.09 \\
\hline 34215 & 1.66 & 1 & 1.19 & 1.23 & 1.21 & 1.2 & 0.5 & 0.89 \\
\hline 34251 & 1.79 & 1 & 1.29 & 1.29 & 1.27 & 0.89 & 0.83 & 0.98 \\
\hline 34512 & 2.34 & 1 & 1.65 & 1.71 & 1.03 & 1.03 & 0.89 & 1.28 \\
\hline 34521 & 2.32 & 1 & 1.66 & 1.73 & 1.02 & 1.28 & 1.14 & 1.3 \\
\hline 35124 & 2.79 & 1 & 1.96 & 0.96 & 0.97 & 0.96 & 0.81 & 1.54 \\
\hline 35142 & 2.72 & 1 & 1.98 & 0.97 & 0.97 & 1.43 & 1.27 & 1.51 \\
\hline 35214 & 2.69 & 1 & 1.97 & 0.9 & 1.21 & 1.27 & 1.11 & 1.57 \\
\hline 35241 & 2.77 & 1 & 2 & 0.97 & 1.26 & 1.52 & 1.36 & 1.42 \\
\hline 35412 & 2.81 & 1 & 1.97 & 0.97 & 1.28 & 1.28 & 1.13 & 1.43 \\
\hline 35421 & 2.74 & 1 & 1.96 & 0.9 & 1.27 & 1.5 & 1.35 & 1.56 \\
\hline
\end{tabular}

Figure 20: lbnl-network results normalized by splatt (serial) (4)

\begin{tabular}{|c|c|c|c|c|c|c|c|c|}
\hline & qsort & splatt & 1-sadilla & 2-sadilla & 3-sadilla & 4-sadilla & quesadilla & radix \\
\hline 41235 & 2.82 & 1 & 1.88 & 1.62 & 1.51 & 1.48 & 0.36 & 1.44 \\
\hline 41253 & 2.86 & 1 & 1.9 & 1.66 & 1.54 & 1.34 & 1.11 & 1.53 \\
\hline 41325 & 2.29 & 1 & 1.56 & 1.33 & 1.58 & 1.54 & 0.62 & 1.15 \\
\hline 41352 & 2.31 & 1 & 1.6 & 1.4 & 1.69 & 1.4 & 1.28 & 1.28 \\
\hline 41523 & 2.99 & 1 & 2.03 & 1.76 & 1.38 & 1.36 & 1.21 & 1.65 \\
\hline 41532 & 3 & 1 & 2.05 & 1.79 & 1.38 & 1.84 & 1.66 & 1.59 \\
\hline 42135 & 1.81 & 1 & 1.37 & 1.15 & 1.1 & 1.1 & 0.37 & 0.91 \\
\hline 42153 & 1.96 & 1 & 1.51 & 1.23 & 1.19 & 1.04 & 0.94 & 1.01 \\
\hline 42315 & 1.79 & 1 & 1.36 & 1.16 & 1.22 & 1.22 & 0.5 & 0.89 \\
\hline 42351 & 1.86 & 1 & 1.48 & 1.26 & 1.32 & 0.94 & 0.84 & 1 \\
\hline 42513 & 2.41 & 1 & 1.89 & 1.6 & 1.03 & 1.03 & 0.9 & 1.28 \\
\hline 42531 & 2.44 & 1 & 1.93 & 1.6 & 1.02 & 1.23 & 1.11 & 1.28 \\
\hline 43125 & 1.68 & 1 & 1.33 & 1.13 & 1.07 & 1.04 & 0.35 & 0.87 \\
\hline 43152 & 1.78 & 1 & 1.4 & 1.19 & 1.14 & 0.95 & 0.85 & 0.97 \\
\hline 43215 & 1.74 & 1 & 1.36 & 1.24 & 1.18 & 1.22 & 0.51 & 0.91 \\
\hline 43251 & 1.91 & 1 & 1.5 & 1.35 & 1.35 & 0.88 & 0.8 & 1 \\
\hline 43512 & 2.25 & 1 & 1.74 & 1.6 & 0.96 & 0.96 & 0.83 & 1.2 \\
\hline 43521 & 2.25 & 1 & 1.73 & 1.57 & 0.95 & 1.19 & 1.07 & 1.23 \\
\hline 45123 & 2.82 & 1 & 2.17 & 0.97 & 0.92 & 0.97 & 0.82 & 1.42 \\
\hline 45132 & 2.75 & 1 & 2.2 & 0.97 & 0.97 & 1.46 & 1.31 & 1.49 \\
\hline 45213 & 2.76 & 1 & 2.21 & 0.94 & 1.16 & 1.28 & 1.1 & 1.55 \\
\hline 45231 & 2.83 & 1 & 2.18 & 0.98 & 1.27 & 1.5 & 1.34 & 1.54 \\
\hline 45312 & 2.86 & 1 & 2.16 & 0.98 & 1.22 & 1.21 & 1.06 & 1.51 \\
\hline 45321 & 2.76 & 1 & 2.17 & 0.97 & 1.21 & 1.41 & 1.26 & 1.49 \\
\hline
\end{tabular}


Figure 21: lbnl-network results normalized by splatt (serial) (5)

\begin{tabular}{|c|c|c|c|c|c|c|c|c} 
& qsort & splatt & 1-sadilla & 2-sadilla & 3-sadilla & 4-sadilla & quesadilla & radix \\
51234 & 6.09 & 1 & 1.77 & 1.66 & 1.65 & 1.66 & 1.26 & 3.29 \\
51243 & 6.04 & 1 & 1.67 & 1.58 & 1.66 & 3.16 & 2.67 & 3.27 \\
51324 & 6.05 & 1 & 1.75 & 1.64 & 3.05 & 3.02 & 2.57 & 3.2 \\
51342 & 6.29 & 1 & 1.86 & 1.73 & 3.23 & 4.63 & 4.19 & 3.06 \\
51423 & 6.06 & 1 & 1.76 & 1.64 & 3.11 & 3.11 & 2.63 & 3.19 \\
51432 & 6.36 & 1 & 1.87 & 1.74 & 3 & 4.39 & 4.2 & 3.4 \\
52134 & 6.06 & 1 & 1.76 & 2.26 & 2.24 & 2.26 & 1.87 & 3.29 \\
52143 & 6.04 & 1 & 1.78 & 2.28 & 2.27 & 3.67 & 3.3 & 3.34 \\
52314 & 5.92 & 1 & 1.79 & 2.28 & 2.75 & 2.75 & 2.34 & 3.31 \\
52341 & 5.97 & 1 & 1.78 & 2.27 & 2.75 & 3.28 & 2.86 & 3.28 \\
52413 & 5.99 & 1 & 1.76 & 2.25 & 2.77 & 2.77 & 2.38 & 3.27 \\
52431 & 6.02 & 1 & 1.77 & 2.27 & 2.83 & 3.26 & 2.88 & 3.3 \\
53124 & 6.2 & 1 & 1.83 & 2.22 & 2.2 & 2.21 & 1.8 & 3.33 \\
53142 & 6.27 & 1 & 1.86 & 2.21 & 2.23 & 3.66 & 3.27 & 3.31 \\
53214 & 6.29 & 1 & 1.84 & 2.01 & 2.5 & 2.7 & 2.29 & 3.05 \\
53241 & 6.27 & 1 & 1.85 & 2.21 & 2.72 & 3.27 & 2.85 & 2.98 \\
53412 & 6.22 & 1 & 1.83 & 2.19 & 2.75 & 2.61 & 2.33 & 3.31 \\
53421 & 6.11 & 1 & 1.84 & 2.2 & 2.75 & 3.24 & 2.89 & 3.33 \\
54123 & 6.21 & 1 & 1.82 & 2.31 & 2.32 & 2.16 & 1.79 & 3.41 \\
54132 & 6.09 & 1 & 1.84 & 2.35 & 2.35 & 3.7 & 3.29 & 3.38 \\
54213 & 6.15 & 1 & 1.82 & 2.34 & 2.84 & 2.82 & 2.46 & 3.35 \\
54231 & 6.2 & 1 & 1.86 & 2.39 & 2.9 & 3.4 & 3.01 & 3.44 \\
54312 & 6.28 & 1 & 1.7 & 2.36 & 2.83 & 2.81 & 2.43 & 3.42 \\
54321 & 6.02 & 1 & 1.74 & 2.33 & 2.78 & 3.14 & 2.91 & 3.37
\end{tabular}

Figure 22: vast-2015-mc1-5d results normalized by splatt (serial) (1)

\begin{tabular}{|c|c|c|c|c|c|c|c|c|}
\hline & qsort & splatt & 1-sadilla & 2-sadilla & 3-sadilla & 4-sadilla & quesadilla & radix \\
\hline 12345 & 3.38 & 1 & 1.07 & 0.22 & 0.22 & 0.22 & 0 & 4.24 \\
\hline 12354 & 3.41 & 1 & 1.1 & 0.23 & 0.23 & 2.99 & 2.63 & 4.13 \\
\hline 12435 & 3.39 & 1 & 1.08 & 0.23 & 2.99 & 2.76 & 2.79 & 4.44 \\
\hline 12453 & 3.35 & 1 & 1.07 & 0.22 & 2.86 & 6.36 & 5.57 & 4.98 \\
\hline 12534 & 3.36 & 1 & 1.08 & 0.23 & 2.82 & 3.01 & 2.72 & 4.39 \\
\hline 12543 & 3.41 & 1 & 1.08 & 0.23 & 3.09 & 5.96 & 5.78 & 4.75 \\
\hline 13245 & 2.82 & 1 & 0.92 & 1.44 & 0.81 & 0.76 & 0.62 & 3.56 \\
\hline 13254 & 2.81 & 1 & 0.93 & 1.49 & 0.81 & 3.24 & 3.16 & 3.56 \\
\hline 13425 & 2.11 & 1 & 0.97 & 1.28 & 1.35 & 1.3 & 1.12 & 2.23 \\
\hline 13452 & 2.01 & 1 & 0.98 & 1.25 & 1.41 & 2.29 & 2.21 & 1.9 \\
\hline 13524 & 2.19 & 1 & 0.98 & 1.3 & 1.31 & 1.33 & 1.08 & 2.28 \\
\hline 13542 & 2.1 & 1 & 0.96 & 1.22 & 1.4 & 2.3 & 2.18 & 1.78 \\
\hline 14235 & 2.26 & 1 & 1.05 & 1.29 & 1.08 & 1.17 & 0.98 & 2.32 \\
\hline 14253 & 2.37 & 1 & 1.06 & 1.26 & 1.14 & 2.83 & 2.59 & 2.84 \\
\hline 14325 & 2.32 & 1 & 1.04 & 1.28 & 1.78 & 1.83 & 1.51 & 2.28 \\
\hline 14352 & 2.2 & 1 & 1.02 & 1.21 & 1.91 & 2.56 & 2.29 & 2.09 \\
\hline 14523 & 2.19 & 1 & 1.02 & 1.3 & 2.3 & 2.27 & 2 & 2.36 \\
\hline 14532 & 2.19 & 1 & 1.01 & 1.29 & 2.24 & 2.94 & 2.63 & 1.9 \\
\hline 15234 & 2.38 & 1 & 1.06 & 1.29 & 1.16 & 1.18 & 0.98 & 2.46 \\
\hline 15243 & 2.4 & 1 & 1.08 & 1.31 & 1.11 & 2.59 & 2.73 & 2.88 \\
\hline 15324 & 2.26 & 1 & 1.04 & 1.29 & 1.86 & 1.86 & 1.63 & 2.46 \\
\hline 15342 & 2.19 & 1 & 1.02 & 1.21 & 1.92 & 2.48 & 2.31 & 2.11 \\
\hline 15423 & 2.22 & 1 & 1.02 & 1.31 & 2.39 & 2.28 & 2.16 & 2.15 \\
\hline 15432 & 2.23 & 1 & 1.02 & 1.31 & 2.32 & 2.84 & 2.65 & 2.15 \\
\hline
\end{tabular}


Figure 23: vast-2015-mc1-5d results normalized by splatt (serial) (2)

\begin{tabular}{|c|c|c|c|c|c|c|c|c|}
\hline & qsort & splatt & 1-sadilla & 2-sadilla & 3-sadilla & 4-sadilla & quesadilla & radix \\
\hline 21345 & 2.32 & 1 & 0.99 & 0.49 & 0.53 & 0.52 & 0.44 & 1.54 \\
\hline 21354 & 2.34 & 1 & 0.96 & 0.5 & 0.52 & 1.78 & 1.65 & 1.46 \\
\hline 21435 & 2.43 & 1 & 0.97 & 0.54 & 1.74 & 1.86 & 1.67 & 1.5 \\
\hline 21453 & 2.3 & 1 & 0.99 & 0.51 & 1.71 & 2.93 & 3.03 & 1.83 \\
\hline 21534 & 2.23 & 1 & 0.98 & 0.51 & 1.67 & 1.82 & 1.66 & 1.45 \\
\hline 21543 & 2.41 & 1 & 0.99 & 0.54 & 1.79 & 3.02 & 3.15 & 1.73 \\
\hline 23145 & 1.95 & 1 & 0.81 & 1.03 & 0.61 & 0.63 & 0.59 & 1.17 \\
\hline 23154 & 1.93 & 1 & 0.8 & 1.03 & 0.59 & 1.65 & 1.51 & 1.24 \\
\hline 23415 & 1.52 & 1 & 0.91 & 1.05 & 0.65 & 0.56 & 0.51 & 1.09 \\
\hline 23451 & 1.52 & 1 & 0.91 & 1.01 & 0.79 & 0.67 & 0.57 & 0.71 \\
\hline 23514 & 1.48 & 1 & 0.85 & 0.98 & 0.65 & 0.56 & 0.47 & 1 \\
\hline 23541 & 1.48 & 1 & 0.88 & 0.98 & 0.79 & 0.68 & 0.55 & 0.71 \\
\hline 24135 & 1.58 & 1 & 0.97 & 0.69 & 0.53 & 0.54 & 0.47 & 1.16 \\
\hline 24153 & 1.67 & 1 & 0.96 & 0.69 & 0.53 & 1.38 & 1.24 & 1.28 \\
\hline 24315 & 1.51 & 1 & 0.93 & 0.65 & 0.9 & 0.76 & 0.69 & 1.14 \\
\hline 24351 & 1.52 & 1 & 0.95 & 0.77 & 0.95 & 0.67 & 0.56 & 0.81 \\
\hline 24513 & 1.52 & 1 & 0.96 & 0.77 & 0.65 & 0.61 & 0.55 & 0.77 \\
\hline 24531 & 1.51 & 1 & 0.97 & 0.78 & 0.61 & 0.81 & 0.78 & 0.82 \\
\hline 25134 & 1.56 & 1 & 0.93 & 0.65 & 0.55 & 0.55 & 0.49 & 1.08 \\
\hline 25143 & 1.67 & 1 & 0.95 & 0.69 & 0.52 & 1.33 & 1.18 & 1.38 \\
\hline 25314 & 1.52 & 1 & 0.91 & 0.68 & 0.93 & 0.72 & 0.7 & 1.04 \\
\hline 25341 & 1.5 & 1 & 0.92 & 0.76 & 0.95 & 0.68 & 0.56 & 0.82 \\
\hline 25413 & 1.52 & 1 & 0.92 & 0.82 & 0.65 & 0.61 & 0.5 & 0.84 \\
\hline 25431 & 1.56 & 1 & 0.91 & 0.8 & 0.62 & 0.8 & 0.7 & 0.83 \\
\hline
\end{tabular}

Figure 24: vast-2015-mc1-5d results normalized by splatt (serial) (3)

\begin{tabular}{|c|c|c|c|c|c|c|c|c|}
\hline & qsort & splatt & 1-sadilla & 2-sadilla & 3-sadilla & 4-sadilla & quesadilla & radix \\
\hline 31245 & 2.59 & 1 & 2.88 & 1.15 & 0.54 & 0.54 & 0.38 & 2.94 \\
\hline 31254 & 2.64 & 1 & 2.86 & 1.14 & 0.55 & 2.91 & 2.76 & 3.22 \\
\hline 31425 & 1.79 & 1 & 1.95 & 0.96 & 1.3 & 1.23 & 1.15 & 1.77 \\
\hline 31452 & 1.74 & 1 & 1.87 & 0.96 & 1.32 & 2.1 & 1.91 & 1.5 \\
\hline 31524 & 1.81 & 1 & 1.89 & 0.95 & 1.36 & 1.23 & 1.13 & 1.86 \\
\hline 31542 & 1.72 & 1 & 1.86 & 0.93 & 1.29 & 2.03 & 1.91 & 1.61 \\
\hline 32145 & 1.42 & 1 & 1.49 & 0.67 & 0.38 & 0.39 & 0.31 & 0.94 \\
\hline 32154 & 1.48 & 1 & 1.47 & 0.65 & 0.37 & 1.14 & 0.99 & 0.84 \\
\hline 32415 & 1.34 & 1 & 1.42 & 0.87 & 0.61 & 0.51 & 0.43 & 1 \\
\hline 32451 & 1.39 & 1 & 1.39 & 0.87 & 0.74 & 0.61 & 0.52 & 0.67 \\
\hline 32514 & 1.35 & 1 & 1.41 & 0.84 & 0.63 & 0.47 & 0.47 & 0.94 \\
\hline 32541 & 1.36 & 1 & 1.38 & 0.81 & 0.73 & 0.6 & 0.47 & 0.64 \\
\hline 34125 & 1.48 & 1 & 1.5 & 0.68 & 0.28 & 0.24 & 0.19 & 0.95 \\
\hline 34152 & 1.42 & 1 & 1.51 & 0.71 & 0.31 & 0.62 & 0.53 & 1.02 \\
\hline 34215 & 1.47 & 1 & 1.58 & 1.1 & 0.63 & 0.52 & 0.5 & 0.89 \\
\hline 34251 & 1.47 & 1 & 1.47 & 1.06 & 0.72 & 0.63 & 0.57 & 0.7 \\
\hline 34512 & 1.25 & 1 & 1.36 & 0.95 & 0.56 & 0.28 & 0.21 & 0.74 \\
\hline 34521 & 1.41 & 1 & 1.44 & 1.06 & 0.86 & 0.58 & 0.49 & 0.62 \\
\hline 35124 & 1.47 & 1 & 1.51 & 0.67 & 0.28 & 0.23 & 0.18 & 0.98 \\
\hline 35142 & 1.38 & 1 & 1.49 & 0.7 & 0.31 & 0.56 & 0.5 & 1.01 \\
\hline 35214 & 1.46 & 1 & 1.57 & 1.12 & 0.61 & 0.53 & 0.5 & 0.91 \\
\hline 35241 & 1.49 & 1 & 1.54 & 1.07 & 0.73 & 0.65 & 0.6 & 0.72 \\
\hline 35412 & 1.28 & 1 & 1.34 & 0.94 & 0.56 & 0.28 & 0.2 & 0.74 \\
\hline 35421 & 1.38 & 1 & 1.42 & 1.06 & 0.87 & 0.57 & 0.45 & 0.62 \\
\hline
\end{tabular}


Figure 25: vast-2015-mc1-5d results normalized by splatt (serial) (4)

\begin{tabular}{|c|c|c|c|c|c|c|c|c|}
\hline & qsort & splatt & 1-sadilla & 2-sadilla & 3-sadilla & 4-sadilla & quesadilla & radix \\
\hline 41235 & 5.3 & 1 & 2.28 & 0.86 & 0.66 & 0.68 & 0.47 & 3.38 \\
\hline 41253 & 5.02 & 1 & 2.22 & 0.83 & 0.65 & 1.98 & 1.74 & 4.15 \\
\hline 41325 & 4.9 & 1 & 2.15 & 0.8 & 1.16 & 0.98 & 0.84 & 3.26 \\
\hline 41352 & 3.82 & 1 & 1.87 & 0.72 & 1.02 & 1.8 & 1.47 & 2.63 \\
\hline 41523 & 4.02 & 1 & 1.89 & 0.75 & 1.49 & 1.53 & 1.28 & 3.25 \\
\hline 41532 & 3.89 & 1 & 1.85 & 0.71 & 1.42 & 1.79 & 1.46 & 2.72 \\
\hline 42135 & 2.19 & 1 & 1.57 & 0.82 & 0.72 & 0.69 & 0.59 & 1.34 \\
\hline 42153 & 2.11 & 1 & 1.56 & 0.87 & 0.72 & 1.17 & 1.21 & 1.53 \\
\hline 42315 & 2.05 & 1 & 1.45 & 0.79 & 0.98 & 0.86 & 0.8 & 1.25 \\
\hline 42351 & 1.92 & 1 & 1.48 & 0.9 & 1.14 & 0.93 & 0.79 & 1.03 \\
\hline 42513 & 2.04 & 1 & 1.47 & 0.97 & 0.8 & 0.76 & 0.69 & 1.07 \\
\hline 42531 & 1.97 & 1 & 1.49 & 0.93 & 0.88 & 1.09 & 0.93 & 1.06 \\
\hline 43125 & 3.44 & 1 & 1.53 & 1.99 & 0.97 & 0.86 & 0.81 & 2.41 \\
\hline 43152 & 2.96 & 1 & 1.46 & 1.8 & 0.96 & 1.48 & 1.41 & 1.98 \\
\hline 43215 & 1.86 & 1 & 1.33 & 1.57 & 0.8 & 0.62 & 0.57 & 1.1 \\
\hline 43251 & 1.85 & 1 & 1.31 & 1.49 & 0.91 & 0.82 & 0.7 & 0.86 \\
\hline 43512 & 1.67 & 1 & 1.22 & 1.4 & 0.71 & 0.35 & 0.27 & 0.9 \\
\hline 43521 & 1.7 & 1 & 1.27 & 1.45 & 1.08 & 0.67 & 0.6 & 0.73 \\
\hline 45123 & 1.91 & 1 & 1.43 & 0.78 & 0.36 & 0.33 & 0.25 & 1.28 \\
\hline 45132 & 1.96 & 1 & 1.42 & 0.79 & 0.36 & 0.49 & 0.4 & 1.14 \\
\hline 45213 & 1.96 & 1 & 1.5 & 1.2 & 0.76 & 0.67 & 0.65 & 0.85 \\
\hline 45231 & 1.9 & 1 & 1.44 & 1.2 & 0.69 & 0.93 & 0.78 & 0.86 \\
\hline 45312 & 1.69 & 1 & 1.25 & 0.67 & 0.9 & 0.49 & 0.45 & 0.95 \\
\hline 45321 & 1.65 & 1 & 1.33 & 1.05 & 1.24 & 0.71 & 0.61 & 0.68 \\
\hline
\end{tabular}

Figure 26: vast-2015-mc1-5d results normalized by splatt (serial) (5)

\begin{tabular}{|c|c|c|c|c|c|c|c|c|}
\hline & qsort & splatt & 1-sadilla & 2-sadilla & 3-sadilla & 4-sadilla & quesadilla & radix \\
\hline 51234 & 5.17 & 1 & 2.26 & 0.82 & 0.66 & 0.67 & 0.46 & 3.65 \\
\hline 51243 & 5.08 & 1 & 2.24 & 0.83 & 0.65 & 1.99 & 1.78 & 3.76 \\
\hline 51324 & 5.03 & 1 & 2.24 & 0.81 & 1.19 & 1.05 & 0.8 & 3.48 \\
\hline 51342 & 3.88 & 1 & 1.89 & 0.71 & 1.02 & 1.78 & 1.44 & 2.64 \\
\hline 51423 & 3.94 & 1 & 1.84 & 0.73 & 1.42 & 1.37 & 1.32 & 2.85 \\
\hline 51432 & 3.91 & 1 & 1.83 & 0.72 & 1.5 & 1.78 & 1.56 & 2.73 \\
\hline 52134 & 2.22 & 1 & 1.53 & 0.84 & 0.72 & 0.66 & 0.66 & 1.32 \\
\hline 52143 & 2.1 & 1 & 1.55 & 0.8 & 0.68 & 1.19 & 1.11 & 1.51 \\
\hline 52314 & 2.08 & 1 & 1.47 & 0.84 & 1.01 & 0.83 & 0.81 & 1.25 \\
\hline 52341 & 1.94 & 1 & 1.47 & 0.93 & 1.12 & 0.88 & 0.75 & 1.06 \\
\hline 52413 & 2.05 & 1 & 1.49 & 0.97 & 0.87 & 0.86 & 0.76 & 1.09 \\
\hline 52431 & 1.99 & 1 & 1.45 & 0.92 & 0.82 & 1.04 & 0.9 & 1 \\
\hline 53124 & 3.45 & 1 & 1.51 & 1.99 & 1.05 & 0.94 & 0.71 & 2.36 \\
\hline 53142 & 2.98 & 1 & 1.47 & 1.82 & 0.96 & 1.54 & 1.41 & 2.17 \\
\hline 53214 & 1.89 & 1 & 1.37 & 1.54 & 0.81 & 0.62 & 0.61 & 1.11 \\
\hline 53241 & 1.86 & 1 & 1.3 & 1.52 & 0.88 & 0.77 & 0.7 & 0.93 \\
\hline 53412 & 1.67 & 1 & 1.21 & 1.39 & 0.71 & 0.34 & 0.26 & 0.92 \\
\hline 53421 & 1.71 & 1 & 1.27 & 1.42 & 1.08 & 0.7 & 0.55 & 0.71 \\
\hline 54123 & 2 & 1 & 1.39 & 0.8 & 0.35 & 0.33 & 0.24 & 1.23 \\
\hline 54132 & 1.95 & 1 & 1.4 & 0.76 & 0.35 & 0.48 & 0.4 & 1.17 \\
\hline 54213 & 1.98 & 1 & 1.48 & 1.21 & 0.76 & 0.66 & 0.65 & 0.87 \\
\hline 54231 & 1.89 & 1 & 1.45 & 1.19 & 0.75 & 0.9 & 0.78 & 0.83 \\
\hline 54312 & 1.71 & 1 & 1.25 & 0.66 & 0.92 & 0.53 & 0.44 & 0.94 \\
\hline 54321 & 1.71 & 1 & 1.33 & 1.06 & 1.23 & 0.67 & 0.57 & 0.77 \\
\hline
\end{tabular}


Figure 27: flickr-3d results normalized by splatt (parallel)

$\begin{array}{lcccccc} & \text { qsort } & \text { splatt } & \text { 1-sadilla } & \text { 2-sadilla } & \text { k-sadilla } & \text { radix } \\ 123 & 30.16 & 1 & 0.29 & 0.27 & 0 & 9.83 \\ 132 & 34.61 & 1 & 0.56 & 2.06 & 1.82 & 7.27 \\ 213 & 5.23 & 1 & 1.08 & 0.63 & 0.6 & 0.96 \\ 231 & 5.27 & 1 & 1.05 & 0.96 & 0.91 & 1.02 \\ 312 & 40.67 & 1 & 1.27 & 1.1 & 0.9 & 5.86 \\ 321 & 36.65 & 1 & 1.3 & 4.52 & 4.37 & 5.18\end{array}$

Figure 28: nell-1 results normalized by splatt (parallel)

$\begin{array}{lcccccc} & \text { qsort } & \text { splatt } & \text { 1-sadilla } & \text { 2-sadilla } & \text { k-sadilla } & \text { radix } \\ 123 & 19.94 & 1 & 0.35 & 0.16 & 0 & 7.08 \\ 132 & 25.94 & 1 & 0.46 & 3.69 & 3.5 & 6.4 \\ 213 & 33.9 & 1 & 1.25 & 1.16 & 0.91 & 7.54 \\ 231 & 34.15 & 1 & 1.34 & 5.69 & 5.41 & 6.26 \\ 312 & 8.07 & 1 & 1.07 & 0.75 & 0.76 & 1.16 \\ 321 & 8.18 & 1 & 1.09 & 0.93 & 0.86 & 1.08\end{array}$

Figure 29: nell-2 results normalized by splatt (parallel)

$\begin{array}{lcccccc} & \text { qsort } & \text { splatt } & \text { 1-sadilla } & \text { 2-sadilla } & \text { k-sadilla } & \text { radix } \\ 123 & 28.95 & 1 & 0.39 & 0.24 & 0 & 4.48 \\ 132 & 30.24 & 1 & 0.74 & 2.13 & 1.86 & 2.93 \\ 213 & 38.84 & 1 & 1.26 & 1.13 & 0.8 & 3.88 \\ 231 & 28.38 & 1 & 1.37 & 1.98 & 1.74 & 2.31 \\ 312 & 36.86 & 1 & 1.33 & 1.35 & 1.16 & 2.46 \\ 321 & 35.15 & 1 & 1.41 & 1.69 & 1.54 & 2.09\end{array}$

Figure 30: vast-2015-mc1-3d results normalized by splatt (parallel)

\begin{tabular}{l|c|c|c|ccc} 
& qsort & splatt & 1-sadilla & 2-sadilla & k-sadilla & radix \\
123 & 25.76 & 1 & 0.31 & 0.41 & 0 & 4.63 \\
132 & 25.51 & 1 & 0.38 & 1.68 & 1.49 & 4.22 \\
213 & 35.88 & 1 & 1.31 & 1.46 & 1.1 & 2.59 \\
231 & 33.91 & 1 & 1.39 & 1.92 & 1.66 & 2.3 \\
312 & 3.62 & 1 & 1.29 & 0.14 & 0.11 & 0.59 \\
321 & 2.04 & 1 & 1.47 & 0.11 & $9.8 \cdot 10^{-2}$ & 0.13
\end{tabular}


Figure 31: chicago-crime-comm results normalized by splatt (parallel)

\begin{tabular}{lc|c|c|c|c|c|c} 
& qsort & splatt & 1-sadilla & 2-sadilla & 3-sadilla & k-sadilla & radix \\
1234 & 20.77 & 1 & 0.33 & 0.33 & 0.41 & 0 & 4.34 \\
1243 & 22.91 & 1 & 0.48 & 0.44 & 1.9 & 1.63 & 4.05 \\
1324 & 22.96 & 1 & 0.54 & 1.75 & 1.73 & 1.33 & 3.88 \\
1342 & 23.23 & 1 & 0.53 & 1.71 & 3.02 & 2.46 & 3.72 \\
1423 & 23.05 & 1 & 0.56 & 1.42 & 1.47 & 1.17 & 3.52 \\
1432 & 26.74 & 1 & 0.58 & 1.7 & 2.97 & 2.59 & 3.56 \\
2134 & 22.26 & 1 & 1.24 & 0.86 & 0.94 & 0.6 & 3.17 \\
2143 & 19.48 & 1 & 1.22 & 0.77 & 1.72 & 1.43 & 2.39 \\
2314 & 13.32 & 1 & 1.26 & 0.84 & 0.89 & 0.76 & 1.51 \\
2341 & 13.16 & 1 & 1.3 & 0.95 & 1.11 & 0.97 & 1.29 \\
2413 & 15.66 & 1 & 1.31 & 0.93 & 0.92 & 0.71 & 1.77 \\
2431 & 15.23 & 1 & 1.36 & 1.03 & 1.17 & 0.99 & 1.32 \\
3124 & 28.37 & 1 & 1.14 & 1.03 & 1.1 & 0.68 & 3.2 \\
3142 & 23.35 & 1 & 1.2 & 0.93 & 2.08 & 1.77 & 2.58 \\
3214 & 17.08 & 1 & 1.16 & 1 & 1.03 & 0.78 & 1.67 \\
3241 & 19.92 & 1 & 1.39 & 1.43 & 1.47 & 1.29 & 1.71 \\
3412 & 19.35 & 1 & 1.33 & 1.1 & 1.08 & 0.9 & 1.87 \\
3421 & 19.86 & 1 & 1.34 & 1.36 & 1.36 & 1.17 & 1.53 \\
4123 & 18.49 & 1 & 1.23 & 0.77 & 0.64 & 0.39 & 2.05 \\
4132 & 10.97 & 1 & 1.31 & 0.57 & 0.78 & 0.64 & 1.12 \\
4213 & 9.83 & 1 & 1.38 & 0.64 & 0.55 & 0.43 & 0.98 \\
4231 & 8.49 & 1 & 1.29 & 0.81 & 0.62 & 0.5 & 0.69 \\
4312 & 8.79 & 1 & 1.32 & 0.52 & 0.46 & 0.37 & 0.8 \\
4321 & 9.14 & 1 & 1.37 & 0.81 & 0.62 & 0.52 & 0.69 \\
& & & & & & &
\end{tabular}

Figure 32: delicious-4d results normalized by splatt (parallel)

\begin{tabular}{l|c|c|c|c|c|c|c} 
& qsort & splatt & 1-sadilla & 2-sadilla & 3-sadilla & k-sadilla & radix \\
\hline 1234 & 22 & 1 & 0.27 & 0.24 & 0.29 & 0 & 8.43 \\
1243 & 22.77 & 1 & 0.29 & 0.24 & 2.22 & 1.98 & 8.09 \\
1324 & 27.1 & 1 & 0.45 & 2.09 & 2.1 & 1.87 & 6.87 \\
1342 & 28.57 & 1 & 0.48 & 2.17 & 3.67 & 3.45 & 6.94 \\
1423 & 25.41 & 1 & 0.43 & 1.79 & 1.81 & 1.59 & 7.22 \\
1432 & 26.43 & 1 & 0.46 & 1.88 & 3.77 & 3.52 & 7.04 \\
2134 & 7.87 & 1 & 1.02 & 0.67 & 0.73 & 0.64 & 1.61 \\
2143 & 7.85 & 1 & 1.04 & 0.69 & 1.05 & 1.08 & 1.54 \\
2314 & 8.32 & 1 & 1.1 & 1.25 & 1.2 & 1.16 & 1.63 \\
2341 & 8.17 & 1 & 1.01 & 1.21 & 1.33 & 1.29 & 1.51 \\
2413 & 8.07 & 1 & 1.04 & 0.87 & 0.85 & 0.81 & 1.46 \\
2431 & 7.82 & 1 & 1.04 & 0.89 & 1.28 & 1.23 & 1.42 \\
3124 & 27.34 & 1 & 1.19 & 1.01 & 0.96 & 0.79 & 4.37 \\
3142 & 29.79 & 1 & 1.22 & 1.25 & 2.6 & 2.41 & 4.5 \\
3214 & 29.22 & 1 & 1.31 & 2.89 & 2.82 & 2.64 & 4.1 \\
3241 & 29.22 & 1 & 1.29 & 3.02 & 3.4 & 3.19 & 3.8 \\
3412 & 28.52 & 1 & 1.34 & 2.65 & 1.69 & 1.46 & 4.32 \\
3421 & 27.02 & 1 & 1.24 & 3.7 & 3.24 & 2.98 & 3.67 \\
4123 & 45.13 & 1 & 1.39 & 1.23 & 1.23 & 0.95 & 9.09 \\
4132 & 42.01 & 1 & 1.3 & 1.14 & 3.94 & 3.62 & 7.74 \\
4213 & 35.6 & 1 & 1.31 & 3.59 & 3.61 & 3.36 & 6.29 \\
4231 & 36.14 & 1 & 1.42 & 3.67 & 5.61 & 5.27 & 6.03 \\
4312 & 36.13 & 1 & 1.31 & 2 & 2.21 & 1.89 & 5.58 \\
4321 & 38.52 & 1 & 1.35 & 2.17 & 4.62 & 4.48 & 5.2 \\
& & & & & & & \\
\end{tabular}


Figure 33: enron results normalized by splatt (parallel)

\begin{tabular}{ll|c|c|c|c|c|c} 
& qsort & splatt & 1-sadilla & 2-sadilla & 3-sadilla & k-sadilla & radix \\
1234 & 16.97 & 1 & 0.74 & 0.63 & 0.28 & 0 & 3.35 \\
1243 & 11.96 & 1 & 1.1 & 1 & 0.81 & 0.62 & 1.62 \\
1324 & 12.33 & 1 & 1.03 & 1.31 & 1.22 & 1.04 & 1.84 \\
1342 & 11.91 & 1 & 1.06 & 1.42 & 2.02 & 1.92 & 1.64 \\
1423 & 11.06 & 1 & 1.15 & 0.88 & 0.8 & 0.62 & 1.37 \\
1432 & 10.94 & 1 & 1.13 & 1.28 & 1.46 & 1.39 & 1.33 \\
2134 & 34.84 & 1 & 1.26 & 1.38 & 1.28 & 0.9 & 4.46 \\
2143 & 35.46 & 1 & 1.4 & 1.64 & 2.39 & 2.08 & 3.5 \\
2314 & 28.28 & 1 & 1.32 & 2.39 & 2.38 & 2.08 & 3.81 \\
2341 & 29.33 & 1 & 1.3 & 2.58 & 3.55 & 3.14 & 4.01 \\
2413 & 31.84 & 1 & 1.35 & 1.73 & 1.67 & 1.41 & 3.19 \\
2431 & 33.92 & 1 & 1.35 & 1.97 & 3.22 & 2.91 & 3.68 \\
3124 & 29.62 & 1 & 1.24 & 1.23 & 1.23 & 1 & 3.25 \\
3142 & 27.79 & 1 & 1.28 & 1.36 & 2.34 & 2.16 & 2.84 \\
3214 & 26.36 & 1 & 1.26 & 1.97 & 1.87 & 1.58 & 2.86 \\
3241 & 27.02 & 1 & 1.28 & 2.13 & 2.57 & 2.32 & 3.01 \\
3412 & 24.39 & 1 & 1.24 & 1.72 & 1.57 & 1.42 & 2.47 \\
3421 & 26.19 & 1 & 1.27 & 1.98 & 2.47 & 2.15 & 2.85 \\
4123 & 45.37 & 1 & 1.34 & 1.26 & 1.12 & 0.9 & 4.47 \\
4132 & 35.29 & 1 & 1.39 & 1.24 & 2.48 & 2.25 & 3.45 \\
4213 & 38.31 & 1 & 1.39 & 1.83 & 1.81 & 1.59 & 3.56 \\
4231 & 38.51 & 1 & 1.35 & 1.96 & 3.27 & 3.02 & 3.74 \\
4312 & 32.38 & 1 & 1.31 & 1.8 & 1.79 & 1.64 & 2.9 \\
4321 & 34.32 & 1 & 1.31 & 2.01 & 2.87 & 2.57 & 3.2 \\
& & & & & & &
\end{tabular}

Figure 34: flickr-4d results normalized by splatt (parallel)

\begin{tabular}{|c|c|c|c|c|c|c|c|}
\hline & qsort & splatt & 1-sadilla & 2-sadilla & 3-sadilla & k-sadilla & radix \\
\hline 1234 & 22.94 & 1 & 0.27 & 0.24 & 0.3 & 0 & 9.65 \\
\hline 1243 & 23.13 & 1 & 0.28 & 0.25 & 2.09 & 1.82 & 8.61 \\
\hline 1324 & 26.8 & 1 & 0.47 & 1.93 & 1.97 & 1.7 & 7 \\
\hline 1342 & 27.1 & 1 & 0.52 & 1.9 & 3.38 & 3.17 & 7.13 \\
\hline 1423 & 23.8 & 1 & 0.31 & 2.01 & 2.02 & 1.79 & 9.63 \\
\hline 1432 & 24.55 & 1 & 0.47 & 1.87 & 3.57 & 3.38 & 7.58 \\
\hline 2134 & 4.42 & 1 & 1.03 & 0.59 & 0.57 & 0.56 & 1.03 \\
\hline 2143 & 4.51 & 1 & 1.03 & 0.58 & 0.77 & 0.75 & 1.02 \\
\hline 2314 & 4.48 & 1 & 1.03 & 0.88 & 0.91 & 0.88 & 1.13 \\
\hline 2341 & 4.4 & 1 & 1.02 & 0.91 & 1.04 & 1.02 & 1.14 \\
\hline 2413 & 4.44 & 1 & 1.06 & 0.68 & 0.67 & 0.64 & 1.01 \\
\hline 2431 & 4.28 & 1 & 1.03 & 0.68 & 0.87 & 0.85 & 0.97 \\
\hline 3124 & 33.11 & 1 & 1.22 & 1.12 & 1.13 & 0.9 & 5.69 \\
\hline 3142 & 31.63 & 1 & 1.17 & 1.09 & 2.5 & 2.29 & 5.69 \\
\hline 3214 & 29.92 & 1 & 1.27 & 4.04 & 4.22 & 4.04 & 5.43 \\
\hline 3241 & 29.43 & 1 & 1.26 & 4.1 & 4.63 & 4.48 & 5.08 \\
\hline 3412 & 28.29 & 1 & 1.33 & 2.16 & 1.63 & 1.45 & 5.11 \\
\hline 3421 & 29 & 1 & 1.4 & 2.65 & 4.6 & 4.4 & 5.26 \\
\hline 4123 & 36.73 & 1 & 1.23 & 1.12 & 1.1 & 0.86 & 9.06 \\
\hline 4132 & 37.87 & 1 & 1.33 & 1.16 & 3.03 & 3 & 7.72 \\
\hline 4213 & 31.15 & 1 & 1.32 & 4.84 & 4.7 & 4.5 & 6.98 \\
\hline 4231 & 31.86 & 1 & 1.32 & 4.81 & 7.08 & 6.82 & 7.58 \\
\hline 4312 & 32.04 & 1 & 1.32 & 1.83 & 1.82 & 1.64 & 5.99 \\
\hline 4321 & 31.65 & 1 & 1.28 & 1.82 & 5.27 & 5.09 & 5.76 \\
\hline
\end{tabular}


Figure 35: nips results normalized by splatt (parallel)

\begin{tabular}{l|c|c|c|c|c|c|c} 
& qsort & splatt & 1-sadilla & 2-sadilla & 3-sadilla & k-sadilla & radix \\
1234 & 52.96 & 1 & 0.78 & 0.67 & 0.43 & 0 & 5.75 \\
1243 & 50.01 & 1 & 0.84 & 0.76 & 3.07 & 2.43 & 5.78 \\
1324 & 16.71 & 1 & 1.12 & 1.18 & 1.13 & 1.06 & 1.63 \\
1342 & 16.79 & 1 & 1.13 & 1.17 & 1.93 & 1.91 & 1.71 \\
1423 & 50.82 & 1 & 0.88 & 3.45 & 3.23 & 2.45 & 5.76 \\
1432 & 12.27 & 1 & 1.64 & 1.44 & 1.44 & 1.3 & 1.1 \\
2134 & 68.12 & 1 & 1.41 & 1.21 & 1.07 & 0.91 & 4.4 \\
2143 & 66.32 & 1 & 1.59 & 1.39 & 3.34 & 2.67 & 4.62 \\
2314 & 50.48 & 1 & 1.47 & 3.15 & 3.09 & 2.85 & 3.35 \\
2341 & 50.55 & 1 & 1.33 & 3.17 & 3.32 & 3 & 3.61 \\
2413 & 66.56 & 1 & 1.59 & 2.12 & 2.03 & 1.07 & 4.34 \\
2431 & 56.64 & 1 & 1.59 & 1.9 & 4.11 & 3.22 & 3.81 \\
3124 & 40.3 & 1 & 1.43 & 1.29 & 1.39 & 1.19 & 2.08 \\
3142 & 39.82 & 1 & 1.36 & 1.36 & 2.65 & 2.27 & 2.03 \\
3214 & 40.35 & 1 & 1.6 & 1.44 & 1.41 & 1.1 & 1.76 \\
3241 & 39.29 & 1 & 1.39 & 1.49 & 1.59 & 1.42 & 1.71 \\
3412 & 41.58 & 1 & 1.59 & 1.57 & 1.55 & 1.13 & 2.01 \\
3421 & 38.93 & 1 & 1.42 & 1.53 & 1.74 & 1.47 & 1.89 \\
4123 & 25.39 & 1 & 1.84 & 0.71 & 0.66 & 0.57 & 2.74 \\
4132 & 14.84 & 1 & 1.92 & 1.44 & 1.12 & 1.04 & 1.39 \\
4213 & 16.52 & 1 & 1.39 & 0.56 & 0.58 & 0.4 & 1.28 \\
4231 & 17.58 & 1 & 1.56 & 0.67 & 1.39 & 1.3 & 1.37 \\
4312 & 16.33 & 1 & 1.66 & 0.78 & 0.8 & 0.67 & 0.95 \\
4321 & 15.33 & 1 & 1.35 & 0.78 & 0.84 & 0.66 & 0.77 \\
& & & & & & &
\end{tabular}

Figure 36: uber results normalized by splatt (parallel)

\begin{tabular}{|c|c|c|c|c|c|c|c|}
\hline & qsort & splatt & 1-sadilla & 2-sadilla & 3-sadilla & k-sadilla & radix \\
\hline 1234 & 54.22 & 1 & 0.93 & 0.66 & 0.55 & 0 & 3.82 \\
\hline 1243 & 44.93 & 1 & 1.1 & 0.79 & 1.77 & 1.61 & 2.06 \\
\hline 1324 & 39.21 & 1 & 1.12 & 2.09 & 1.96 & 1.61 & 2.16 \\
\hline 1342 & 41.63 & 1 & 1.09 & 2.09 & 3.22 & 3.01 & 2 \\
\hline 1423 & 40.58 & 1 & 1.13 & 1.56 & 1.57 & 1.21 & 1.67 \\
\hline 1432 & 45.75 & 1 & 1.13 & 2.09 & 2.77 & 2.63 & 1.73 \\
\hline 2134 & 37.23 & 1 & 1.55 & 0.71 & 0.63 & 0.29 & 1.8 \\
\hline 2143 & 30.16 & 1 & 2.02 & 0.69 & 1.17 & 1.18 & 1.15 \\
\hline 2314 & 18.64 & 1 & 1.51 & 0.7 & 0.56 & 0.52 & 0.91 \\
\hline 2341 & 18.12 & 1 & 1.51 & 1.04 & 0.81 & 0.69 & 0.72 \\
\hline 2413 & 18.28 & 1 & 1.47 & 0.56 & 0.42 & 0.38 & 0.68 \\
\hline 2431 & 19.51 & 1 & 1.42 & 0.94 & 0.78 & 0.59 & 0.61 \\
\hline 3124 & 59.82 & 1 & 1.71 & 2.09 & 1.56 & 0.77 & 2.55 \\
\hline 3142 & 46.19 & 1 & 1.68 & 2.2 & 2.04 & 2.07 & 1.74 \\
\hline 3214 & 40.56 & 1 & 1.63 & 1.47 & 0.94 & 0.67 & 1.77 \\
\hline 3241 & 38.01 & 1 & 1.57 & 2.44 & 1.61 & 1.35 & 1.35 \\
\hline 3412 & 37.55 & 1 & 1.64 & 1.04 & 0.86 & 0.83 & 1.26 \\
\hline 3421 & 38.2 & 1 & 1.66 & 1.37 & 1.26 & 0.97 & 1.17 \\
\hline 4123 & 86.7 & 1 & 1.95 & 3.06 & 1.91 & 0.83 & 3.08 \\
\hline 4132 & 63.18 & 1 & 1.94 & 4.09 & 2.56 & 2.4 & 2 \\
\hline 4213 & 54.58 & 1 & 1.73 & 2.54 & 1.23 & 0.77 & 2 \\
\hline 4231 & 52.89 & 1 & 1.82 & 4.87 & 1.98 & 1.52 & 1.58 \\
\hline 4312 & 49.2 & 1 & 1.65 & 1.51 & 1.04 & 0.93 & 1.32 \\
\hline 4321 & 50.71 & 1 & 1.76 & 2.58 & 1.57 & 1.09 & 1.3 \\
\hline
\end{tabular}


Figure 37: lbnl-network results normalized by splatt (parallel) (1)

$\begin{array}{lcccccccc} & \text { qsort } & \text { splatt } & \text { 1-sadilla } & \text { 2-sadilla } & \text { 3-sadilla } & \text { 4-sadilla } & \text { k-sadilla } & \text { radix } \\ 12345 & 5.6 & 1 & 0.9 & 0.71 & 0.61 & 0.37 & 0 & 2.57 \\ 12354 & 4.12 & 1 & 0.84 & 0.7 & 0.48 & 1.2 & 1.06 & 1.88 \\ 12435 & 3.97 & 1 & 0.92 & 0.73 & 0.67 & 0.66 & 0.34 & 1.55 \\ 12453 & 3.46 & 1 & 0.74 & 0.6 & 0.54 & 1.39 & 1.36 & 1.46 \\ 12534 & 3.66 & 1 & 0.75 & 0.51 & 1.07 & 1.13 & 0.98 & 1.5 \\ 12543 & 4.19 & 1 & 0.9 & 0.65 & 1.16 & 1.66 & 1.62 & 1.87 \\ 13245 & 4.51 & 1 & 0.97 & 0.99 & 0.92 & 0.72 & 0.44 & 1.76 \\ 13254 & 4.33 & 1 & 1.09 & 1.26 & 0.92 & 1.44 & 1.5 & 1.86 \\ 13425 & 4.56 & 1 & 0.96 & 1.21 & 1.24 & 1.09 & 0.8 & 1.93 \\ 13452 & 4.13 & 1 & 0.73 & 1.08 & 1.1 & 2.03 & 2.13 & 1.78 \\ 13524 & 3.88 & 1 & 0.87 & 0.86 & 1.62 & 1.58 & 1.62 & 1.66 \\ 13542 & 4.6 & 1 & 0.83 & 0.99 & 1.68 & 2.25 & 2.24 & 1.96 \\ 14235 & 3.97 & 1 & 0.78 & 0.71 & 0.67 & 0.64 & 0.35 & 1.58 \\ 14253 & 4.34 & 1 & 0.81 & 0.75 & 0.68 & 1.51 & 1.39 & 1.82 \\ 14325 & 4.15 & 1 & 0.77 & 0.77 & 0.97 & 1.01 & 0.7 & 1.52 \\ 14352 & 4.54 & 1 & 0.82 & 0.9 & 1.1 & 2.1 & 2.06 & 1.74 \\ 14523 & 5.55 & 1 & 0.88 & 0.89 & 1.98 & 2.02 & 2.16 & 2.18 \\ 14532 & 5.74 & 1 & 0.93 & 0.91 & 2.31 & 2.71 & 2.73 & 2.23 \\ 15234 & 5.7 & 1 & 0.72 & 1.49 & 1.61 & 1.63 & 1.42 & 2.25 \\ 15243 & 5.97 & 1 & 0.97 & 1.55 & 1.59 & 1.85 & 2.02 & 2.35 \\ 15324 & 5.62 & 1 & 0.79 & 1.64 & 2.16 & 2.12 & 2.05 & 2.3 \\ 15342 & 6.28 & 1 & 1.01 & 1.9 & 2.52 & 2.95 & 2.83 & 2.43 \\ 15423 & 6.28 & 1 & 0.9 & 1.85 & 2.13 & 2.39 & 2.16 & 2.57 \\ 15432 & 5.65 & 1 & 0.74 & 1.57 & 2.02 & 2.64 & 2.48 & 2.19\end{array}$

Figure 38: lbnl-network results normalized by splatt (parallel) (2)

$\begin{array}{lccccccccc} & \text { qsort } & \text { splatt } & \text { 1-sadilla } & \text { 2-sadilla } & \text { 3-sadilla } & \text { 4-sadilla } & \text { k-sadilla } & \text { radix } \\ 21345 & 4.86 & 1 & 1.14 & 0.85 & 0.48 & 0.37 & 5 \cdot 10^{-2} & 1.74 \\ 21354 & 3.73 & 1 & 1.19 & 0.81 & 0.43 & 0.82 & 0.79 & 1.24 \\ 21435 & 3.81 & 1 & 1.02 & 0.83 & 0.51 & 0.5 & 0.3 & 1.32 \\ 21453 & 3.4 & 1 & 0.89 & 0.75 & 0.45 & 1.01 & 1 & 1.02 \\ 21534 & 3.89 & 1 & 1.1 & 0.76 & 0.91 & 0.83 & 0.79 & 1.26 \\ 21543 & 3.76 & 1 & 1.22 & 0.88 & 0.88 & 1.16 & 1.09 & 1.25 \\ 23145 & 3.29 & 1 & 1.11 & 0.4 & 0.31 & 0.24 & 7.2 \cdot 10^{-2} & 1.1 \\ 23154 & 3.11 & 1 & 1.21 & 0.58 & 0.35 & 0.71 & 0.64 & 1.11 \\ 23415 & 2.98 & 1 & 1.01 & 0.47 & 0.31 & 0.26 & 0.14 & 0.94 \\ 23451 & 3.09 & 1 & 1.16 & 0.51 & 0.28 & 0.98 & 0.96 & 0.99 \\ 23514 & 3.23 & 1 & 1.01 & 0.48 & 1.06 & 1.16 & 1.01 & 1.11 \\ 23541 & 3.15 & 1 & 1.03 & 0.47 & 1.12 & 1.02 & 1.02 & 1.06 \\ 24135 & 3.2 & 1 & 1.1 & 0.21 & 0.19 & 0.17 & 7.4 \cdot 10^{-2} & 1.08 \\ 24153 & 3.35 & 1 & 1.17 & 0.2 & 0.18 & 0.71 & 0.64 & 1.09 \\ 24315 & 3.26 & 1 & 1.07 & 0.26 & 0.31 & 0.22 & 0.14 & 1.08 \\ 24351 & 3.3 & 1 & 1.12 & 0.24 & 0.24 & 1.05 & 1.09 & 1.05 \\ 24513 & 3.65 & 1 & 1.23 & 0.23 & 1.18 & 1.12 & 1.06 & 1.19 \\ 24531 & 3.56 & 1 & 1.17 & 0.22 & 1.07 & 1.1 & 1.08 & 1.16 \\ 25134 & 3.18 & 1 & 0.9 & 0.9 & 0.96 & 1.01 & 0.92 & 1.02 \\ 25143 & 3.67 & 1 & 1.15 & 1.03 & 1.1 & 1.17 & 1.2 & 1.16 \\ 25314 & 4.01 & 1 & 1.29 & 1.17 & 1.33 & 1.3 & 1.25 & 1.37 \\ 25341 & 3.2 & 1 & 0.79 & 1 & 1.07 & 1.03 & 0.96 & 1.13 \\ 25413 & 3.89 & 1 & 1.22 & 1.18 & 1.32 & 1.21 & 1.2 & 1.32 \\ 25431 & 4.02 & 1 & 1.09 & 1.09 & 1.24 & 1.15 & 1.27 & 1.3\end{array}$


Figure 39: lbnl-network results normalized by splatt (parallel) (3)

\begin{tabular}{|c|c|c|c|c|c|c|c|c|}
\hline & qsort & splatt & 1-sadilla & 2-sadilla & 3-sadilla & 4-sadilla & k-sadilla & radix \\
\hline 31245 & 9.72 & 1 & 0.95 & 0.83 & 0.77 & 0.61 & 0.1 & 3.21 \\
\hline 31254 & 6.56 & 1 & 0.91 & 0.9 & 0.57 & 1.34 & 1.45 & 2.05 \\
\hline 31425 & 6.26 & 1 & 1.03 & 0.84 & 0.82 & 0.68 & 0.44 & 2.06 \\
\hline 31452 & 7.26 & 1 & 1.02 & 0.88 & 1.02 & 1.91 & 2.02 & 2.45 \\
\hline 31524 & 6.41 & 1 & 0.85 & 0.61 & 1.38 & 1.27 & 1.25 & 2.21 \\
\hline 31542 & 6.53 & 1 & 0.96 & 0.62 & 1.47 & 1.64 & 1.72 & 2.27 \\
\hline 32145 & 5.89 & 1 & 0.86 & 0.58 & 0.5 & 0.43 & 0.13 & 1.85 \\
\hline 32154 & 6.08 & 1 & 1.03 & 0.79 & 0.59 & 1.24 & 1.3 & 1.98 \\
\hline 32415 & 5.77 & 1 & 1.02 & 0.82 & 0.67 & 0.49 & 0.26 & 1.98 \\
\hline 32451 & 6.62 & 1 & 1.05 & 0.75 & 0.6 & 1.95 & 1.99 & 2.2 \\
\hline 32514 & 5.97 & 1 & 0.84 & 0.64 & 2.16 & 2.05 & 1.8 & 1.93 \\
\hline 32541 & 6.06 & 1 & 0.97 & 0.67 & 1.79 & 2 & 1.81 & 2.04 \\
\hline 34125 & 6.24 & 1 & 1.12 & 0.63 & 0.56 & 0.41 & 0.14 & 1.88 \\
\hline 34152 & 6.56 & 1 & 1.05 & 0.77 & 0.64 & 1.28 & 1.26 & 2.09 \\
\hline 34215 & 4.76 & 1 & 0.82 & 0.78 & 0.48 & 0.41 & 0.23 & 1.59 \\
\hline 34251 & 5.22 & 1 & 0.99 & 0.84 & 0.46 & 1.74 & 1.58 & 1.76 \\
\hline 34512 & 6.45 & 1 & 1.05 & 0.84 & 2.14 & 2.16 & 1.88 & 2.1 \\
\hline 34521 & 6.01 & 1 & 0.83 & 0.76 & 2.09 & 1.79 & 1.85 & 2.02 \\
\hline 35124 & 8.43 & 1 & 1.05 & 2.39 & 2.6 & 2.36 & 2.6 & 2.68 \\
\hline 35142 & 9.34 & 1 & 1.2 & 3.02 & 2.78 & 3.43 & 2.97 & 2.99 \\
\hline 35214 & 8.04 & 1 & 1.04 & 2.37 & 2.48 & 2.48 & 2.47 & 2.93 \\
\hline 35241 & 8.5 & 1 & 1.08 & 2.68 & 2.75 & 2.55 & 2.68 & 2.62 \\
\hline 35412 & 8.66 & 1 & 1 & 2.44 & 2.77 & 2.75 & 2.67 & 2.86 \\
\hline 35421 & 9.11 & 1 & 1.04 & 2.61 & 3 & 2.78 & 2.86 & 3.03 \\
\hline
\end{tabular}

Figure 40: lbnl-network results normalized by splatt (parallel) (4)

\begin{tabular}{l|ccccccccc} 
& qsort & splatt & 1-sadilla & 2-sadilla & 3-sadilla & 4-sadilla & k-sadilla & radix \\
41235 & 6.61 & 1 & 1.24 & 0.62 & 0.4 & 0.34 & $7.7 \cdot 10^{-2}$ & 2.1 \\
41253 & 6.2 & 1 & 1.04 & 0.53 & 0.33 & 1.25 & 1.24 & 1.98 \\
41325 & 4.17 & 1 & 0.99 & 0.57 & 0.46 & 0.4 & 0.26 & 1.27 \\
41352 & 4.57 & 1 & 0.99 & 0.62 & 0.56 & 1.14 & 1.18 & 1.5 \\
41523 & 5.02 & 1 & 1.03 & 0.54 & 1.07 & 1.06 & 1.02 & 1.71 \\
41532 & 5.12 & 1 & 1 & 0.55 & 1.09 & 1.24 & 1.28 & 1.67 \\
42135 & 3.67 & 1 & 1.13 & 0.21 & 0.2 & 0.18 & $8 \cdot 10^{-2}$ & 1.09 \\
42153 & 3.88 & 1 & 0.97 & 0.21 & 0.17 & 0.73 & 0.73 & 1.21 \\
42315 & 3.06 & 1 & 0.84 & 0.21 & 0.26 & 0.2 & 0.18 & 0.98 \\
42351 & 3.99 & 1 & 1 & 0.27 & 0.27 & 1.23 & 1.21 & 1.28 \\
42513 & 3.68 & 1 & 0.89 & 0.19 & 1.2 & 1.27 & 1.14 & 1.17 \\
42531 & 3.74 & 1 & 0.82 & 0.2 & 1.2 & 1.17 & 1.22 & 1.15 \\
43125 & 3.28 & 1 & 1.07 & 0.56 & 0.31 & 0.24 & $7.1 \cdot 10^{-2}$ & 1.01 \\
43152 & 2.87 & 1 & 0.86 & 0.5 & 0.3 & 0.61 & 0.55 & 0.92 \\
43215 & 3.17 & 1 & 0.98 & 0.75 & 0.3 & 0.28 & 0.14 & 1.03 \\
43251 & 3.88 & 1 & 1.04 & 0.81 & 0.34 & 1.21 & 1.23 & 1.15 \\
43512 & 3.64 & 1 & 1.24 & 0.71 & 1.07 & 1.24 & 1.11 & 1.18 \\
43521 & 3.63 & 1 & 1.24 & 0.63 & 1.17 & 1.07 & 1.04 & 1.18 \\
45123 & 4.47 & 1 & 0.88 & 1.23 & 1.23 & 1.22 & 1.19 & 1.34 \\
45132 & 4.28 & 1 & 1.13 & 1.22 & 1.22 & 1.51 & 1.48 & 1.36 \\
45213 & 4.37 & 1 & 1.18 & 1.27 & 1.38 & 1.41 & 1.3 & 1.49 \\
45231 & 4.72 & 1 & 1.1 & 1.3 & 1.37 & 1.53 & 1.39 & 1.57 \\
45312 & 4.4 & 1 & 0.98 & 1.31 & 1.32 & 1.37 & 1.25 & 1.32 \\
45321 & 4.27 & 1 & 0.89 & 1.29 & 1.39 & 1.28 & 1.39 & 1.36
\end{tabular}


Figure 41: lbnl-network results normalized by splatt (parallel) (5)

\begin{tabular}{|c|c|c|c|c|c|c|c|c|}
\hline & qsort & splatt & 1-sadilla & 2-sadilla & 3-sadilla & 4-sadilla & k-sadilla & radix \\
\hline 51234 & 1.88 & 1 & 0.96 & 0.53 & 0.5 & 0.5 & 0.57 & 0.61 \\
\hline 51243 & 1.86 & 1 & 1.02 & 0.51 & 0.5 & 0.6 & 0.61 & 0.58 \\
\hline 51324 & 1.86 & 1 & 1 & 0.5 & 0.61 & 0.61 & 0.58 & 0.57 \\
\hline 51342 & 1.86 & 1 & 1.03 & 0.49 & 0.62 & 0.68 & 0.66 & 0.6 \\
\hline 51423 & 1.89 & 1 & 1.01 & 0.5 & 0.59 & 0.6 & 0.58 & 0.59 \\
\hline 51432 & 1.93 & 1 & 1.04 & 0.56 & 0.61 & 0.69 & 0.69 & 0.59 \\
\hline 52134 & 1.95 & 1 & 1.04 & 0.57 & 0.55 & 0.56 & 0.53 & 0.61 \\
\hline 52143 & 1.88 & 1 & 1 & 0.55 & 0.55 & 0.6 & 0.57 & 0.57 \\
\hline 52314 & 1.91 & 1 & 1.05 & 0.54 & 0.57 & 0.57 & 0.56 & 0.64 \\
\hline 52341 & 1.85 & 1 & 1.02 & 0.54 & 0.55 & 0.59 & 0.56 & 0.6 \\
\hline 52413 & 1.87 & 1 & 0.98 & 0.54 & 0.55 & 0.55 & 0.57 & 0.58 \\
\hline 52431 & 1.88 & 1 & 0.98 & 0.55 & 0.66 & 0.58 & 0.59 & 0.58 \\
\hline 53124 & 1.92 & 1 & 1.06 & 0.55 & 0.54 & 0.53 & 0.53 & 0.62 \\
\hline 53142 & 1.92 & 1 & 1.04 & 0.55 & 0.55 & 0.66 & 0.61 & 0.63 \\
\hline 53214 & 1.87 & 1 & 0.99 & 0.56 & 0.56 & 0.58 & 0.59 & 0.62 \\
\hline 53241 & 1.88 & 1 & 1.01 & 0.54 & 0.57 & 0.58 & 0.56 & 0.56 \\
\hline 53412 & 1.93 & 1 & 1.04 & 0.55 & 0.56 & 0.56 & 0.6 & 0.61 \\
\hline 53421 & 1.79 & 1 & 0.99 & 0.51 & 0.55 & 0.54 & 0.55 & 0.58 \\
\hline 54123 & 1.94 & 1 & 1.01 & 0.57 & 0.55 & 0.53 & 0.57 & 0.62 \\
\hline 54132 & 1.91 & 1 & 1.06 & 0.52 & 0.54 & 0.62 & 0.6 & 0.64 \\
\hline 54213 & 1.87 & 1 & 0.98 & 0.53 & 0.63 & 0.56 & 0.55 & 0.58 \\
\hline 54231 & 1.87 & 1 & 0.99 & 0.55 & 0.61 & 0.61 & 0.59 & 0.58 \\
\hline 54312 & 1.92 & 1 & 1.04 & 0.52 & 0.55 & 0.57 & 0.59 & 0.59 \\
\hline 54321 & 1.86 & 1 & 1.03 & 0.53 & 0.58 & 0.6 & 0.57 & 0.56 \\
\hline
\end{tabular}

Figure 42: vast-2015-mc1-5d results normalized by splatt (parallel) (1)

\begin{tabular}{|c|c|c|c|c|c|c|c|c|}
\hline & qsort & splatt & 1-sadilla & 2-sadilla & 3-sadilla & 4-sadilla & k-sadilla & radix \\
\hline 12345 & 19.99 & 1 & 0.29 & 0.27 & 0.27 & 0.28 & 0 & 6.29 \\
\hline 12354 & 19.86 & 1 & 0.28 & 0.28 & 0.27 & 2.17 & 1.9 & 6.24 \\
\hline 12435 & 19.58 & 1 & 0.28 & 0.27 & 2.14 & 2.17 & 1.9 & 6.14 \\
\hline 12453 & 19.43 & 1 & 0.28 & 0.26 & 2.17 & 4.14 & 3.75 & 6.3 \\
\hline 12534 & 19.5 & 1 & 0.29 & 0.27 & 2.16 & 2.2 & 1.86 & 6.25 \\
\hline 12543 & 19.49 & 1 & 0.28 & 0.26 & 2.14 & 4.04 & 3.76 & 6.07 \\
\hline 13245 & 19.23 & 1 & 0.33 & 1.41 & 1.52 & 1.52 & 1.22 & 5.92 \\
\hline 13254 & 19.13 & 1 & 0.33 & 1.47 & 1.54 & 3.4 & 3.06 & 5.76 \\
\hline 13425 & 21.28 & 1 & 0.44 & 1.31 & 2.36 & 2.43 & 2.18 & 4.41 \\
\hline 13452 & 21.23 & 1 & 0.45 & 1.27 & 2.32 & 3.61 & 3.34 & 4.29 \\
\hline 13524 & 22.13 & 1 & 0.44 & 1.3 & 2.42 & 2.43 & 2.21 & 4.38 \\
\hline 13542 & 21.97 & 1 & 0.45 & 1.28 & 2.29 & 3.57 & 3.33 & 4.4 \\
\hline 14235 & 21.99 & 1 & 0.41 & 1.42 & 1.47 & 1.51 & 1.23 & 4.65 \\
\hline 14253 & 23.11 & 1 & 0.41 & 1.46 & 1.48 & 3.06 & 2.8 & 4.73 \\
\hline 14325 & 22.74 & 1 & 0.42 & 1.44 & 2.49 & 2.57 & 2.3 & 4.68 \\
\hline 14352 & 22.65 & 1 & 0.42 & 1.41 & 2.43 & 3.75 & 3.57 & 4.54 \\
\hline 14523 & 22.3 & 1 & 0.42 & 1.4 & 2.71 & 2.65 & 2.48 & 4.62 \\
\hline 14532 & 22.42 & 1 & 0.42 & 1.42 & 2.72 & 3.69 & 3.58 & 4.6 \\
\hline 15234 & 23.33 & 1 & 0.41 & 1.45 & 1.49 & 1.51 & 1.24 & 4.74 \\
\hline 15243 & 23.33 & 1 & 0.41 & 1.44 & 1.49 & 3.1 & 2.79 & 4.9 \\
\hline 15324 & 22.05 & 1 & 0.42 & 1.43 & 2.47 & 2.49 & 2.33 & 4.68 \\
\hline 15342 & 22.87 & 1 & 0.43 & 1.48 & 2.51 & 3.83 & 3.55 & 4.61 \\
\hline 15423 & 23.21 & 1 & 0.43 & 1.48 & 2.84 & 2.84 & 2.48 & 4.68 \\
\hline 15432 & 22.6 & 1 & 0.42 & 1.41 & 2.67 & 3.77 & 3.5 & 4.58 \\
\hline
\end{tabular}


Figure 43: vast-2015-mc1-5d results normalized by splatt (parallel) (2)

\begin{tabular}{lcccccccc} 
& qsort & splatt & 1-sadilla & 2-sadilla & 3-sadilla & 4-sadilla & k-sadilla & radix \\
21345 & 27.03 & 1 & 1.17 & 1.22 & 1.2 & 1.24 & 1.01 & 4.27 \\
21354 & 27.35 & 1 & 1.17 & 1.24 & 1.21 & 2.89 & 2.66 & 4.2 \\
21435 & 27.9 & 1 & 1.2 & 1.23 & 2.89 & 2.94 & 2.85 & 4.17 \\
21453 & 26.41 & 1 & 1.16 & 1.22 & 2.9 & 4.49 & 4.3 & 4.08 \\
21534 & 26.3 & 1 & 1.16 & 1.26 & 2.95 & 2.92 & 2.67 & 4.1 \\
21543 & 28.65 & 1 & 1.21 & 1.3 & 2.96 & 4.71 & 4.53 & 4.32 \\
23145 & 25.02 & 1 & 1.16 & 1.64 & 1.72 & 1.67 & 1.61 & 3.7 \\
23154 & 24.95 & 1 & 1.16 & 1.66 & 1.67 & 3.11 & 2.97 & 3.73 \\
23415 & 23.57 & 1 & 1.15 & 1.69 & 1.94 & 1.97 & 1.8 & 3.39 \\
23451 & 23.8 & 1 & 1.12 & 1.69 & 1.97 & 2.43 & 2.24 & 2.74 \\
23514 & 22.63 & 1 & 1.12 & 1.66 & 1.92 & 1.94 & 1.78 & 3.22 \\
23541 & 23.32 & 1 & 1.14 & 1.66 & 1.93 & 2.45 & 2.28 & 2.71 \\
24135 & 23.25 & 1 & 1.13 & 1.52 & 1.56 & 1.59 & 1.37 & 3.39 \\
24153 & 24.96 & 1 & 1.15 & 1.56 & 1.58 & 2.95 & 2.8 & 3.65 \\
24315 & 23.72 & 1 & 1.15 & 1.57 & 2.05 & 2.07 & 1.9 & 3.49 \\
24351 & 24.66 & 1 & 1.16 & 1.66 & 2.11 & 2.64 & 2.4 & 2.95 \\
24513 & 23.57 & 1 & 1.14 & 1.63 & 2.08 & 2.05 & 1.9 & 2.91 \\
24531 & 23.5 & 1 & 1.14 & 1.59 & 2.09 & 2.52 & 2.42 & 2.89 \\
25134 & 23.42 & 1 & 1.14 & 1.55 & 1.58 & 1.56 & 1.37 & 3.5 \\
25143 & 24.98 & 1 & 1.14 & 1.54 & 1.57 & 3.01 & 2.8 & 3.49 \\
25314 & 22.98 & 1 & 1.15 & 1.55 & 2.06 & 2.08 & 1.91 & 3.52 \\
25341 & 23.52 & 1 & 1.14 & 1.65 & 2.08 & 2.6 & 2.37 & 2.83 \\
25413 & 24.15 & 1 & 1.18 & 1.65 & 2.15 & 2.15 & 1.96 & 3.02 \\
25431 & 24.38 & 1 & 1.13 & 1.64 & 2.11 & 2.62 & 2.45 & 2.92 \\
\hline & & & & & & & &
\end{tabular}

Figure 44: vast-2015-mc1-5d results normalized by splatt (parallel) (3)

$\begin{array}{llccccccc} & \text { qsort } & \text { splatt } & \text { 1-sadilla } & 2 \text {-sadilla } & 3 \text {-sadilla } & 4 \text {-sadilla } & \text { k-sadilla } & \text { radix } \\ 31245 & 2.97 & 1 & 1.07 & 0.15 & 0.16 & 0.16 & 0.12 & 0.9 \\ 31254 & 3.01 & 1 & 1.06 & 0.15 & 0.16 & 0.43 & 0.39 & 0.89 \\ 31425 & 1.84 & 1 & 0.99 & 7.4 \cdot 10^{-2} & 0.17 & 0.17 & 0.15 & 0.38 \\ 31452 & 1.77 & 1 & 0.99 & 7.2 \cdot 10^{-2} & 0.16 & 0.26 & 0.25 & 0.34 \\ 31524 & 1.88 & 1 & 1 & 7.5 \cdot 10^{-2} & 0.17 & 0.17 & 0.15 & 0.37 \\ 31542 & 1.79 & 1 & 1 & 7.4 \cdot 10^{-2} & 0.16 & 0.27 & 0.24 & 0.35 \\ 32145 & 1.35 & 1 & 1 & 9.3 \cdot 10^{-2} & 9.3 \cdot 10^{-2} & 9.2 \cdot 10^{-2} & 8.2 \cdot 10^{-2} & 0.21 \\ 32154 & 1.41 & 1 & 1 & 9.4 \cdot 10^{-2} & 9.3 \cdot 10^{-2} & 0.17 & 0.16 & 0.21 \\ 32415 & 1.31 & 1 & 0.99 & 9.7 \cdot 10^{-2} & 0.11 & 0.12 & 0.11 & 0.2 \\ 32451 & 1.36 & 1 & 1 & 9.8 \cdot 10^{-2} & 0.11 & 0.14 & 0.13 & 0.15 \\ 32514 & 1.32 & 1 & 1 & 9.9 \cdot 10^{-2} & 0.12 & 0.12 & 0.11 & 0.19 \\ 32541 & 1.32 & 1 & 1 & 9.5 \cdot 10^{-2} & 0.11 & 0.14 & 0.13 & 0.16 \\ 34125 & 1.42 & 1 & 0.99 & 6.7 \cdot 10^{-2} & 6.4 \cdot 10^{-2} & 6.4 \cdot 10^{-2} & 5.2 \cdot 10^{-2} & 0.2 \\ 34152 & 1.37 & 1 & 0.99 & 7.1 \cdot 10^{-2} & 6 \cdot 10^{-2} & 0.12 & 0.11 & 0.17 \\ 34215 & 1.43 & 1 & 1 & 9.2 \cdot 10^{-2} & 0.1 & 0.1 & 9.3 \cdot 10^{-2} & 0.18 \\ 34251 & 1.43 & 1 & 0.99 & 8.9 \cdot 10^{-2} & 0.1 & 0.12 & 0.11 & 0.14 \\ 34512 & 1.28 & 1 & 0.99 & 9.2 \cdot 10^{-2} & 7.7 \cdot 10^{-2} & 8 \cdot 10^{-2} & 7 \cdot 10^{-2} & 0.17 \\ 34521 & 1.45 & 1 & 1 & 9.5 \cdot 10^{-2} & 8.8 \cdot 10^{-2} & 0.12 & 0.11 & 0.14 \\ 35124 & 1.41 & 1 & 0.99 & 6.3 \cdot 10^{-2} & 6.2 \cdot 10^{-2} & 6.3 \cdot 10^{-2} & 5.2 \cdot 10^{-2} & 0.19 \\ 35142 & 1.33 & 1 & 1 & 6.6 \cdot 10^{-2} & 6.2 \cdot 10^{-2} & 0.11 & 0.11 & 0.18 \\ 35214 & 1.44 & 1 & 1 & 8.6 \cdot 10^{-2} & 0.1 & 0.1 & 9.4 \cdot 10^{-2} & 0.18 \\ 35241 & 1.45 & 1 & 1 & 8.2 \cdot 10^{-2} & 0.1 & 0.12 & 0.11 & 0.14 \\ 35412 & 1.31 & 1 & 0.99 & 8.2 \cdot 10^{-2} & 7.9 \cdot 10^{-2} & 7.8 \cdot 10^{-2} & 7.1 \cdot 10^{-2} & 0.17 \\ 35421 & 1.41 & 1 & 1 & 8.3 \cdot 10^{-2} & 8.9 \cdot 10^{-2} & 0.12 & 0.11 & 0.13\end{array}$


Figure 45: vast-2015-mc1-5d results normalized by splatt (parallel) (4)

$\begin{array}{lcccccccc} & \text { qsort } & \text { splatt } & \text { 1-sadilla } & \text { 2-sadilla } & \text { 3-sadilla } & \text { 4-sadilla } & \text { k-sadilla } & \text { radix } \\ 41235 & 41.46 & 1 & 1.12 & 1.06 & 1.06 & 1.06 & 0.79 & 5.83 \\ 41253 & 41.01 & 1 & 1.13 & 1.08 & 1.08 & 3.04 & 2.71 & 5.98 \\ 41325 & 40.9 & 1 & 1.14 & 1.03 & 2.4 & 2.54 & 2.16 & 5.8 \\ 41352 & 36.31 & 1 & 1.12 & 0.95 & 2.19 & 3.78 & 3.46 & 4.76 \\ 41523 & 36.89 & 1 & 1.11 & 0.94 & 2.59 & 2.54 & 2.33 & 4.85 \\ 41532 & 36.84 & 1 & 1.12 & 0.96 & 2.55 & 3.76 & 3.47 & 4.83 \\ 42135 & 25.27 & 1 & 1.05 & 1.36 & 1.32 & 1.33 & 1.19 & 3.05 \\ 42153 & 24.67 & 1 & 1.06 & 1.34 & 1.39 & 2.6 & 2.42 & 3.13 \\ 42315 & 25.02 & 1 & 1.08 & 1.39 & 1.79 & 1.79 & 1.65 & 3.16 \\ 42351 & 24.31 & 1 & 1.08 & 1.48 & 1.85 & 2.18 & 2.04 & 2.5 \\ 42513 & 25.46 & 1 & 1.07 & 1.46 & 1.85 & 1.86 & 1.63 & 2.64 \\ 42531 & 24.09 & 1 & 1.05 & 1.42 & 1.72 & 2.21 & 2.04 & 2.51 \\ 43125 & 30.43 & 1 & 1.06 & 1.5 & 1.34 & 1.35 & 1.15 & 4.39 \\ 43152 & 28.6 & 1 & 1.06 & 1.46 & 1.29 & 2.57 & 2.34 & 3.74 \\ 43215 & 22.53 & 1 & 1.06 & 1.47 & 1.61 & 1.63 & 1.48 & 2.84 \\ 43251 & 23.44 & 1 & 1.05 & 1.47 & 1.67 & 2.03 & 1.84 & 2.25 \\ 43512 & 21.04 & 1 & 1.04 & 1.49 & 1.26 & 1.26 & 1.09 & 2.78 \\ 43521 & 23.26 & 1 & 1.07 & 1.53 & 1.46 & 1.91 & 1.8 & 2.22 \\ 45123 & 22.31 & 1 & 1.06 & 0.93 & 0.97 & 0.97 & 0.83 & 3.06 \\ 45132 & 22.91 & 1 & 1.05 & 0.96 & 0.99 & 1.72 & 1.54 & 3.04 \\ 45213 & 24.26 & 1 & 1.07 & 1.12 & 1.63 & 1.63 & 1.54 & 2.35 \\ 45231 & 23.4 & 1 & 1.05 & 1.12 & 1.63 & 2.07 & 1.92 & 2.29 \\ 45312 & 21.81 & 1 & 1.04 & 0.98 & 1.32 & 1.34 & 1.18 & 2.91 \\ 45321 & 22.46 & 1 & 1.06 & 1.15 & 1.44 & 1.94 & 1.8 & 2.22\end{array}$

Figure 46: vast-2015-mc1-5d results normalized by splatt (parallel) (5)

\begin{tabular}{|c|c|c|c|c|c|c|c|c|}
\hline & qsort & splatt & 1-sadilla & 2-sadilla & 3-sadilla & 4-sadilla & k-sadilla & radix \\
\hline 51234 & 42.16 & 1 & 1.17 & 1.07 & 1.07 & 1.07 & 0.78 & 5.95 \\
\hline 51243 & 41.33 & 1 & 1.15 & 1.08 & 1.07 & 3 & 2.65 & 6 \\
\hline 51324 & 41.2 & 1 & 1.13 & 1.05 & 2.41 & 2.41 & 2.12 & 5.87 \\
\hline 51342 & 37.04 & 1 & 1.1 & 1 & 2.19 & 3.8 & 3.57 & 4.84 \\
\hline 51423 & 37.65 & 1 & 1.09 & 0.99 & 2.59 & 2.63 & 2.31 & 4.84 \\
\hline 51432 & 38.03 & 1 & 1.14 & 1.01 & 2.65 & 4 & 3.59 & 4.97 \\
\hline 52134 & 25.57 & 1 & 1.07 & 1.33 & 1.35 & 1.35 & 1.2 & 3.06 \\
\hline 52143 & 24.29 & 1 & 1.06 & 1.32 & 1.34 & 2.52 & 2.47 & 3.01 \\
\hline 52314 & 24.48 & 1 & 1.05 & 1.32 & 1.75 & 1.72 & 1.64 & 3.01 \\
\hline 52341 & 24.32 & 1 & 1.06 & 1.43 & 1.8 & 2.2 & 2.09 & 2.5 \\
\hline 52413 & 25.35 & 1 & 1.08 & 1.42 & 1.8 & 1.81 & 1.65 & 2.58 \\
\hline 52431 & 24.51 & 1 & 1.06 & 1.42 & 1.79 & 2.21 & 2.09 & 2.48 \\
\hline 53124 & 30.37 & 1 & 1.05 & 1.46 & 1.34 & 1.38 & 1.13 & 4.28 \\
\hline 53142 & 28.81 & 1 & 1.06 & 1.46 & 1.31 & 2.61 & 2.36 & 3.74 \\
\hline 53214 & 23.36 & 1 & 1.05 & 1.49 & 1.62 & 1.65 & 1.51 & 2.83 \\
\hline 53241 & 23.36 & 1 & 1.04 & 1.45 & 1.63 & 1.97 & 1.87 & 2.26 \\
\hline 53412 & 21.75 & 1 & 1.06 & 1.55 & 1.33 & 1.31 & 1.15 & 2.78 \\
\hline 53421 & 22.62 & 1 & 1.05 & 1.46 & 1.38 & 1.84 & 1.7 & 2.1 \\
\hline 54123 & 23 & 1 & 1.06 & 0.95 & 0.99 & 0.98 & 0.83 & 3.07 \\
\hline 54132 & 22.55 & 1 & 1.06 & 0.95 & 0.98 & 1.7 & 1.54 & 2.97 \\
\hline 54213 & 23.99 & 1 & 1.06 & 1.09 & 1.65 & 1.61 & 1.47 & 2.29 \\
\hline 54231 & 23.14 & 1 & 1.06 & 1.13 & 1.66 & 2.02 & 1.86 & 2.3 \\
\hline 54312 & 22.17 & 1 & 1.05 & 0.96 & 1.34 & 1.36 & 1.17 & 2.94 \\
\hline 54321 & 23.06 & 1 & 1.05 & 1.13 & 1.49 & 1.97 & 1.84 & 2.22 \\
\hline
\end{tabular}

